\title{
Algunas nociones de equilibrio en sistemas estocásticos
}

\author{
Tesis que presenta: \\ Lizeth Marianita Hernández Hernández \\ para obtener el grado de \\ Maestro en Ciencias \\ (Matemáticas Aplicadas e Industriales) \\ Posgrado en Matemáticas \\ Departamento de Matemáticas \\ Universidad Autónoma Metropolitana \\ Iztapalapa
}

Asesor: Dr. Julio César García Corte.

Marzo, 2012. 


\section{Agradecimientos}

Agradezco a Dios por llenar mi vida de dicha y bendiciones.

Dedico esta tesis a mis Padres quienes siempre me han apoyado y amado.

Para mis hermanos que siempre me brindan apoyo, fuerza y amor.

Gracias a mi asesor Julio César García Corte por su guía, paciencia, visión y tiempo para realizar este proyecto.

Gracias a Roberto Quezada Batalla y a Jorge León Vazquez por la revisión y su función como sinodales.

Gracias a los Profesores y amigos que ayudaron al desarrollo de la tesis. Gracias al apoyo de CONACyT con el número de beca 230241. 


\section{Introducción}

Los procesos estocásticos dependen de leyes causales y probabilísticas; están sometidos al azar y son objetos de análisis estadístico. Se centran en el estudio y modelización de sistemas que evolucionan en el espacio y en el tiempo con leyes de carácter aleatorio.

Los procesos estocásticos se clasifican de diversas formas; por ejemplo, atendiendo al carácter del espacio de estados ó del espacio paramétrico, se tienen los procesos discretos y continuos. Otra clasificación se basa en las relaciones de dependencia existentes entre las variables del proceso y en este sentido tenemos por ejemplo: Procesos con incrementos independientes; Procesos de Markov; Martingalas; Procesos estacionarios.

En el capítulo I de este trabajo se consideran algunos conceptos y resultados preliminares para los siguientes capítulos. Así como también una breve introducción sobre teoría de semigrupos.

Los procesos estocásticos que se consideran en el capítulo II son los procesos estacionarios, tanto en sentido estricto como en sentido amplio. La estacionariedad es tomada como una noción de equilibrio y suele ser la situación a considerar en gran parte de los supuestos de la termodinámica [17].

En el capítulo III de este trabajo se consideran los procesos de Markov en tiempo discreto (llamadas cadenas de Markov) y en tiempo continuo, donde se presenta otra noción de equilibrio llamada balance detallado debida a Boltzmann, la cual es equivalente a la noción de irreversibilidad de una cadena de Markov dada por Kolmogorov [13], que establece que la probabilidad de visitar los estados $x_{1}, x_{2}, x_{3}, \ldots x_{n}, x_{1}$ en ese orden, es la misma que visitarlos en sentido inverso, es decir, $x_{1}, x_{n}, \ldots, x_{2}, x_{1}$ y es el estado de referencia en termodinámica de procesos irreversibles [13]. Es importante mencionar que un proceso de Markov que satisface 
la condición de balance detallado es estacionario en sentido estricto.

Por otro lado, explicar los fenómenos irreversibles en sistemas macroscópicos a partir de la evolución reversible de sus componentes microscópicas, dando predicciones cuantitativas, es el problema fundamental de la mecánica estadística [18]. Ésta consiste de dos partes diferentes: la mecánica estadística de sistemas en equilibrio y aquella de sistemas fuera de equilibrio. La relación entre los estados macroscópicos y microscópicos en la mecánica estadística está dada por la fórmula de entropía dada por Boltzmann, donde también se utiliza la condición de balance detallado [13].

En el último capítulo de este trabajo se consideran los sistemas clásicos lattice que se pueden considerar como procesos estocásticos con parámetro de tiempo $T=\mathbb{Z}^{N}$. En estos sistemas el equilibrio es maximizar la ecuación del principio variacional [8]. En este sentido, los estados de equilibrio son estados de Gibbs, los cuales son medidas de probabilidad específicas, el equilibrio en la lattice se mantiene a través del balance detallado [13]. Se sigue a David Ruelle [8] en este capítulo el cual da un formalismo en sistemas clásicos lattice.

Como bien se menciona en [13], un sistema que no está en equilibrio es un sistema estacionario abierto con tasa de producción de entropía positiva, es decir, intercambia sustancias y energía con su medio ambiente. Lo que nos impone la condición de balance detallado es que, en media, el sistema pasa tantas veces del estado $x$ al estado $y$ como del estado $y$ al estado $x$, lo cual tiene una ventaja física. La mayoria de los sistemas que interesa simular siguen las leyes de la mécanica clásica o cuántica, las cuales son invariantes bajo traslaciones del tiempo. Con la condición de balance detallado, una simulación también lo es, siendo así una representación más adecuada de lo que se observa en la naturaleza.

Así pues tenemos tres nociones de equilibrio que podrían pensarse ajenas, pero que en realidad están relacionadas de la siguiente forma:

\section{Balance Detallado $\Leftrightarrow$ Equilibrio en el sentido de Gibbs. Balance Detallado $\Rightarrow$ Estacionariedad Estricta.}

A lo largo del trabajo se exponen algunos ejemplos de sistemas en equilibrio y de no equilibrio. 


\section{Resumen}

Se presentan algunas nociones de equilibrio en sistemas estocásticos y se estudian algunas relaciones entre ellas. Mostramos algunos ejemplos con las nociones de equilibrio consideradas de los cuales algunos están en equilibrio y otros fuera de equilibrio. 


\section{Índice general}

Introducción $\quad$ V

$\begin{array}{lll}\text { Resumen } & \text { VII }\end{array}$

$\begin{array}{ll}\text { 1. Preliminares } & 1\end{array}$

1.1. Generalidades sobre Procesos Estocásticos . . . . . . . . . . . . . . . . 1

1.2. Teoría elemental de semigrupos . . . . . . . . . . . . . . . 5

2. Procesos Estocásticos Estacionarios $\quad 9$

2.1. Procesos Estocásticos de Segundo Orden . . . . . . . . . . . . . . . . . 9

2.1.1. Construcción de un proceso estacionario en sentido amplio . . . . . . . . 13

2.2. Procesos Estacionarios en Sentido Estricto . . . . . . . . . . . . . . 15

2.3. Teorema Ergódico . . . . . . . . . . . . . . . . . . . . 17

IX 
3.1. Cadenas de Markov en tiempo discreto. . . . . . . . . . . . . . . . . . 21

3.1.1. Cadena de Markov con espacio de estados finito . . . . . . . . . . . . . 27

3.1.2. Cadena de Markov con espacio de estados infinito numerable . . . . . . 30

3.1.3. Versión del Teorema Ergódico para cadenas de Markov . . . . . . . . . . 32

3.2. Proceso de Markov en tiempo continuo . . . . . . . . . . . . . . 33

3.2.1. Construcción de un proceso de Markov . . . . . . . . . . . . . 33

3.2.2. Semigrupo de Markov . . . . . . . . . . . . . . . . . 35

3.3. Cadena de Markov encajada de un proceso de Markov de Saltos. . . . . . . . . 42

3.3.1. Ecuación Integral hacia atrás de Kolmogorov . . . . . . . . . . . . . . 50

3.3.2. Proceso de Poisson . . . . . . . . . . . . . . . 58

3.3.3. Proceso de Poisson Compuesto . . . . . . . . . . . . . . . . . 59

3.4. Balance Detallado . . . . . . . . . . . . . . . . . . . 61

3.4.1. Proceso con espacio de estados finito . . . . . . . . . . . . . 62

3.4.2. Proceso con espacio de estados infinitos numerable. . . . . . . . . . 65 
4.1. Sistemas Lattice Finitos . . . . . . . . . . . . . . . . . . 70

4.2. Sistemas Lattice Infinitos . . . . . . . . . . . . . . . . 72 


\section{Capítulo 1}

\section{Preliminares}

\subsection{Generalidades sobre Procesos Estocásticos}

Sea $(S, \mathfrak{S})$ un espacio medible y $T$ cualquier subconjunto de $\mathbb{N}, \mathbb{Z}, \mathbb{R}, \mathbb{R}_{+}$ó $\mathbb{Z}^{N}$, donde $N \in \mathbb{N}=\{0,1,2, \ldots\}$. Sea $\mathfrak{S}$ una $\sigma$-álgebra sobre $S$ y $(\Omega, \mathfrak{F}, P)$ un espacio de probabilidad.

Definición 1. Sea $(S, \mathfrak{S})$ un espacio medible, un núcleo estocástico es una función $p$ : $S \times \mathfrak{S} \rightarrow[0,1]$ tal que

1. Para todo e $\in S$ fijo, $A \longrightarrow p(e, A), A \in \mathfrak{S}$, es una medida de probabilidad.

2. Para todo $A \in \mathfrak{S}$ fijo, $e \longrightarrow p(e, A)$ es una función medible.

Definición 2. Sea $(\Omega, \mathfrak{F}, P)$ espacio de probabilidad y $(S, \mathfrak{S})$ espacio medible. Sea $\mathfrak{Y} \subset \mathfrak{F}$ una sub- $\sigma$-álgebra de $\mathfrak{F}$. Sea $X$ variable aleatoria tal que $X: \Omega \longrightarrow S$. Decimos que $\mu$ tal que $\mu: \Omega \times \mathfrak{S} \longrightarrow[0,1]$ es una distribución condicional regular de $X$ dada $\mathfrak{Y}$ si cumple con,

1. Existe $\Omega_{0} \in \mathfrak{F}, P\left(\Omega_{0}\right)=1$ tal que para todo $\omega \in \Omega_{0}$ fijo, la función $B \longmapsto \mu(\omega, B)$ es medida de probabilidad en $(S, \mathfrak{S})$.

2. Para toda $B \in \mathfrak{S}$ la función $\omega \longmapsto \mu(\omega, B)$ es una versión de $P[X \in B \mid \mathfrak{Y}]$. 
Definición 3. Sea $(S, \mathfrak{S})$ espacio medible. Una función de transición es una función $p:[0, \infty) \times S \times \mathfrak{S} \rightarrow[0,1]$ tal que

1. $p(t, \cdot, \cdot)$ es núcleo estocástico para toda $t \in[0, \infty)$.

2. Satisface la ecuación de Champan-Kolmogorov

$$
p(t+s, x, A)=\int_{S} p(t, x, d y) p(s, y, A) .
$$

3. $p(0, x, A)=\delta_{x}(A)$.

4. $\int_{S} p(t, x, d y) f(y) \longrightarrow f(x)$ cuando $t \rightarrow 0$.

Definición 4. Sea $(S, \mathfrak{S})$ un espacio medible. Sea $p: S \times \mathfrak{S} \longrightarrow[0,1]$ un núcleo estocástico. Sea $\mu: S \longrightarrow[0, \infty]$ una medida, decimos que $\mu$ es invariante respecto a $p$ si

$$
\int_{S} \mu(d e) p(e, A)=\mu(A)
$$

Además si $\mu$ es medida de probabilidad diremos que $\mu$ es distribución invariante.

Sea $(S, \mathfrak{S})$ un espacio medible y sean $\left\{n_{1}, n_{2}, \ldots, n_{k}\right\} \subset T$ con $k \in \mathbb{N}$. La proyección $k$ dimensional es la función $\Pi_{n_{1}, \ldots, n_{k}}: S^{T} \longrightarrow S^{\left\{n_{1}, n_{2}, \ldots, n_{k}\right\}}$ con $S^{\left\{n_{1}, n_{2}, \ldots, n_{k}\right\}}=S \times \ldots \times S$ y $\Pi_{n_{1}, \ldots, n_{k}}\left(a_{0}, a_{1}, \ldots\right)=\left(a_{n_{1}}, a_{n_{2}}, \ldots, a_{n_{k}}\right)$.

Identificaremos al conjunto $S^{T}$ como el conjunto de las funciones continuas tales que $S^{T}=\{f: T \longrightarrow S\}$. Por lo tanto para $F=\left\{t_{1}, t_{2}, \ldots, t_{k}\right\} \subset T$ finito, $S^{F}=\{f: F \longrightarrow S\}$.

Una familia $\mathbf{F}=\left\{\Pi_{n_{1} \ldots n_{k}}: k \in \mathbb{N}, k \geq 1\right.$ y $\left.n_{i} \in T\right\}$ es una familia de proyecciones $k$-dimensionales con $k$ fijo.

Definición 5. Sea $\left\{X_{t}\right\}_{t \in T}$ un proceso estocástico. Sea $F \subset T$ un subconjunto finito tal que $F=\left\{t_{1}, t_{2}, \ldots, t_{n}\right\}$ con $n \in \mathbb{N}$. La distribución finito dimensional del proceso $\left\{X_{t}\right\}_{t \in T}$ sobre $F$ es la distribución del vector aleatorio $\left(X_{t_{1}}, X_{t_{2}}, \ldots, X_{t_{n}}\right)$.

Sea $F \subset T$ subconjunto finito. La $\sigma$-álgebra producto $\mathfrak{S}^{F}$ de $S^{F}$ es la $\sigma$-álgebra generada por conjuntos de la forma $A_{1} \times A_{2} \times \ldots \times A_{k}$ donde $A_{i} \in \mathfrak{S}$ con $i=1, \ldots, k$. 
La $\sigma$-álgebra cilíndrica $\mathcal{C}$ es la menor $\sigma$-álgebra que hace medibles a las proyecciones finito dimensionales; es $\operatorname{decir} \mathcal{C}=\left\{\Pi_{n_{1} \ldots n_{k}}^{-1}(A): k \in T, n_{1}, \ldots, n_{k} \in T, A \in \mathfrak{S}^{F}\right\}$.

Definición 6. Sea $(S, \mathfrak{S})$ un espacio medible y $F \subset T$ con $F$ finito. Sea $P_{F}: \mathfrak{S}^{F} \longrightarrow[0,1]$ una medida de probabilidad en $\mathfrak{S}^{F}$. Decimos que la familia $\left\{P_{F}: F \subset T, F\right.$ finito $\}$ es un sistema proyectivo si $F \subset G \subset T$ con $G$ finito, entonces $P_{F}=\left(P_{G}\right)_{\Pi_{F}^{G}}$, es decir, la medida de probabilidad en $F$ es la proyección de $F$ en $G$ de $P_{G}$.

Definición 7. Sea $(S, \mathfrak{S})$ espacio medible. Supongamos que $\left\{P_{F}: F \subset T, F\right.$ finito $\}$ es un sistema proyectivo. Una medida de probabilidad

$$
P: \mathcal{C} \longrightarrow[0,1]
$$

se dice que es un límite proyectivo del sistema proyectivo $\left\{P_{F}: F \subset T, F\right.$ finito $\}$ si $F \subset T$, $F$ finito y $P_{F}=P_{\Pi_{F}}$. Además este límite es único.

Observemos que $P_{F}$ es la medida de probabilidad en $F$ y $P_{\Pi_{F}}$ es la distribución de probabilidad de la proyección $\Pi_{F}$.

Teorema 1. (Teorema de Consistencia de Kolmogorov). Sea $S$ un espacio métrico completo y separable. Sea $\mathfrak{S}=\mathfrak{B}(S)$ la $\sigma$-álgebra de Borel en $S$. Sea $T$ un conjunto cualesquiera para cada $F \subset T$ finito. Sea $P_{F}: \mathfrak{S}^{F} \longrightarrow[0,1]$ una medida de probabilidad. Supóngase que $\left\{P_{F}: F \subset T, F\right.$ finito $\}$ es un sistema proyectivo. Entonces

$$
\left\{P_{F}: F \subset T, F \text { finito }\right\}
$$

tiene límite proyectivo único.

Demostración. Ver [4], pp. 25-28.

Teorema 2. (La construcción de Kolmogorov). Sea $S$ un espacio métrico completo y separable y para cada $F \subset T$ con $F$ finito, sea $P_{F}$ una probabilidad en $S^{F}$ tal que el sistema

$$
\left\{S^{F}, \mathfrak{S}^{F}, P_{F}, \Pi_{F}^{L}\right\}_{F \subset L \subset T, F, L} \text { finitos }
$$

sea proyectivo. Entonces el sistema 1.1 tiene límite proyectivo $P$, único. En particular el sistema canónico $\left(S^{F}, \mathfrak{S}^{F}, P,\left\{\Pi_{t}\right\}_{t \in T}\right)$ satisface que $P \Pi_{F}^{-1}=P_{F}$, para todo $F \subset T$ y $F$ finito, es decir, el proceso $\left\{\Pi_{t}\right\}_{t \in T}$ tiene cono distribuciones finito dimensionales a la familia $\left\{P_{F}\right\}_{F \subset T, F}$ finito.

Demostración. Para cada $F \subset T, F$ finito, el espacio $\left(S^{T}, \mathfrak{S}^{F}\right)$ satisface las hipótesis del Teorema de consistencia de Kolmogorov y sea $P$ el límite proyectivo dado por el Teorema de consistencia de Kolmogorov. Entonces el proceso $\left(S^{T}, \mathfrak{S}^{T}, P,\left\{\pi_{t}\right\}_{t \in T}\right)$ satisface las condiciones deseadas. 
Tenemos las siguientes nociones de equivalencia entre dos procesos estocásticos.

Definición 8. Sean $(\Omega, \mathfrak{F}, P)$ y $\left(\Omega^{\prime}, \mathfrak{F}^{\prime}, P\right)$. Sean $\left\{X_{t}\right\}_{t \in T}$ y $\left\{Y_{t}\right\}_{t \in T}$ procesos estocásticos

i) Si $X_{t}: \Omega \rightarrow S$ y $Y_{t}: \Omega^{\prime} \rightarrow S$ decimos que los procesos son equivalentes si para toda $n \in \mathbb{N}, t_{1}<t_{2}<\ldots<t_{n}$ el vector aleatorio $\left(X_{t_{1}}, X_{t_{2}}, \ldots, X_{t_{n}}\right)$ tiene la misma distribución que $\left(Y_{t_{1}}, Y_{t_{2}}, \ldots, Y_{t_{n}}\right)$.

ii) Si $X_{t}, Y_{t}: \Omega \rightarrow S$, decimos que un proceso es modificación del otro si $\forall t \in T$, $P\left(X_{t}=Y_{t}\right)=1$.

iii) Si $X_{t}, Y_{t}: \Omega \rightarrow S$, decimos que los procesos son indistinguibles si existe $A \in \mathfrak{F}$, $P(A)=1$ tal que $A \subset\left\{\omega \in \Omega: X_{t}(\omega)=Y_{t}(\omega) \quad \forall t \in T\right\}$

Observemos que dados dos procesos $\left\{X_{t}\right\}_{t \in T}$ y $\left\{Y_{t}\right\}_{t \in T}$ si uno es modificación del otro entonces son equivalentes. Si los procesos son indistinguibles entonces uno es modificación de otro. Se sabe que las afirmaciones recíprocas son falsas. Lo mostramos con el siguiente ejemplo en donde dos procesos serán equivalentes, aún más uno modificación de otro pero, en general, no serán indistiguibles. Esto se debe a la no medibilidad de algunos conjuntos de interés.

Ejemplo 1. Si $S=\{0,1\}, T=[0,1], \mathfrak{B}=2^{S}$ y $A=\{0\}$. Entonces $A \notin \mathfrak{B}^{T}$.

Demostración. Consideremos el espacio de probabilidad $(\Omega, \mathcal{L}, \lambda)$ donde $\Omega=[0,1], \mathcal{L}=$ $\sigma$-álgebra de los Lebesgue medibles en $[0,1]$ y $\lambda=$ la medida de Lebesgue. Sean $\Phi, \Psi: \Omega \rightarrow S^{T}$ tales que, dado $\omega \in \Omega$

$$
\begin{gathered}
\Phi(\omega): T \rightarrow S, \quad \Phi(\omega, t)=0 \quad \forall \quad(\omega, t) \in \Omega \times T \\
\Psi(\omega): T \rightarrow S, \quad \Psi(\omega, t)= \begin{cases}0, & \forall \in T \backslash\{\omega\}=1_{\{\omega\}}(t) \\
1, & \text { si } t=\omega\end{cases}
\end{gathered}
$$

Observemos que $\Phi(\omega)$ es simplemente la función constante cero, es decir, $0=(0,0, \ldots) \in S^{T}$. Por tanto $\Phi(\Omega)=\{0\}=A$. Sin embargo, $\Psi(\Omega) \bigcap A=\emptyset$. Sea $\mathcal{C}$ la $\sigma$-álgebra cílindrica.

Ahora usemos el hecho de que $A \in \mathcal{C}\left(S^{T}\right)$ si y sólo si existe $\left\{t_{1} t_{2} \ldots\right\} \subset T$ y existe $B \in$ $\mathcal{C}\left(S^{\left\{t_{1} t_{2} \ldots\right\}}\right)$ tal que $A=\pi_{\left\{t_{1} t_{2} \ldots\right\}}^{-1}(B)$. Por tanto, si $\{0\} \in \mathcal{C}\left(S^{T}\right)$ entonces existe $\left\{t_{1} t_{2} \ldots\right\} \subset T$, existe $B \in \mathcal{C}\left(S^{\left\{t_{1} t_{2} \ldots\right\}}\right)$ tal que $\{0\}=\pi_{\left\{t_{1} t_{2} \ldots\right\}}^{-1}(B)$, es decir, Si $y \in T \backslash\left\{t_{1} t_{2} \ldots\right\}$ y $f=\mathbf{1}_{\{y\}}$, $f\left(t_{i}\right)=0$ se tiene que

$$
\pi_{\left\{t_{1} t_{2} \ldots\right\}} f=(0,0, \ldots) \in B
$$


por lo tanto $f \in \pi_{\left\{t_{1} t_{2} \ldots\right\}}^{-1}(B)=\{0\}$ lo cual es una contradicción a que $A \in \mathcal{C}\left(S^{T}\right)$.

Para todo $t$ fijo, tenemos que

$$
\{\omega \in \Omega: \Psi(\omega, t)=\Phi(\omega, t)\}=\left\{\omega \in \Omega: \mathbf{1}_{\{\omega\}}(t)=0\right\}=[0,1] \backslash\{t\}
$$

por lo tanto $\Psi$ y $\Phi$ son equivalentes. $Y$ además

$$
\{\omega \in \Omega: \Psi(\omega, t)=\Phi(\omega, t) \text { para toda } t \geq 0\}=\left\{\omega \in \Omega: \mathbf{1}_{\{\omega\}}(t)=0 \text { para toda } t \geq 0\right\}=\emptyset
$$

es decir, $\Psi$ es modificación de $\Phi$.

Sea $C \in \mathcal{C}$ con $C$ un cílindro finito, entonces

$$
\Psi^{-1}(C)=\left\{\omega \in \Omega: \Psi\left(\omega, t_{1}\right) \in B_{1}, \ldots, \Psi\left(\omega, t_{n}\right) \in B_{n}\right\} .
$$

$y \Phi^{-1}(C)=\Omega$ si $0 \in \bigcap_{i=1}^{n} B_{i} y \emptyset$ en otro caso. Por tanto $\Psi^{-1}(C)$ difiere de $\Phi^{-1}(C)$ en un conjunto de medida cero. Por ser completa la medida de lebesgue $\Psi^{-1}(C) \in \mathcal{L}$ para todo $C \in \mathcal{C}$. Por lo tanto $\Psi$ es medible de $(\Omega, \mathcal{L})$ en $\left(S^{T}, \mathfrak{B}^{T}\right)$.

Sean $\boldsymbol{P}=\lambda \circ \Phi^{-1}, \boldsymbol{Q}=\lambda \circ \Psi^{-1}$ distribuciones de $\Phi$ y $\Psi$ en $\left(S^{T}, \mathfrak{B}^{T}\right)$, así

$$
\begin{aligned}
\boldsymbol{P}(C) & =\lambda\left(\left\{\omega \in \Omega: \Phi\left(\omega, t_{1}\right) \in B_{1}, \ldots, \Phi\left(\omega, t_{n}\right) \in B_{n}\right\}\right) \\
& =\lambda\left(\left\{\omega \in \Omega: \Psi\left(\omega, t_{1}\right) \in B_{1}, \ldots, \Psi\left(\omega, t_{n}\right) \in B_{n}\right\}\right) \\
& =\boldsymbol{Q}(C) .
\end{aligned}
$$

Entonces $\boldsymbol{P}$ y $\boldsymbol{Q}$ coinciden en $\mathcal{C}$ y por tanto en todo $\mathfrak{B}^{T}$. Ahora supongamos que $A \in \mathfrak{B}^{T}$, entonces

$$
\begin{gathered}
\boldsymbol{P}(A)=\lambda\left(\Phi^{-1}(A)\right)=\lambda(\Omega)=0 \\
\boldsymbol{Q}(A)=\lambda\left(\Psi^{-1}(A)\right)=\lambda(\emptyset)=1
\end{gathered}
$$

es decir, $\boldsymbol{P}$ y $\boldsymbol{Q}$ no coinciden en $\mathfrak{B}^{T}$ lo cual es una contradicción, por tanto $A \notin \mathfrak{B}^{T}$.

\subsection{Teoría elemental de semigrupos}

En las matemáticas existe una gran conexión entre lo que son los procesos estocásticos y los semigrupos de operadores. En la actualidad estas conexiones han permitido el desarrollo de 
métodos estocásticos para el estudio de problemas de aplicación. En esta sección se presenta una breve introducción de semigrupos y la conexión con los procesos estocásticos.

Definición 9. Sea $V$ un espacio de Banach, con norma $\|\cdot\|$. Un semigrupo fuertemente continuo sobre $V$, es una familia de transformaciones lineales y acotadas $\left\{T_{t}\right\}_{t \geq 0}, T_{t}: V \longrightarrow$ $V$ con las siguientes propiedades:

1. $T_{t} T_{s}=T_{s+t}=T_{s} T_{t}$

2. $T_{0} f=f$ para toda $f \in V$

3. $\lim _{t \rightarrow 0^{+}}\left\|T_{t} f-f\right\|=0$ para toda $f \in V$

Si $\left\{T_{t}\right\}_{t \geq 0}$ cumple con las condiciones anteriores, diremos simplemente que $\left\{T_{t}\right\}_{t \geq 0}$ es un semigrupo.

Teorema 3. Sea $\left\{T_{t}\right\}_{t \geq 0}$ un semigrupo y $V$ un espacio de Banach. Entonces para todo $t \geq 0$ fijo, $\operatorname{lím}_{h \rightarrow 0}\left\|T_{t+h} f-T_{t} f\right\|=0$ para toda $f \in V$.

Demostración. Tenemos que para $t \geq 0, f \in V$ fijos con $g=T_{t} f \in V$

$$
\begin{aligned}
\left\|T_{t+h} f-T_{t} f\right\| & =\left\|T_{h} T_{t} f-T_{t} f\right\| \\
& =\left\|T_{h} g-g\right\|
\end{aligned}
$$

pero $\left\|T_{h} g-g\right\| \rightarrow 0$ cuando $h \rightarrow 0$, por lo tanto

$$
\left\|T_{t+h} f-T_{t} f\right\| \longrightarrow_{h \rightarrow 0} 0
$$

Definición 10. Sea $\left\{T_{t}\right\}_{t \geq 0}$ un semigrupo en $V$. Sea $D \subset V$, definido como

$$
D=\left\{f \in V: \lim _{t \rightarrow 0} \frac{T_{t} f-f}{t} \text { existe en } V\right\} .
$$

Sea $L: D \rightarrow V$ y $L f=\lim _{t \rightarrow 0} \frac{T_{t} f-f}{t}$. Al operador L se le llama el Generador infinitesimal del semigrupo $\left\{T_{t}\right\}_{t \geq 0}$

Corolario 1. El conjunto $D$ es subespacio vectorial de $V$ y $L$ es un operador lineal. 
Demostración. Tenemos que $D=\left\{f \in V: \operatorname{lím}_{t \rightarrow 0} \frac{T_{t} f-f}{t}\right.$ existe $\}$, entonces $D \subseteq V$ y $D \neq \emptyset$ ya que $0 \in D$. Sean $f, g \in D$, entonces

$$
\begin{aligned}
\lim _{t \rightarrow 0} \frac{T_{t}(f+g)-(f+g)}{t} & =\lim _{t \rightarrow 0} \frac{T_{t} f+T_{t} g-f-g}{t} \\
& =\lim _{t \rightarrow 0} \frac{T_{t} f-f}{t}+\lim _{t \rightarrow 0} \frac{T_{t} g-g}{t}
\end{aligned}
$$

donde tales límites existen, por tanto $f+g \in D$. Si además $a \in \mathbb{R}$, entonces

$$
\begin{aligned}
\lim _{t \rightarrow 0} \frac{T_{t}(a f)-(a f)}{t} & =\lim _{t \rightarrow 0} \frac{a\left(T_{t} f-f\right)}{t} \\
& =a \lim _{t \rightarrow 0} \frac{T_{t} f-f}{t}
\end{aligned}
$$

así af $\in D$ y $D$ es subespacio vectorial de $V$. Además con el mismo argumento, es claro que $L$ es un operador lineal.

Proposición 1. Sea $L$ el generador infinitesimal del semigrupo $\left\{T_{t}\right\}_{t \geq 0}$ con $D$ su dominio. Si $f \in D$ entonces $T_{t} f \in D$ y $L T_{t} f=T_{t} L f=\frac{d}{d t} T_{t} f$.

Demostración. Ver [3], pp. 139 - 140.

Definición 11. Decimos que el semigrupo es uniformemente continuo si

$$
\sup _{\|f\| \leq 1}\left\|T_{t} f-f\right\| \longrightarrow_{t \rightarrow 0} 0
$$

Para tales semigrupos tenemos el siguiente teorema.

Teorema 4. a) Si $\left\{T_{t}\right\}_{t \geq 0}$ es uniformemente continuo entonces es fuertemente continuo.

b) Si $\left\{T_{t}\right\}_{t \geq 0}$ es un semigrupo fuertemente continuo que actúa sobre $V$, entonces $D$ es denso en $V$.

c) $\left\{T_{t}\right\}_{t \geq 0}$ es uniformemente continuo si y sólo si $D=V$ y $L$ es continuo.

d) Si $V$ es de dimensión finita entonces todo semigrupo es uniformemente continuo.

Demostración. Ver [4], pp. $449-452$. 
Ejemplo 2. Supongamos que $L$ es un operador tal que $D=V$ y $L$ es continuo y acotado. Sea $L^{n}=L \cdots L$ y sea $\sum_{k=0}^{n} \frac{(t L)^{k}}{k !}: V \rightarrow V$. Si $f \in V$, para $t \geq 0$

$$
\left\|\sum_{k=0}^{n} \frac{(t L)^{k}}{k !} f\right\| \leq\left\{\sum_{k=m+1}^{n} \frac{\|t L\|^{k}}{k !}\right\}\|f\| \longrightarrow_{n, m \rightarrow 0} 0
$$

entonces $\left\{\sum_{k=0}^{n} \frac{(t L)^{k}}{k !}\right\}_{n=1}^{\infty}$ es sucesión de Cauchy en $V$. Tomando el límite se obtiene

$$
e^{t L} f:=\lim _{n \rightarrow \infty} \sum_{k=0}^{n} \frac{(t L)^{k}}{k !} f
$$

al operador $T_{t}=e^{t L}: V \rightarrow V$ se le llama el operador exponencial. Como podemos observar el operador exponencial es un semigrupo y para toda $f \in V$

$$
\frac{d}{d t} e^{t L} f=L e^{t L} f
$$

Es importante mencionar que para todo semigrupo uniformemente continuo el generador infinitesimal y el semigrupo de éste es de la forma del ejemplo anterior. Para una prueba ver $[3]$. 


\section{Capítulo 2}

\section{Procesos Estocásticos Estacionarios}

Consideramos en este capítulo la primer noción de equilibrio de este trabajo para procesos estocásticos. Se considera que un proceso estocástico estacionario está en equilibrio. Algunos resultados y ejemplos fueron tomados de [3].

Definición 12. Un proceso estocástico con $T=\mathbb{N}, \mathbb{Z}, \mathbb{R}^{+}$ó $\mathbb{R}$ se dice que es estacionario en sentido estricto si las dos distribuciones finito dimensionales del proceso son invariantes bajo traslaciones de tiempo, es decir,

$$
P_{X_{t_{1}}, \ldots, X_{t_{n}}}(C)=P_{X_{t_{1}+h}, \ldots, X_{t_{n}+h}}(C)
$$

Para toda $t_{1}, \ldots, t_{n}, h \in T$ y para cualquier conjunto medible $C \subset S^{n}$.

\subsection{Procesos Estocásticos de Segundo Orden}

Definición 13. Un proceso estocástico complejo ó real valuado se dice que es estacionario en sentido amplio ó de segundo orden si las variables aleatorias $\left\{X_{t}\right\}_{t \in T}$ tienen segundos momentos los cuales son invariantes bajo traslaciones del tiempo con $X_{t}: \Omega \longrightarrow \mathbb{C}$ ó $\mathbb{R} ;$ para ser más precisos

i) $E\left(X_{t+h}\right)=E\left(X_{t}\right)$ para toda $t, h \in T$. 
ii) $E\left(X_{t+h} \bar{X}_{s+h}\right)=E\left(X_{t} \bar{X}_{s}\right)$ para toda $t, s, h \in T$.

Si un proceso estocástico estacionario en sentido estricto tiene segundos momentos entonces es estacionario en sentido amplio pero no a la inversa, ver Teorema 6.

Consideremos procesos con segundo momento finito. Las variables del proceso $\left\{X_{t}\right\}_{t \in T}$ las podemos pensar como elementos en el espacio de Hilbert $H=L_{2}(\Omega, \mathfrak{F}, P)$. Definimos

$$
\|X\|=\sqrt{E\left(|X|^{2}\right)} \quad \text { y } \quad\langle X, Y\rangle=E(X \bar{Y})
$$

y la Función Covarianza del proceso se define como

$$
K(s, t)=E\left(X_{s} \bar{X}_{t}\right)=\left\langle X_{s}, X_{t}\right\rangle
$$

Definición 14. Una matriz $K$ se dice que es no negativa definida ó que es semidefinida positiva si es una matriz hermitiana cuadrada $n \times n$ donde para todo $x \in \mathbb{C}^{n}$ tenemos que $\bar{x} K x \geq 0$.

Teorema 5. Una función $K: T \times T \longrightarrow \mathbb{C}$ es la covarianza de algún proceso $\left\{X_{t}\right\}_{t \in T}$ si y sólo si $K$ es no negativa definida.

Demostración. Para la necesidad, observemos que si $K$ es función de covarianza de $\left\{X_{t}\right\}_{t \in T}$, entoces

$$
K=\left(\begin{array}{cccc}
E\left(X_{t_{1}}^{2}\right) & E\left(X_{t_{1}} X_{t_{2}}\right) & \ldots & E\left(X_{t_{1}} X_{t_{n}}\right) \\
\vdots & \vdots & \ddots & \vdots \\
E\left(X_{t_{n}} X_{t_{1}}\right) & E\left(X_{t_{n}} X_{t_{2}}\right) & \ldots & E\left(X_{t_{n}}^{2}\right)
\end{array}\right)
$$

con $\alpha_{1}, \ldots, \alpha_{n} \in \mathbb{C}$ se tiene que

$$
\left(\alpha_{1} \ldots \alpha_{n}\right)\left(\begin{array}{cccc}
E\left(X_{t_{1}}^{2}\right) & E\left(X_{t_{1}} X_{t_{2}}\right) & \ldots & E\left(X_{t_{1}} X_{t_{n}}\right) \\
\vdots & \vdots & \ddots & \vdots \\
E\left(X_{t_{n}} X_{t_{1}}\right) & E\left(X_{t_{n}} X_{t_{2}}\right) & \ldots & E\left(X_{t_{n}}^{2}\right)
\end{array}\right)\left(\begin{array}{c}
\alpha_{1} \\
\vdots \\
\alpha_{n}
\end{array}\right)=E\left|\sum_{i=1}^{d} \alpha_{i} X_{i}\right|^{2} \geq 0 .
$$

Por tanto $K$ es no negativa definida. Para la suficiencia se debe construir un proceso $\left\{X_{t}\right\}_{t \in T}$ con función de covarianza K. Ver[3] pp. 26-28.

Ejemplo 3. Consideremos una función aleatoria $X:[0, \infty) \times \Omega \longrightarrow \mathbb{C} X_{t}=f(t) A(w)$ donde $f(t)$ no es aleatoria y $A(w)$ no depende de $t$. Si $0<E\left(|A|^{2}\right)<\infty$, entonces $\left\{X_{t}\right\}_{t \in T}$ es estacionario en sentido amplio si y sólo si $f(t+s) \overline{f(t)}$ depende solamente de $s \forall t, s \in T$. 
Demostración. Mostraremos primero la necesidad. Supongamos que $\left\{X_{t}\right\}_{t \in T}$ es estacionario en sentido amplio, por tanto

$$
E\left(X_{t+s} \bar{X}_{t}\right)=K(s)
$$

Pero $X_{t+s}=f(t+s) A(w)$ y $\bar{X}_{t}=\overline{f(t) A(w)}$, asi;

$$
\begin{aligned}
E\left(X_{t+s} \bar{X}_{t}\right) & =E(f(t+s) \overline{f(t)} A \bar{A}) \\
& =f(t+s) \overline{f(t)} E(A \bar{A}) \\
& =f(t+s) \overline{f(t)} E\left(|A|^{2}\right)=K(s)
\end{aligned}
$$

por lo tanto $f(t+s) \overline{f(t)}$ sólo depende de $s$ para todo $t, s \in T$.

Para la suficiencia, si $f(t+s) \overline{f(t)}$ sólo depende de $s$, entonces

$$
E\left(X_{t+s} \bar{X}_{t}\right)=f(t+s) \overline{f(t)} E\left(|A|^{2}\right)=K(s)
$$

Por tanto $\left\{X_{t}\right\}_{t \in T}$ es estacionario en sentido amplio.

Ahora probemos que las únicas funciones $f(t)$ que son como en el ejemplo anterior son las funciones $f(t)=B e^{i L t}$ con $B \in \mathbb{C}, B \neq 0$ y $L$ constante real.

Demostración. Sea $f(t)=B e^{i t L}$, entonces de la relación ya demostrada

$$
\frac{f(t+s)}{B}=\frac{f(t)}{B} \frac{f(s)}{B}
$$

se obtiene que

$$
\frac{f(t+s) \overline{f(t)}}{B}=\frac{|f(t)|^{2}}{B} \frac{f(s)}{B} .
$$

Pero $|f(t)|^{2}=B^{2}$ entonces queda

$$
\frac{f(t+s) \overline{f(t)}}{B}=f(s)
$$

lo cual demuestra que la función satisface la condición deseada. Reciprocamente, si $f(t+s) \overline{f(t)}$ depende sólo de s para todo $s, t$, entonces como se demostró en el ejemplo anterior, se tiene que

$$
f(t+s) \overline{f(t)}=C K(s)
$$

donde $C=E\left(|A|^{2}\right)$ es una constante.

Ahora bien, con $s=0$ en la expresión anterior se tiene $|f(t)|^{2}=C K(0)$ para toda $t$, entonces $f(t)=B e^{i t L}$ con $B=C K(0)$ y alguna constante real $L$. 
Ejemplo 4. Si $A_{1} \ldots, A_{n}$ son ortogonales con $X_{t}=\sum_{j=1}^{n} A_{j} e^{i \lambda_{j} t}$ con $\lambda_{j}$ real, $\left\{X_{t}\right\}_{t \in T}$ es un proceso estacionario en sentido amplio.

Demostración. Si $A_{1}, \ldots, A_{n}$ son ortogonales, entonces $E\left(A_{i} A_{j}\right)=0 \forall i \neq j$. Por tanto

$$
\begin{aligned}
E\left(X_{t+h} \bar{X}_{s+h}\right) & =E\left(\sum_{j=1}^{n} A_{j} e^{i \lambda_{j}(t+h)} \sum_{k=1}^{n} \bar{A}_{k} e^{i \lambda_{k}(s+h)}\right) \\
& =E\left(\sum_{j=1}^{n} \sum_{k=1}^{n} \bar{A}_{k} A_{j} e^{i\left(\lambda_{j}(t+h)-\lambda_{k}(s+h)\right)}\right) \\
& =\sum_{j=1}^{n} E\left(\left|A_{j}\right|^{2} e^{i \lambda_{j}(t-s)}\right) \\
& =\sum_{j=1}^{n} \sum_{k=1}^{n} E\left(A_{j} \bar{A}_{k} e^{i \lambda_{j} t} e^{i \lambda_{j} s}\right) \\
& =E\left(X_{t} \bar{X} s\right)
\end{aligned}
$$

Por tanto $\left\{X_{t}\right\}_{t \in T}$ es estacionario en sentido amplio.

Ejemplo 5. Sea $\left\{\xi_{n}\right\}_{n \in \mathbb{Z}}$ sucesión ortonormal, los "promedios móviles" se definen como:

$$
X_{n}=\sum_{k=-\infty}^{\infty} C_{k} \xi_{n-k}
$$

con $\sum_{k=0}^{\infty}\left|C_{k}\right|^{2}<\infty$, para que la serie converja. Entonces $\left\{X_{n}\right\}_{n \in \mathbb{Z}}$ es estacionario en sentido amplio.

Demostración. Tenemos que

$$
\begin{aligned}
E\left(X_{t+h} \bar{X}_{s+h}\right) & =E\left(\sum_{k=-\infty}^{\infty} C_{k} \xi_{t+h-k} \sum_{j=-\infty}^{\infty} \bar{C}_{j} \bar{\xi}_{s+h-j}\right) \\
& =E\left(\sum_{k=-\infty}^{\infty} \sum_{j=-\infty}^{\infty} C_{k} \bar{C}_{j} \xi_{t+h-k} \bar{\xi}_{s+h-j}\right) \\
& =\sum_{k=-\infty}^{\infty} \sum_{j=-\infty}^{\infty} E\left(C_{k} \bar{C}_{j} \xi_{t+h-k} \bar{\xi}_{s+h-j}\right) \\
& =\sum_{k=-\infty}^{\infty} \sum_{j=-\infty}^{\infty} E\left(C_{k} \bar{C}_{j} \xi_{t-k} \bar{\xi}_{s-j}\right) \\
& =E\left(X_{t} \bar{X}_{s}\right)
\end{aligned}
$$

Por lo tanto $\left\{X_{n}\right\}_{n \in \mathbb{Z}}$ es estacionario en sentido amplio. 
Teorema 6. Si un proceso $X$ es estacionario en sentido estricto y tiene segundo momento entonces es estacionario en sentido amplio.

Demostración. Tenemos que $X$ es estacionario en sentido estricto y tiene segundo momento finito, por tanto

$$
\begin{aligned}
E\left(X_{t+h}\right) & =\int_{\mathbb{C}} x d P_{X_{t+h}} \\
& =\int_{\mathbb{C}} x d P_{X_{t}} \\
& =E\left(X_{t}\right)
\end{aligned}
$$

Para toda $t, s, h \in T, y$

$$
\begin{aligned}
E\left(X_{t+h} \bar{X}_{s+h}\right) & =\int_{\mathbb{C}^{2}} x \bar{y} d P_{X_{t+h}, \bar{X}_{s+h}} \\
& =\int_{\mathbb{C}^{2}} x \bar{y} d P_{X_{t} \bar{X}_{s}} \\
& =E\left(X_{t} \bar{X}_{s}\right)
\end{aligned}
$$

Finalmente

$$
E\left(X_{t+s} \bar{X}_{t}\right)=\int_{\mathbb{C}^{2}} x \bar{y} d P_{X_{t+s} \bar{X}_{t}}=\int_{\mathbb{C}^{2}} x \bar{y} d P_{X_{s}, X_{0}}=K(s)
$$

\subsubsection{Construcción de un proceso estacionario en sentido amplio}

Sea $H=L_{2}(\Omega, \mathfrak{F}, P)$ y $U: H \longrightarrow H$ operador unitario, es decir, un operador lineal cuyo rango es un conjunto denso y para todo vector y y $x$ de $H$ se tiene que $\langle U x, U y\rangle=\langle x, y\rangle$ sobre un subespacio cerrado $M \subset H$, es decir, $U$ está definido sólo en $M$. Tomando cualquier vector $x \in M$, la sucesión $\left\{X_{n}\right\}_{n \in \mathbb{Z}}$, donde $X_{n}=U^{n} x$ para todo $n \in \mathbb{Z}$, es un proceso estacionario en sentido amplio pues:

$$
E\left(X_{n+m} \bar{X}_{n}\right)=\left\langle U^{n+m} x, U^{n} x\right\rangle=\left\langle U^{m} x, x\right\rangle=K(m) .
$$

Recíprocamente ¿Cómo construir un operador unitario cuando sólo tenemos el proceso estacionario?, para ésto tenemos dos casos, el caso discreto y el caso continuo. 
1. Sea $T=\mathbb{Z}$. Dado $\left\{X_{n}\right\}_{n \in T}$ proceso estacionario en sentido amplio, sea $M$ el subespacio cerrado más pequeño de $H$ el cual contiene a todos los vectores $\left\{X_{n}\right\}$. Sea $M^{1} \subset M$ el conjunto de todas las combinaciones lineales finitas de $\left\{X_{n}\right\}$, entonces $M=\overline{\operatorname{span}}\left\{X_{n}\right\}_{n \in T}$, es decir, la cerradura de $M^{1}$.

Como $\left\{X_{n}\right\}_{n \in Z} \subset L_{2}(\Omega, \mathfrak{F}, P)$ es estacionario en sentido amplio, y

$$
E\left(X_{n+m} \bar{X}_{n}\right)=K(m)=\left\langle X_{n+m}, X_{n}\right\rangle
$$

Sea $U X_{n}=X_{n+1}$ para toda $n \in \mathbb{Z}$ entonces

$$
\left\langle U X_{n+m}, U X_{n}\right\rangle=\left\langle X_{n+m+1}, X_{n+1}\right\rangle=K(m)=\left\langle X_{n+m}, X_{n}\right\rangle
$$

$U$ es una transformación entre las variables del proceso que respeta el producto interno y si suponemos que $M$ es denso en $\mathrm{H}$, entonces se puede extender linealmente a un operador lineal sobre $M$ que es unitario.

2. Ahora, si $T=\mathbb{R}$, sea $\left\{X_{t}\right\}_{t \in T}$ proceso estacionario en sentido amplio, $M=\overline{\operatorname{span}}\left\{X_{t}\right\}_{t \in T} \subset$ $L_{2}(\Omega, \mathfrak{F}, P)$ con $M$ denso en $H$. Para $t \in T$ fijo si $U_{t}$ se define en $M$ como

$$
U_{t} X_{s}=X_{t+s} \quad \forall t, s \in T
$$

entonces se tiene que

$$
\left\langle U_{t} X_{s+h}, U_{t} X_{s}\right\rangle=K(h)=\left\langle X_{s+h}, X_{s}\right\rangle
$$

entonces $U_{t}$ se extiende a un operador lineal unitario sobre $M$. La familia $\left\{U_{t}\right\}_{t \in T}$ es un grupo de operadores unitarios pues $X_{0} \in M$ y

$$
U_{t} U_{h} X_{s}=U_{t} X_{h+s}=X_{t+h+s}=U_{t+h} X_{s}
$$

por tanto

$$
U_{t} U_{h}=U_{t+h} \quad \forall t, h \in T .
$$

Para procesos estacionarios de segundo orden se tienen las siguientes propiedades importantes.

Proposición 2. Sea $\left\{X_{n}\right\}_{n \in \mathbb{Z}}$ estacionario es sentido amplio. Entonces existe $Y \in L_{2}$ tal que

$$
\lim _{n-m \rightarrow \infty} \frac{1}{n-m} \sum_{j=m}^{n-1} X_{j}=Y
$$

en la norma $L_{2}$.

Demostración. Ver[3] pg. 40. 
Corolario 2. Tenemos que $Y=0$ si y sólo si

$$
\frac{1}{N} \sum_{j=0}^{N-1} K(j) \longrightarrow 0
$$

donde $K$ es la función de Covarianza de $\left\{X_{n}\right\}_{n \in \mathbb{Z}}$.

Demostración. Utilizando la proposición anterior, para cualquier $m \in \mathbb{Z}$

$$
\begin{aligned}
\left\langle Y, X_{m}\right\rangle & =\left\langle\lim _{n \rightarrow \infty} \frac{1}{n-m} \sum_{j=m}^{n-1} X_{j}, X_{m}\right\rangle \\
& =E\left(\lim _{n \rightarrow \infty} \frac{1}{n-m} \sum_{j=m}^{n-1} X_{j} X_{m}\right) \\
& =\lim _{n \rightarrow \infty} \frac{1}{n-m} \sum_{j=m}^{n-1} E\left(X_{j} X_{m}\right) \quad \text { si } N=n-m \\
& =\lim _{N \rightarrow \infty} \frac{1}{N} \sum_{j=0}^{N-1} E\left(X_{j+m} X_{m}\right) \\
& =\lim _{N \rightarrow \infty} \frac{1}{N} \sum_{j=0}^{N-1} K(j)
\end{aligned}
$$

Puesto que el limite existe. Si $Y=0$ entonces $\lim _{N \rightarrow \infty} \frac{1}{N} \sum_{j=0}^{N-1} K(j)=0$. Inversamente si $\lim _{N \rightarrow \infty} \frac{1}{N} \sum_{j=0}^{N-1} K(j)=0$, entonces $\left\langle Y, X_{m}\right\rangle=0$ para cada $m \in \mathbb{Z}$, pero $Y$ pertenece al subespacio generado por $\left\{X_{m}\right\}_{m \in \mathbb{Z}}$, por tanto $Y=0$.

\subsection{Procesos Estacionarios en Sentido Estricto}

Consideremos los siguientes ejemplos de procesos estacionarios en sentido estricto.

Ejemplo 6. Sea $A$ una variable aleatoria para toda $t \in T$, observemos que $X_{t}=A(\omega)$ es estacionaria, pero $X_{t}=e^{i \lambda t} A$ no siempre es estacionaria. Sin embargo si

$$
X_{t}=A e^{i(\lambda t+\phi)}
$$


donde $A$ es real y $\phi$ es variable aleatoria con distribución uniforme sobre $[-\pi, \pi]$ e independiente de $A$, entonces $\left\{X_{t}\right\}$ es estacionario.

Demostración. Observemos que a las variables $X_{t}$ las podemos ver como

$$
\gamma_{(\omega)}(t)=(A(\omega) \cos (\lambda t+\phi), A(\omega) \sin (\lambda t+\phi))
$$

con $t, h \in \mathbb{R}$, así

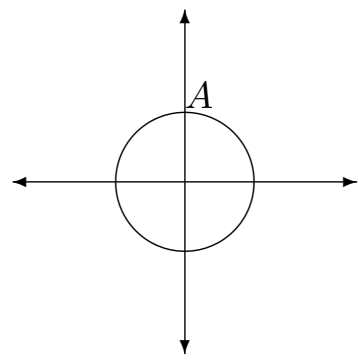

Imagen $\gamma(I)$

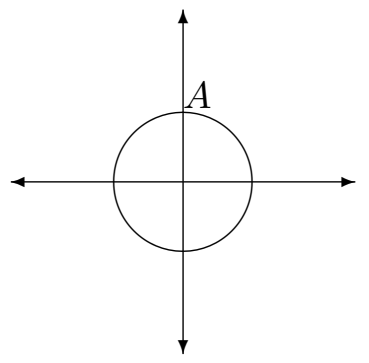

Imagen $\gamma(I+h)$

Consideremos coordenadas polares, por lo tanto sean $B_{i}=\left[R_{1}^{i}, R_{2}^{i}\right] \times\left[\theta_{1}^{i}, \theta_{2}^{i}\right]$. Si $i=1$ se tiene que

$$
\begin{aligned}
P\left(X_{t} \in B_{1}\right)=P\left(\gamma_{\omega}(t) \in B_{1}\right) & =P\left(A \in\left[R_{1}^{1}, R_{2}^{1}\right],(\lambda t+\phi) \in\left[\theta_{1}^{1}, \theta_{2}^{1}\right]\right) \\
& =P\left(A \in\left[R_{1}^{1}, R_{2}^{1}\right], \phi \in\left[\theta_{1}^{1}-\lambda t, \theta_{2}^{1}-\lambda t\right]\right) \\
& =P\left(A \in\left[R_{1}^{1}, R_{2}^{1}\right]\right) P\left(\phi \in\left[\theta_{1}^{1}-\lambda t, \theta_{2}^{1}-\lambda t\right]\right) \\
& =P\left(A \in\left[R_{1}^{1}, R_{2}^{1}\right]\right)\left(\theta_{1}^{1}-\theta_{2}^{1}\right) \\
& =P\left(X_{t+h} \in B_{1}\right)
\end{aligned}
$$

Si $i=2$ se tiene que

$$
\begin{aligned}
P\left(\left(X_{t_{1}}, X_{t_{2}}\right) \in B_{1} \times B_{2}\right)= & P\left(A \in\left[R_{1}^{1}, R_{2}^{1}\right], A \in\left[R_{1}^{2}, R_{2}^{2}\right],\left(\lambda t_{1}+\phi\right) \in\left[\theta_{1}^{1}, \theta_{2}^{1}\right],\left(\lambda t_{2}+\phi\right) \in\left[\theta_{1}^{2}, \theta_{2}^{2}\right]\right) \\
= & P\left(A \in \cap_{i=1}^{2}\left[R_{1}^{i}, R_{2}^{i}\right], \phi \in\left[\theta_{1}^{1}-\lambda t_{1}, \theta_{2}^{1}-\lambda t_{1}\right] \cap\left[\theta_{1}^{2}-\lambda t_{2}, \theta_{2}^{2}-\lambda t_{2}\right]\right) \\
= & P\left(A \in \cap_{i=1}^{2}\left[R_{1}^{i}, R_{2}^{i}\right]\right) P\left(\phi \in\left[\theta_{1}^{1}-\lambda t_{1}, \theta_{2}^{1}-\lambda t_{1}\right] \cap\left[\theta_{1}^{2}-\lambda t_{2}, \theta_{2}^{2}-\lambda t_{2}\right]\right) \\
= & P\left(A \in \cap_{i=1}^{2}\left[R_{1}^{i}, R_{2}^{i}\right]\right) P\left(\phi \in [ \theta _ { 1 } ^ { 1 } - \lambda ( t _ { 1 } + h ) , \theta _ { 2 } ^ { 1 } - \lambda ( t _ { 1 } + h ) ] \cap \left[\theta_{1}^{2}-\right.\right. \\
& \left.\left.\lambda\left(t_{2}+h\right), \theta_{2}^{2}-\lambda\left(t_{2}+h\right)\right]\right) \\
= & P\left(\left(X_{t_{1}+h}, X_{t_{2}+h}\right) \in B_{1}, B_{2}\right)
\end{aligned}
$$

Así se sigue con las distribuciones finito dimensionales (cuando $i=n$ ), para finalmente concluir que $\left\{X_{t}\right\}_{t \in T}$ es un proceso estacionario en sentido estricto.

Ejemplo 7. Sea $T=\mathbb{Z}$. Una sucesión independiente $\left\{\xi_{n}\right\}_{n \in T}$ es un proceso estacionario, si y sólo si las variables aleatorias son distribuidas identicamente. 
Demostración. Para la necesidad, si $\left\{\xi_{n}\right\}$ es estacionario, entonces $P\left(\left(\xi_{1}, \ldots, \xi_{n}\right) \in C\right)=$ $P\left(\left(\xi_{1+h}, \ldots, \xi_{n+h}\right) \in C\right)$. Si $n=1, P\left(\xi_{1} \in C\right)=P\left(\xi_{1+h} \in C\right)$ para toda $h \in \mathbb{Z}$, es decir, $\left\{\xi_{n}\right\}$ son distribuidas identicamente.

Para la suficiencia, si $\left\{\xi_{n}\right\}$ son distribuidas identicamente, entoces

$$
\begin{aligned}
P\left(\left(\xi_{1}, \ldots, \xi_{n}\right) \in C\right) & =P\left(\xi_{1} \in A_{1}\right), \ldots, P\left(\xi_{n} \in A_{n}\right) \\
& =P\left(\xi_{1+h} \in A_{1}\right), \ldots, P\left(\xi_{n+h} \in A_{n}\right) \\
& =P\left(\left(\xi_{i+h}, \ldots, \xi_{n+h}\right) \in C\right)
\end{aligned}
$$

Así, $\left\{\xi_{n}\right\}$ es estacionario.

Ejemplo 8. Si $X_{0}, X_{1}, \ldots$ es una sucesión estacionaria y $g: \mathbb{R}^{\mathbb{N}} \longrightarrow \mathbb{R}$ es medible, entonces $Y_{k}=g\left(X_{k}, X_{k+1}, \ldots\right)$ es estacionaria.

Demostración. Si $x \in \mathbb{R}^{\mathbb{N}}$, entonces $g_{k}(x)=g\left(x_{k}, x_{k+1}, \ldots\right)$ y si $B \in \mathfrak{B R}^{\mathbb{N}}$, sea $A=\{x$ : $\left.\left(g_{0}(x), g_{1}(x), \ldots\right) \in B\right\}$. Asi

$$
\begin{aligned}
P\left(\left\{\omega:\left(Y_{0}, Y_{1}, \ldots\right) \in B\right\}\right) & =P\left(\left\{\omega:\left(X_{0}, X_{1}, \ldots\right) \in B\right\}\right) \\
& =P\left(\left\{\omega:\left(X_{k}, X_{k+1}, \ldots\right) \in B\right\}\right) \\
& =P\left(\left\{\omega:\left(Y_{k}, Y_{k+1}, \ldots\right) \in B\right\}\right)
\end{aligned}
$$

\subsection{Teorema Ergódico}

Definición 15. Sea $(\Omega, \mathfrak{F}, P)$ un espacio de probabilidad y supongamos que $\Psi: \Omega \longrightarrow \Omega$ es una transformación medible. Decimos que $\Psi$ preserva la medida siempre que

$$
P\left(\Psi^{-1} A\right)=P(A) \quad \forall A \in \mathfrak{F}
$$

Si $\Psi$ tiene inversa, la inversa automaticamente preserva la medida y $\Psi$ es llamada invertible.

Teorema 7. (Teorema Ergódico de Birkhoff). Sea $\Psi$ una transformación que preserva la medida sobre un espacio de probabilidad $(\Omega, \mathfrak{F}, P)$, y sea $f \in L_{1}$. Entonces

$$
\lim _{n \rightarrow \infty} \frac{1}{n} \sum_{k=0}^{n-1} f\left(\Psi^{k} \omega\right)=\tilde{f}(\omega)
$$

existe casi dondequiera en $L_{1}$. Más aún, $\tilde{f} \in L_{1}$ y $\int_{A} \tilde{f}=\int_{A} f$ para toda $A \in \mathfrak{F}_{I}=\{C \in \mathfrak{F}$ : $\left.\Psi^{-1} C=C\right\}$, donde $\mathfrak{F}_{I}$ es llamada la sigma-álgebra de los conjuntos invariantes. 
Demostración. Ver [3].

Si consideramos un proceso estacionario en sentido estricto para el teorema Ergódico obtenemos el siguiente teorema.

Teorema 8. (Ley de los Grandes Números para Procesos Estacionarios). Sea $T=\mathbb{Z}$ $y\left\{X_{n}\right\}_{n \in T}$ un proceso estacionario en sentido estricto con $E\left(\left|X_{n}\right|\right)<\infty$. Entonces

$$
\lim _{n \rightarrow \infty} \frac{1}{n} \sum_{k=0}^{n-1} X_{k}=\tilde{X}
$$

existe casi seguramente y en $L_{1}$. El límite $\tilde{X}$ es integrable y $E(\tilde{X})=E\left(X_{0}\right)$.

Observemos que si $E\left(X_{n}^{2}\right)<\infty$, entonces $\left\{X_{n}\right\}$ es estacionario en sentido amplio y por tanto según la Proposición 2

$$
\lim _{N \rightarrow \infty} \frac{1}{N} \sum_{j=0}^{N-1} X_{j}=Y, \quad \text { así } \tilde{X}=Y .
$$

Para procesos estacionarios en tiempo continuo tenemos el siguiente teorema:

Teorema 9. Sea $\left\{X_{t}\right\}_{t \in T}, T=\mathbb{R}$ un proceso estacionario medible y supongamos que $E\left(\left|X_{t}\right|\right)<$ $\infty$. Entonces

$$
\lim _{N \rightarrow \infty} \frac{1}{N} \int_{0}^{N} X_{t} d t=\tilde{X}
$$

existe casi seguramente. Además $\tilde{X}$ es integrable y $E(\tilde{X})=E\left(X_{0}\right)$

El Teorema Ergódico implica el Teorema 8 y la prueba es la siguiente:

Demostración. Sea $(\Omega, \mathfrak{F}, P)$ espacio de probabilidad con $\Omega=\mathbb{R}^{\mathbb{N}}$ y $\mathfrak{F}=\mathcal{C}$ la $\sigma$-álgebra cilíndrica como en el Teorema de Consistencia de Kolmogorov. Sea $\Psi\left(a_{0} a_{1} \ldots\right)=\left(a_{1} a_{2} \ldots\right)$. Observemos que $\Psi^{k}\left(a_{0} a_{1} \ldots\right)=\left(a_{k} a_{k+1} \ldots\right)$ y además $X_{k}\left(a_{0} a_{1} \ldots\right)=a_{k}$ donde $X_{j} \in L_{1}$ para toda $j$. Por tanto

$$
\begin{aligned}
X_{0}\left(\Psi^{k}(\omega)\right) & =X_{0}\left(\Psi^{k}\left(a_{0} a_{1} \ldots\right)\right) \\
& =X_{0}\left(a_{k} a_{k+1} \ldots\right) \\
& =a_{k} \\
& =X_{k}\left(a_{0} a_{1} \ldots\right) \\
& =X_{k}(\omega)
\end{aligned}
$$

Entonces probemos que $\Psi$ es una transformación que preserva la medida. Tenemos pues que $\Psi: \Omega \longrightarrow \Omega$, es decir, $\Psi: \mathbb{R}^{\mathbb{N}} \longrightarrow \mathbb{R}^{\mathbb{N}}$. 
1. $\Psi$ es medible.

Sea $D \in \mathcal{C}$. Supongamos que $D$ tiene la siguiente forma $D=\left\{\left(a_{0}, a_{1}, \ldots\right): a_{i_{0}} \in B\right\}=$ $\left\{X_{i_{1}} \in B\right\}$ donde $i_{0} \in\{0,1,2, \ldots\}$ fijo, así

$$
\Psi^{-1}(D)=\left\{\left(a_{0} a_{1} \ldots\right): a_{i_{0}+1} \in B\right\}=\left\{X_{i_{0}+1} \in B\right\}
$$

Donde éstos $D$ generan a $\mathcal{C}$.

2. $P\left(\Psi^{-1}(D)\right)=P(D)$

$$
\begin{aligned}
P\left(\Psi^{-1}(D)\right) & =P\left(X_{i_{0}+1} \in D\right) \\
& =P\left(X_{i_{0}} \in D\right) \\
& =P(D)
\end{aligned}
$$

Por tanto

$$
\lim _{n \rightarrow \infty} \frac{1}{n} \sum_{k=0}^{n-1} X_{k}=\lim _{n \rightarrow \infty} \frac{1}{n} \sum_{k=0}^{n-1} X_{0}\left(\Psi^{k}(\omega)\right)=E\left(X_{k} \mid \mathfrak{F}_{I}\right)
$$

Y el Teorema 8 implica la Ley de los Grandes Números.

Teorema 10. (Ley de los Grandes Números). Sean $X_{1}, X_{2}, \ldots$ variables aleatorias independientes e identicamente distribuidas, con $E\left(\left|X_{i}\right|\right)<\infty$ y $E\left(X_{0}\right)=\mu$, entonces

$$
\lim _{n \rightarrow \infty} \frac{1}{n} \sum_{k=0}^{n-1} X_{k}=\mu
$$

casi dondequiera y en $L_{1}$.

Demostración. Por lo anterior sólo basta mostrar que $E\left(X_{0} \mid \mathfrak{F}_{I}\right)=E\left(X_{0}\right)=$ constante, es decir, $\mathfrak{F}_{I} \subset \tau$, donde $\tau:=\bigcap_{k=0}^{\infty} \tau_{k}$ con $\tau_{k}=\sigma\left(X_{k+1}, X_{k+2}, \ldots\right)$. Observemos que $\tau_{0}=$ $\sigma\left(X_{1}, X_{2}, \ldots\right)=\mathcal{C}$.

Sea $A \in \mathfrak{F}_{I}$, entonces queremos que $A \in \tau=\bigcup_{k=0}^{\infty} \tau_{k}$, es decir, $A \in \tau_{k} \forall k \in\{0,1,2, \ldots\}$. $Y$ tenemos que $\mathfrak{F}_{I}=\left\{C \in \mathcal{C}: \Psi^{-1} C=C\right\}$. Si $\tau_{0}=\mathcal{C}$ entonces $A \in \tau_{0}$. Sea $A=\left(X_{0}(\omega) \in\right.$ $\left.A_{0}, X_{1}(\omega) \in A_{1}, \ldots\right)$ :

$$
\begin{aligned}
\Psi^{-1} A & =\Psi^{-1}\left(X_{0}(\omega) \in A_{0}, X_{1}(\omega) \in A_{1}, \ldots, X_{n}(\omega) \in A_{n}\right) \\
& =\left\{\omega \in \Omega:\left(\omega_{1}, \omega_{2}, \ldots\right) \in A\right\} \\
& =\left\{\omega \in \Omega:\left(\omega_{1} \in A_{0}, \omega_{2} \in A_{1} \ldots\right)\right\} \\
& =\left(X_{1}(\omega) \in A_{0}, X_{2}(\omega) \in A_{2}, \ldots\right) \in \tau_{1}
\end{aligned}
$$


Por tanto, si $A \in \tau_{l}$ entonces

$$
\begin{aligned}
\Psi^{-k} A & =\Psi^{-1}\left(X_{l} \in A_{0}, X_{l+1} \in A_{1}, \ldots, X_{l+n} \in A_{n}\right) \\
& =\left\{\omega \in \Omega:\left(\omega_{l+k} \in A_{0}, \omega_{l+k+1} \in A_{1}, \ldots\right)\right\} \\
& =\left(X_{l+k}(\omega) \in A_{0}, X_{l+k+1}(\omega) \in A_{1}, \ldots\right) \in \tau_{l+k}
\end{aligned}
$$

Si $A \in \mathfrak{F}_{I}$ entonces $A \in \tau_{l}$, por lo tanto $A \in \tau_{k} \forall k$ que finalmente concluye que $A \in \tau$. Por lo tanto $\mathfrak{F}_{I} \subset \tau$. Por la Ley $0-1$ de Kolmogorov si $A \in \mathfrak{F}_{I}$ entonces $P(A)=0$ ó $P(A)=1$ entonces $E\left(X_{0} \mid \mathfrak{F}_{I}\right)$ es constante. 


\section{Capítulo 3}

\section{Balance Detallado}

En este Capítulo estudiaremos lo que es la noción de Balance Detallado para procesos estocásticos con la propiedad de Markov tanto en tiempo discreto como en tiempo continuo donde el espacio de estados $S$ es un espacio métrico compacto y $\mu$ una medida invariante con $p$ núcleo estocástico.

\subsection{Cadenas de Markov en tiempo discreto.}

Consideraremos procesos estocásticos a tiempo discreto, tales que tienen la propiedad de Markov. El conjunto $T$ será igual a $\mathbb{N}$ y el espacio de estados $S$ un espacio métrico compacto. El espacio de probabilidad será $(\Omega, \mathfrak{F}, P)$.

Definición 16. Se dice que $\left\{X_{n}\right\}_{n \in T}$ es una cadena de Markov con respecto a la filtración $\left\{\mathfrak{F}_{n}\right\}_{n \in T}$ si:

1. $\left\{X_{n}\right\}_{n \in T}$ es adaptado a $\left\{\mathfrak{F}_{n}\right\}_{n \in T}$.

2. Para cada $n<t, A \in \mathfrak{S}$.

$$
P\left(X_{t} \in A \mid \mathfrak{F}_{n}\right)=P\left(X_{t} \in A \mid X_{n}\right)
$$

La propiedad 2 se llama propiedad de Markov. 
Definición 17. Sea $\left\{p_{n}\right\}_{n \in T}$ una sucesión de núcleos estocásticos. Supóngase que $\left\{X_{n}\right\}_{n \in T}$ es una cadena de Markov respecto a $\left\{\mathfrak{F}_{n}\right\}_{n \in T}$. Decimos que $\left\{p_{n}\right\}_{n \in T}$ es la función de transición de $\left\{X_{n}\right\}_{n \in T}$ si $m<n$,

$$
P\left[X_{n} \in B \mid \mathfrak{F}_{m}\right]=p_{n m}\left(X_{m}, B\right) .
$$

Si $S$ es a lo más numerable, entonces la función de transición se puede ver de forma matricial:

$$
P=\left(\begin{array}{ccccc}
p(0,0) & p(0,1) & \ldots & p(0, k) & \ldots \\
p(1,0) & p(1,1) & \ldots & p(1, k) & \ldots \\
\vdots & \vdots & \ddots & \vdots & \vdots \\
p(k, 0) & p(k, 1) & \ldots & p(k, k) & \ldots
\end{array}\right)
$$

Además si $p_{n}\left(X_{n}, B\right)$ no depende de $n$, entonces se dice que la función de transición de la cadena de Markov es estacionaria. La integral que define a una medida invariante $\mu$ en tiempo discreto se reduce a calcular una suma, por tanto $\mu=(\mu(0), \mu(1), \ldots)$ es medida invariante de una cadena de Markov con probabilidad de transición $p(x, y)$ si $\mu(y)=\sum_{x} \mu(x) p(x, y)$.

Definición 18. Si $S$ es a lo más numerable, diremos que $\mu$ está en balance detallado respecto al núcleo estocástico $p$ si

$$
\mu(x) p(x, y)=\mu(y) p(y, x)
$$

Para todo $x, y \in S$.

Si $\mu$ cumple la condición de balance detallado, también se le llama medida reversible. Observemos que si $\mu$ está en balance detallado respecto a $p$, entonces $\mu$ es invariante. La prueba es sencilla:

$$
\begin{aligned}
\sum_{x} \mu(x) p(x, y) & =\sum_{x} \mu(y) p(y, x) \quad \text { por estar en balance detallado } \\
& =\mu(y) \sum_{x} p(y, x) \\
& =\mu(y)
\end{aligned}
$$

Sin embargo, si $\mu$ es medida invariante no implica que $\mu$ esté en balance detallado lo cual se muestra con los ejemplos.

Definición 19. Sea $t \in T$. Se dice que un estado $x \in S$ es recurrente si

$$
P\left(\bigcup_{n=1}^{\infty}\left(X_{n}=x\right) \mid X_{0}=x\right)=1, \quad \text { para alguna } n \geq 1 .
$$

Un estado $x \in S$ es transitorio si y sólo si no es recurrente. 


\section{Definición 20.}

1. Un estado $x \in S$ es llamado absorbente si $P\left(X_{n+1}=x \mid X_{n}=x\right)=1$.

2. Dos estados $x, y \in S$ se comunican si para algún $n, P\left(X_{n}=y \mid X_{0}=x\right)>0$ y para algún $m, P\left(X_{m}=x \mid X_{0}=y\right)>0$.

3. Si $\boldsymbol{T}_{x}=\operatorname{mín}\left\{n \leq 1: X_{n}=x\right\}$ con $x \in S$, decimos que $x$ es recurrente positivo si $E\left[\boldsymbol{T}_{x} \mid X_{0}=x\right]<\infty$.

Si todos los estados de una cadena de Markov se comunican, entonces decimos que la cadena es irreducible.

Un ejemplo de la clasificación de estados es el siguiente diagrama.

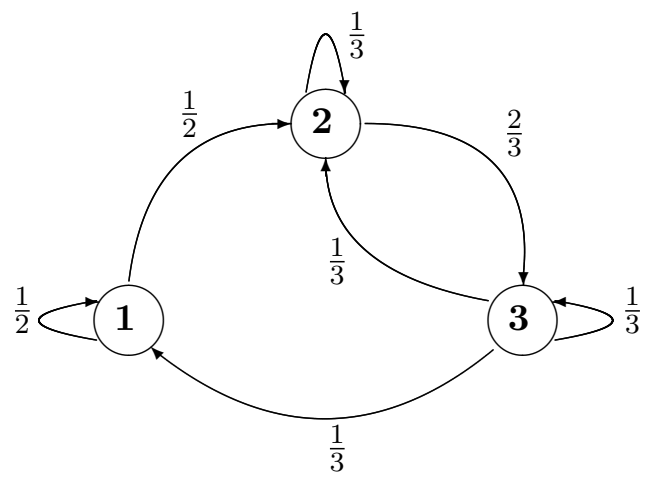

Como se puede observar, se tienen tres estados en donde todos ellos se comunican. La matriz de transición es

$$
\left(\begin{array}{ccc}
\frac{1}{2} & \frac{1}{2} & 0 \\
0 & \frac{1}{3} & \frac{2}{3} \\
\frac{1}{3} & \frac{1}{3} & \frac{1}{3}
\end{array}\right) .
$$

Teorema 11. Una cadena de Markov irreducible y positivo recurrente tiene una única distribución invariante $\mu$, dada por

$$
\mu(x)=\frac{1}{E\left(T_{x} \mid X_{0}=x\right)}, \quad x \in S .
$$

Demostración. Ver [21].

Teorema 12. (De Kolmogorov sobre condiciones de reversibilidad). Sea p irreducible. Una medida $\mu$ es reversible si y sólo si 
1. Si $p(x, y)>0$ entonces $p(y, x)>0$

2. Sea $n \geq 2$. Para alguna sucesión $x_{0}, x_{1}, \ldots, x_{n} \in S$ con $x_{n}=x_{0}$ y $\Pi_{1 \leq i \leq n} p\left(x_{i}, x_{i-1}\right)>0$ se tiene

$$
\Pi_{i=1}^{n} \frac{p\left(x_{i-1}, x_{i}\right)}{p\left(x_{i}, x_{i-1}\right)}=1
$$

Demostración. Probemos primero la necesidad. Supongamos que $\mu$ es una medida reversible. Si $p(x, y)>0$ entonces

$$
\begin{aligned}
p(y, x) & =P\left(X_{n}=x \mid X_{n-1}=y\right) \\
& =\frac{P\left(X_{n}=x, X_{n-1}=y\right)}{P\left(X_{n-1}=y\right)} \\
& =\frac{P\left(X_{n-1}=y, X_{n}=x\right)}{P\left(X_{n-1}=y\right)}
\end{aligned}
$$

Ya que $P\left(X_{n-1}=y\right) \neq 0$ tenemos $P\left(X_{n-1}=y\right)>0$ y puesto que $p(x, y)>0$ entonces $P\left(X_{n-1}=y, X_{n}=x\right)>0$ concluimos pues que $p(y, x)>0$.

Ya que $\mu$ está en balance detallado, entonces $p\left(x_{i}, x_{i-1}\right)=\frac{\mu\left(x_{i-1}\right)}{\mu\left(x_{i}\right)} p\left(x_{i-1}, x_{i}\right)$, así

$$
\begin{aligned}
\Pi_{i=1}^{n} \frac{p\left(x_{i-1}, x_{i}\right)}{p\left(x_{i}, x_{i-1}\right)} & =\frac{p\left(x_{0}, x_{1}\right) \ldots p\left(x_{n-1}, x_{n}\right)}{p\left(x_{1}, x_{0}\right) \ldots p\left(x_{n}, x_{n-1}\right)} \\
& =\frac{p\left(x_{0}, x_{1}\right) \ldots p\left(x_{n-1}, x_{n}\right)}{\frac{\mu\left(x_{0}\right)}{\mu\left(x_{1}\right)} p\left(x_{0}, x_{1}\right) \ldots \frac{\mu\left(x_{n-1}\right)}{\mu\left(x_{n}\right)} p\left(x_{n-1}, x_{n}\right)} \\
& =\frac{\mu\left(x_{1}\right) \mu\left(x_{2}\right) \ldots \mu\left(x_{n}\right)}{\mu\left(x_{0}\right) \mu\left(x_{1}\right) \ldots \mu\left(x_{n-1}\right)} \\
& =\frac{\mu\left(x_{n}\right)}{\mu\left(x_{0}\right)} \\
& =\frac{\mu\left(x_{0}\right)}{\mu\left(x_{0}\right)} \\
& =1
\end{aligned}
$$

Para la suficiencia sea $x \in S$ tal que existe $n \in \mathbb{N}$ y existen $x_{0}, x_{1}, \ldots, x_{n}$ con $x_{0}=a, x_{n}=x$ $y \prod_{i=1}^{n} p\left(x_{i-1}, x_{i}\right)>0$. Sea

$$
\mu(x)=\Pi_{i=1}^{n} \frac{p\left(x_{i-1}, x_{i}\right)}{p\left(x_{i}, x_{i-1}\right)}
$$

Sea $m \in \mathbb{N}, \exists y_{0}, y_{1}, \ldots, y_{m}$ con $y_{0}=a, y_{m}=x y \Pi_{i=1}^{n} p\left(y_{i-1}, y_{i}\right)>0$. 
Sean $z_{0}=x_{0}=a, z_{1}=x_{1}, \ldots, z_{n}=x_{n}=y_{m}=x, z_{n+1}=y_{m-1}, \ldots, z_{n+m}=y_{0}$. Observemos que $\Pi_{i=1}^{n+m} p\left(z_{i-1}, z_{i}\right)>0$. Entonces

$$
\Pi_{i=1}^{n+m} \frac{p\left(z_{i-1}, z_{i}\right)}{p\left(z_{i}, z_{i-1}\right)}=1
$$

pero

$$
\Pi_{i=1}^{n+m} \frac{p\left(z_{i-1}, z_{i}\right)}{p\left(z_{i}, z_{i-1}\right)}=\frac{\Pi_{i=1}^{n} p\left(x_{i-1}, x_{i}\right) \Pi_{j=1}^{m} p\left(y_{j}, y_{j-1}\right)}{\Pi_{i=1}^{n} p\left(x_{i}, x_{i-1}\right) \Pi_{j=1}^{m} p\left(y_{j-1}, y_{j}\right)}
$$

Sean $z_{0}=a_{0}=x_{0}, z_{1}=x_{1}, \ldots, z_{n}=x_{n}=x, z_{n+1}=y_{m}=y, \ldots, z_{n+m+1}=y_{0}$ y tenemos que

$$
\begin{gathered}
p\left(x_{i}, x_{i+1}\right)>0 \Rightarrow p\left(x_{i+1}, x_{i}\right)>0 \\
p\left(y_{i}, y_{i+1}\right)>0 \Rightarrow p\left(y_{i+1}, y_{i}\right)>0
\end{gathered}
$$

$y$ además $p(x, y)>0 \Rightarrow p(y, x)>0$. Ahora $\Pi_{i=1}^{n+m+1} p\left(z_{i-1}, z_{i}\right)>0$ y

$$
\Pi_{i=1}^{n+m+1} \frac{p\left(z_{i-1}, z_{i}\right)}{p\left(z_{i}, z_{i-1}\right)}=1
$$

Así, por lo anterior

$$
\Pi_{i=1}^{n+m} \frac{p\left(z_{i-1}, z_{i}\right)}{p\left(z_{i}, z_{i-1}\right)}=\frac{\Pi_{i=1}^{n} p\left(x_{i-1}, x_{i}\right) p(x, y) \Pi_{j=1}^{m} p\left(y_{i}, y_{i-1}\right)}{\Pi_{i=1}^{n} p\left(x_{i}, x_{i-1}\right) p(y, x) \Pi_{j=1}^{m} p\left(y_{i-1}, y_{i}\right)}=1
$$

pero

$$
\mu(x)=\frac{\prod_{i=1}^{n} p\left(x_{i-1}, x_{i}\right)}{\Pi_{i=1}^{n} p\left(x_{i}, x_{i-1}\right)}, \quad \mu(y)=\frac{\prod_{j=1}^{m} p\left(y_{i}, y_{i-1}\right)}{\prod_{j=1}^{m} p\left(y_{i-1}, y_{i}\right)}
$$

entonces

$$
\begin{aligned}
1 & =\frac{\Pi_{i=1}^{n} p\left(x_{i-1}, x_{i}\right) p(x, y) \Pi_{j=1}^{m} p\left(y_{i}, y_{i-1}\right)}{\Pi_{i=1}^{n} p\left(x_{i}, x_{i-1}\right) p(y, x) \Pi_{j=1}^{m} p\left(y_{i-1}, y_{i}\right)} \\
& =\mu(x) \frac{p(x, y)}{p(y, x)} \frac{1}{\mu(y)}
\end{aligned}
$$

Entonces de la última igualdad tenemos que

$$
\mu(x) p(x, y)=\mu(y) p(y, x)
$$

Para ilustrar esto consideremos el siguiente ejemplo: 
Ejemplo 9. Sea $S$ el espacio de estados tal que $S=\mathbb{N}$ y sean

$$
p(0, y)= \begin{cases}\frac{1}{2} & \text { si } y=0 \\ \frac{1}{2} & \text { si } y=1 \\ 0 & \text { si } y \geq 2\end{cases}
$$

$y \operatorname{para} x>0$

$$
p(x, y)=\left\{\begin{array}{cc}
\frac{1}{2} & \text { si } y=0 \\
\frac{1}{4} & \text { si } y=x \\
\frac{1}{4} & \text { si } y=x+1 \\
0 & \text { en otro caso }
\end{array}\right.
$$

las funciones de transición de la cadena. Entonces tenemos que la matriz de transición de la cadena es:

$$
\boldsymbol{P}=\left(\begin{array}{cccccc}
\frac{1}{2} & \frac{1}{2} & 0 & 0 & 0 & \ldots \\
\frac{1}{2} & \frac{1}{4} & \frac{1}{4} & 0 & 0 & \ldots \\
\frac{1}{2} & 0 & \frac{1}{4} & \frac{1}{4} & 0 & \ldots \\
\frac{1}{2} & 0 & 0 & \frac{1}{2} & \frac{1}{2} & \ldots \\
\vdots & \vdots & \vdots & \vdots & \vdots & \ddots
\end{array}\right)
$$

Con la definición de medida invariante $\mu(y)=\sum_{x} \mu(x) p(x, y)$ encontramos ésta con la matriz de transición anterior y obtenemos que $\mu=(\mu(0), \mu(1), \mu(2), \mu(3), \ldots)=\left(1 / 2,1 / 3,1 / 3^{2}, 1 / 3^{3}, \ldots\right)$. Sin embargo esta medida invariante no está en balance detallado pues por ejemplo:

$$
p(0,3) \mu(0)=0\left(\frac{1}{2}\right)=0 \neq \frac{1}{18}=\left(\frac{1}{2}\right)\left(\frac{1}{3^{2}}\right)=p(3,0) \mu(3) .
$$

Ejemplo 10. (Cadena con dos estados). Sea $T=\mathbb{N}$ y $\left\{X_{n}\right\}_{n \in T}$ una cadena de Markov con espacio de estados $S=\{0,1\}$. Sea $0 \leq p \leq 1$ y $0 \leq q \leq 1$ con matriz de transición

$$
\boldsymbol{P}=\left(\begin{array}{cc}
1-p & p \\
q & 1-q
\end{array}\right)
$$

Para este proceso tenemos que la distribución invariante es $\mu=\left(\frac{q}{q+p}, \frac{p}{q+p}\right)$ la cual es única y está en balance detallado. En el caso en que $p=q=0$ cualquier medida de probabilidad será distribución invariante. Entonces para una cadena de Markov con dos estados siempre tenemos balance detallado.

Ejemplo 11. (Cadena con tres estados). Sea $T=\mathbb{N}$ y $\left\{X_{n}\right\}_{n \in T}$ una cadena de Markov con espacio de estados $S=\{0,1,2\}$ donde su matriz de transición es

$$
\boldsymbol{P}=\left(\begin{array}{lll}
p(0,0) & p(0,1) & p(0,2) \\
p(1,0) & p(1,1) & p(1,2) \\
p(2,0) & p(2,1) & p(2,2)
\end{array}\right)
$$

con $\sum_{y} p(x, y)=1$ y $p(x, y) \geq 0$ para $x, y \in S$. Usando el teorema de Kolmogorov sobre condiciones de reversibilidad, la cadena $\left\{X_{n}\right\}_{n \in T}$ tendrá distribución invariante en balance detallado si y sólo si 
i) Si $p(x, y)>0$ entonces $p(y, x)>0$ con $x, y \in S$.

ii) Si $p(x, y) p(y, x)>0$ entonces $p(x, y) p(y, z) p(z, x)=p(y, x) p(z, y) p(x, z)$ con $x, y, z \in S$.

$$
\left(\begin{array}{lll}
p(0,0) & p(0,1) & p(0,2) \\
p(1,0) & p(1,1) & p(1,2) \\
p(2,0) & p(2,1) & p(2,2)
\end{array}\right)
$$

Ejemplo 12. Consideremos una cadena de Markov con espacio de estados $S=\{0,1,2\}$ donde su matriz de transición es

$$
\boldsymbol{P}=\left(\begin{array}{ccc}
0 & 1 & 0 \\
0 & 0 & 1 \\
1 & 0 & 0
\end{array}\right)
$$

Calculando su distribución invariante obtenemos que ésta es $\mu=\left(\frac{1}{3}, \frac{1}{3}, \frac{1}{3}\right)$. Pero la cadena no está en balance detallado pues

$$
\mu(1) p(1,0)=\frac{1}{3}(0) \neq \frac{1}{3}(1)=\mu(0) p(0,1) .
$$

\subsubsection{Cadena de Markov con espacio de estados finito}

Consideremos ahora una cadena de Markov $\left\{X_{t}\right\}_{t \in T}$ con espacio de estados finito $S=$ $\{1,2, \ldots, N\}$, tenemos el siguiente resultado dado por [13]. Sea $D=\left(d_{x y}\right)_{x y}=I-P$ con $P$ matriz de transición y sea $D(H)$ el determinante de $D$ con renglones y columnas en el conjunto de índices $H \subset S$ y $D(\emptyset)=1$.

Teorema 13. La única distribución de probabilidad invariante $\pi=\left(\pi_{x}\right)_{x \in S}$ de la cadena de Markov $\left\{X_{t}\right\}_{t \in T}$ puede ser expresada como

$$
\pi_{x}=\frac{D\left(\{x\}^{c}\right)}{\sum_{y \in S} D\left(\{y\}^{c}\right)}
$$

Demostración. La única distribución invariante $\pi$ es la solución del sistema de ecuaciones $\pi D=0$ y $\pi \mathbf{1}=1$ donde $\mathbf{1}=(1, \ldots, 1)^{t}$. Entonces debemos resolver

$$
\left(\pi_{1}, \ldots, \pi_{N}\right)\left(\begin{array}{cccc}
d_{11} & d_{12} & \ldots & d_{1 N} \\
d_{21} & d_{22} & \ldots & d_{2 N} \\
\vdots & \vdots & \ddots & \vdots \\
d_{N 1} & d_{N 2} & \ldots & d_{N N}
\end{array}\right)=\boldsymbol{O}
$$


$y \sum_{x=1}^{N} \pi_{x}=1$. Ya que $\sum_{y=1}^{N} d_{x y}=0$ entonces para $z \neq y$

$$
d_{x y}=-\sum_{z=1} d_{x z}
$$

Así basta resolver el sistema de ecuaciones

$$
\left(\pi_{1}, \ldots, \pi_{N}\right)\left(\begin{array}{cccccccc}
1 & d_{11} & d_{12} & \ldots & d_{1(y-1)} & d_{1(y+1)} & \ldots & d_{1 N} \\
1 & d_{21} & d_{22} & \ldots & d_{2(y-1)} & d_{2(y+1)} & \ldots & d_{2 N} \\
1 & \vdots & \vdots & \ddots & \vdots & \vdots & \ddots & \vdots \\
1 & d_{N 1} & d_{N 2} & \ldots & d_{N(y-1)} & d_{N(y+1)} & \ldots & d_{N N}
\end{array}\right)=\left(\begin{array}{c}
1 \\
0 \\
\vdots \\
0
\end{array}\right)
$$

con

$$
D_{y}=\left(\begin{array}{cccccccc}
1 & d_{11} & d_{12} & \ldots & d_{1(y-1)} & d_{1(y+1)} & \ldots & d_{1 N} \\
1 & d_{21} & d_{22} & \ldots & d_{2(y-1)} & d_{2(y+1)} & \ldots & d_{2 N} \\
1 & \vdots & \vdots & \ddots & \vdots & \vdots & \ddots & \vdots \\
1 & d_{N 1} & d_{N 2} & \ldots & d_{N(y-1)} & d_{N(y+1)} & \ldots & d_{N N}
\end{array}\right)
$$

entonces $\pi D_{y}=(1,0, \ldots, 0)$. Probemos que $\operatorname{det} D_{y} \neq 0$.

Sabemos que $D=I-P$ con $P$ matriz de transición la cual es irreducible y recurrente. Por el teorema de Perron-Frobenius tenemos que

$$
\operatorname{dim} \operatorname{ker}(I-P)=1
$$

entonces $N=\operatorname{dim} \operatorname{ker}(I-P)+\operatorname{dim} \operatorname{Im}(I-P)$ y además $\mathbf{1} \in \operatorname{ker}(I-P)$ pues $(I-P) \mathbf{1}=\boldsymbol{O}$. Supongamos que $\operatorname{dim} D_{y}=0$, entonces

$$
1=\sum_{l \neq y} B_{l} C_{l}
$$

pero los $C_{l}$ son linealmente independientes, por lo tanto $B_{l}=0$ lo cual es una contradicción por tanto concluimos que $\operatorname{dim} D_{y} \neq 0$.

Entonces tenemos que $\pi D_{y}=(1,0,0, \ldots, 0)$, es decir, $\pi=(1,0, \ldots, 0) D_{y}^{-1}$. Pero

$$
D_{y}^{-1}=\frac{\operatorname{adj}\left(D_{y}\right)}{\operatorname{det} D_{y}}
$$

con $\operatorname{adj}\left(D_{y}\right)$ matriz transpuesta de cofactores de $D_{y}$, es decir,

$$
D_{y}^{-1}=\frac{1}{\operatorname{det} D_{y}}\left(\begin{array}{cccc}
C F_{11} & C F_{21} & \ldots & C F_{N 1} \\
\vdots & \vdots & \ddots & \vdots \\
C F_{1 N} & C F_{2 N} & \ldots & C F_{N N}
\end{array}\right)^{T}
$$


entonces

$$
(1,0, \ldots, 0) D_{y}^{-1}=\frac{1}{\operatorname{det} D_{y}}\left[C F_{11}, C F_{21}, \ldots, C F_{N 1}\right]
$$

donde $C F_{x y}=(-1)^{y+1} D\left(\{y\}^{c}\right)$ por tanto

$$
\pi_{y}=\frac{D\left(\{y\}^{c}\right)}{(-1)^{y+1} \operatorname{det} D_{y}}
$$

Ahora, tenemos que

$$
\begin{aligned}
\operatorname{det} D_{y} & =\operatorname{det}\left(\begin{array}{cccccccc}
1 & d_{11} & d_{12} & \ldots & d_{1(y-1)} & d_{1(y+1)} & \ldots & d_{1 N} \\
1 & d_{21} & d_{22} & \ldots & d_{2(y-1)} & d_{2(y+1)} & \ldots & d_{2 N} \\
1 & \vdots & \vdots & \ddots & \vdots & \vdots & \ddots & \vdots \\
1 & d_{N 1} & d_{N 2} & \ldots & d_{N(y-1)} & d_{N(y+1)} & \ldots & d_{N N}
\end{array}\right) \\
& =\operatorname{det}\left(\begin{array}{ccccccccc}
1 & -d_{1 y} & d_{12} & \ldots & d_{1(y-1)} & d_{1(y+1)} & \ldots & d_{1 N} \\
1 & -d_{2 y} & d_{22} & \ldots & d_{2(y-1)} & d_{2(y+1)} & \ldots & d_{2 N} \\
1 & \vdots & \vdots & \ddots & \vdots & \vdots & \ddots & \vdots \\
1 & -d_{N y} & d_{N 2} & \ldots & d_{N(y-1)} & d_{N(y+1)} & \ldots & d_{N N}
\end{array}\right) \\
& =\left(\begin{array}{ccccccccc}
1 & d_{1 y} & d_{12} & \ldots & d_{1(j-1)} & d_{1(j+1)} & \ldots & d_{1 N} \\
1 & d_{2 y} & d_{22} & \ldots & d_{2(j-1)} & d_{2(j+1)} & \ldots & d_{2 N} \\
1 & \vdots & \vdots & \ddots & \vdots & \vdots & \ddots & \vdots \\
1 & d_{N y} & d_{N 2} & \ldots & d_{N(j-1)} & d_{N(j+1)} & \ldots & d_{N N}
\end{array}\right) \\
& =\left(\begin{array}{ccccccccc}
1 & d_{12} & d_{13} & \ldots & d_{1 y} & \ldots & d_{1 N} \\
1 & d_{22} & d_{23} & \ldots & d_{2 y} & \ldots & d_{2 N} \\
1 & \vdots & \vdots & \ddots & \vdots & \ddots & \vdots \\
1 & d_{N 2} & d_{N 3} & \ldots & d_{N y} & \ldots & d_{N N}
\end{array}\right) \\
& =(-1)^{y}(-1) \operatorname{det}(-1)^{y+1} \operatorname{det} D_{1} \\
&
\end{aligned}
$$

entonces $(-1)^{y+1} \operatorname{det} D_{j}=\operatorname{det} D_{1}$, ó bien $\pi_{y}(-1)^{y+1} \operatorname{det} D_{y}=\pi_{y} \operatorname{det} D_{1}$ y tomando la suma sobre $y$

$$
\begin{aligned}
\sum_{y \in S} \pi_{y}(-1)^{y+1} \operatorname{det} D_{y} & =\sum_{y \in S} \pi_{y} \operatorname{det} D_{1} \\
& =\operatorname{det} D_{1}
\end{aligned}
$$

Pero tenemos que $\pi_{y}(-1)^{y+1} \operatorname{det} D_{y}=D\left(\{y\}^{c}\right)$ por tanto $\operatorname{det} D_{1}=\sum_{y \in S} D\left(\{y\}^{c}\right)$, así

$$
\begin{aligned}
\pi_{y} & =\frac{D\left(\{y\}^{c}\right)}{(-1)^{y+1} \operatorname{det} D_{y}} \\
& =\frac{D\left(\{y\}^{c}\right)}{\operatorname{det} D_{1}} \\
& =\frac{D\left(\{y\}^{c}\right)}{\sum_{y \in S} D\left(\{y\}^{c}\right)}
\end{aligned}
$$




\subsubsection{Cadena de Markov con espacio de estados infinito numerable}

Ejemplo 13. (Línea de Espera). Una línea de espera con un servidor se puede modelar con un proceso estocástico en donde la variable aleatoria se define como el número de personas u objetos en el sistema en un momento dado con espacio de estados $S=\mathbb{N}$.

Una línea de espera es un cadena de Markov ya que la probabilidad condicional de llegar a un estado futuro depende solamente del estado actual en el que se encuentra el sistema, sin importar el estado inicial de dicho sistema. Sea $\xi_{n}$ el número de nuevos clientes que llegan durante el $n$-ésimo período. Suponemos que $\xi_{1}, \xi_{2}, \ldots$ son variables aleatorias independientes, no negativas y real valuadas y tienen en común la densidad $g$. Sea $X_{0}$ el número de clientes presentes inicialmente y para $n \leq 1, X_{n}$ denota el número de clientes presentes al final del $n$ ésimo período. Si $X_{n}=0$, entonces $X_{n+1}=\xi_{n+1}$ y si $X_{n} \leq 1$, entonces $X_{n+1}=X_{n}+\xi_{n+1}-1$. Así, la matriz de transición de una línea de espera está definida por

$$
W=\left(\begin{array}{ccccc}
g(0) & g(1) & g(2) & g(3) & \ldots \\
g(0) & g(1) & g(2) & g(3) & \ldots \\
0 & g(0) & g(1) & g(2) & \ldots \\
0 & 0 & g(0) & g(1) & \ldots \\
0 & 0 & 0 & g(0) & \ldots \\
\vdots & \vdots & \vdots & \vdots & \ddots
\end{array}\right)
$$

Como podemos observar en $W$, la línea de espera no está en balance detallado pues si $\pi$ es distribución invariante, se tiene que $0=\pi(2) \cdot 0=\pi(2) W(2,0) \neq \pi(0) W(0,2)=\pi(0) g(2)$. Sea $\mu=\sum_{x=0}^{\infty} x g(x)$ la esperanza. Las condiciones para que esta cadena sea irreducible y positivo recurrente son:

1. Si $g(0)>0$ y $g(0)+g(1)<1$, la línea de espera es irreducible.

2. Si la línea de espera es irreducible, entonces es positivo recurrente si $\mu<1$.

La prueba de estas condiciones se puede ver en [21]. Por lo tanto por el Teorema 11 existe su distribución invariante. Para calcularla usamos la función generadora como sigue: 
Sea $\mu$ la media, $\Psi(t)=\sum_{y=0}^{\infty} \pi(y) t^{y}$ con $\pi$ distribución invariante $y G(t)=\sum_{y=0}^{\infty} g(y) t^{y}$. Supongamos que $g(-x)=0$. Sea $f(w, x)=g\left(x-(w-1)^{+}\right)$y $\sum_{w=0}^{\infty} \pi(w) f(w, x)=\pi(x)$. Así

$$
\begin{aligned}
\Psi(t)=\sum_{y=0}^{\infty} \pi(y) t^{y} & =\sum_{y=0}^{\infty}\left[\sum_{w=0}^{\infty} \pi(w) f(w, y)\right] t^{y} \\
& =\sum_{w=0}^{\infty} \sum_{y=0}^{\infty} \pi(w) f(w, y) t^{y} \quad \text { donde los sumandos son no negativos } \\
& =\sum_{w=0}^{\infty} \pi(w) \sum_{y=0}^{\infty} f(w, y) t^{y} \\
& =\sum_{w=0}^{\infty} \pi(w) \sum_{y=0}^{\infty} g\left(y-(w-1)^{+}\right) t^{y} \\
& =\sum_{w=0}^{\infty} \pi(w) \sum_{y=0}^{\infty} g\left(y-(w-1)^{+}\right) t^{y-(w-1)^{+}} t^{(w-1)^{+}} \\
& =\sum_{w=0}^{\infty} \pi(w) t^{(w-1)^{+}} \sum_{y=0}^{\infty} g\left(y-(w-1)^{+}\right) t^{y-(w-1)^{+}} \\
& =\sum_{w=0}^{\infty} \pi(w) t^{(w-1)^{+}} G(t) \\
& =\left[\pi(0)+\pi(1)+\sum_{w=0}^{\infty} \pi(w) t^{w-1}\right] G(t) \\
& =\left[\pi(0)+\pi(1)+\frac{1}{t} \sum_{w=0}^{\infty} \pi(w) t^{w}\right] G(t) \\
& {\left[\pi(0)+\pi(1)+\frac{1}{t}(\Psi(t)-\pi(0)-\pi(1) t)\right] G(t) }
\end{aligned}
$$

Por lo tanto $\Psi(t)=\left[\frac{t-1}{t} \pi(0)+\frac{1}{t} \Psi(t)\right] G(t)$ y despejando a $\Psi(t)$ obtenemos que

$$
\Psi(t)=\frac{(t-1) G(t)}{t-G(t)} \Psi(0)
$$

Observemos que

$$
\lim _{t \rightarrow 1^{-}} \frac{t-1}{t-G(t)}=\lim _{t \rightarrow 1^{-}} \frac{1}{1-G^{\prime}(t)}
$$

pero $G^{\prime}(t)=\sum_{y=0}^{\infty} y t^{y} g(y)$ por lo tanto

$$
\lim _{t \rightarrow 1^{-}} G^{\prime}(t)=\sum_{y=0}^{\infty} y g(y)=\mu \quad y \quad \lim _{t \rightarrow 1^{-}} G(t)=1
$$


así

$$
\lim _{t \rightarrow 1^{-}} \frac{t-1}{t-G(t)}=\frac{1}{1-\mu}
$$

$y$

$$
\begin{aligned}
\lim _{t \rightarrow 1^{-}} \Psi(t)=\lim _{t \rightarrow 1^{-}} \frac{(t-1) G(t)}{1-G^{\prime}(t)} \pi(0) & =\frac{1}{1-\mu} \pi(0) \\
& =\frac{\pi(0)}{1-\mu}
\end{aligned}
$$

Donde este límite existe pues $\mu<1$. Por lo tanto, $\pi(0)=1-\mu y \frac{\Psi^{n}(0)}{n !}=\pi(n)$. Donde

$$
\frac{\Psi^{n}(t)}{\pi(0)}=\left(\frac{(t-1) G(t)}{t-G(t)}\right)^{(n)}=\sum_{k=0}^{n}\left(\begin{array}{c}
n \\
k
\end{array}\right)\left(\frac{t-1}{t-G(t)}\right)^{(n-k)} G^{k}(t) .
$$

Así, la distribución invariante de la línea de espera es:

$$
\pi(n)=(1-\mu) \sum_{k=0}^{n} \frac{1}{(n-k) ! k !}\left(\frac{t-1}{t-G(t)}\right)_{t=0}^{(n-k)} g(k)
$$

\subsubsection{Versión del Teorema Ergódico para cadenas de Markov}

Para cadenas de Markov con espacio de estados $S$ se tiene una versión del Teorema Ergódico el cual no es una consecuencia de éste.

Teorema 14. (Teorema Ergódico para Cadenas de Markov). Si $\left\{X_{n}\right\}_{n \in \mathbb{Z}}$ es irreducible, recurrente positiva y su distribución invariante es $\pi$, entonces para toda $f: S \longrightarrow \mathbb{R}$ tal que $\sum_{i \in S}|f(i)| \pi_{i}<\infty$, se cumple que, con probabilidad 1 ,

$$
\lim _{N \rightarrow \infty} \frac{1}{N} \sum_{k=1}^{N} f\left(X_{k}\right)=\sum_{i \in S} f(i) \pi_{i}
$$

Demostración. $\operatorname{Ver}[17] \mathrm{pg}$. 24.

Teorema 15. (Teorema de Convergencia). Sea p función de transición de una cadena de Markov irreducible y positivo recurrente. Si la cadena es aperiódica, es decir, el período de la cadena es uno. Entonces

$$
\lim _{n \rightarrow \infty} p^{n}(x, y)=\pi(y)
$$

para $x, y \in S$.

Demostración. Ver [2] pp. 262 - 263. 


\subsection{Proceso de Markov en tiempo continuo}

Sea $S$ el espacio de estados para los procesos de Markov en tiempo continuo un espacio métrico compacto y sea $T=\mathbb{R}_{+}$.

Definición 21. Sea $s, t \in T$. Un proceso estocástico $\left\{X_{t}\right\}_{t \in T}$ es un proceso de Markov con respecto a la filtración $\left\{\mathfrak{F}_{t}\right\}_{t \geq 0}$ si para cada $s<t$

$$
P\left(X_{t}=x \mid \mathfrak{F}_{s}\right)=P\left(X_{t}=x \mid X_{s}\right) .
$$

Sea $s \leq t$. Tenemos otra definición de proceso de Markov: Un proceso estocástico $\left\{X_{t}\right\}_{t \in T}$ es de Markov respecto a la filtración $\left\{\mathfrak{F}_{t}\right\}_{t \geq 0}$ si y sólo si para todo $t \in T$, para todo $A$ en $\mathfrak{F}_{t} \mathrm{y}$ para todo $B \in \sigma\left(\left\{X_{r}: t \leq r\right\}\right)$

$$
P\left[A \cap B \mid X_{t}\right]=P\left[A \mid X_{t}\right] P\left[B \mid X_{t}\right]
$$

es decir, con la información del pasado y del futuro, $A$ y $B$ son condicionalmente independientes. Ver [3] pp. 9 - 10.

\subsubsection{Construcción de un proceso de Markov}

Al proceso estocástico lo podemos pensar como $X: T \times \Omega \longrightarrow S^{T}$ donde $S^{T}$ es el conjunto $\{f: T \rightarrow S \mid f$ es función $\}$. Así, la distribución del proceso estocástico $\left\{X_{t}\right\}_{t \in T}$ es la distribución de $X$ tal que $P_{X}: \mathcal{C} \longrightarrow[0,1]$ y $P_{X}(A)=P(X \in A)$, con $A \in \mathcal{C}$.

Definición 22. Sea $(\Omega, \mathfrak{F}, P)$ espacio de probabilidad. Sea $\left\{X_{t}\right\}_{t \in T}$ un proceso con valores en $(S, \mathfrak{S})$. Sea $p$ una función de transición. Decimos que $\left\{X_{t}\right\}_{t \in T}$ está gobernado por $p$ si para alguna medida de probabilidad $\mu: \mathfrak{S} \rightarrow[0,1]$ se tiene que

$$
P\left[X\left(t_{n}\right) \in B_{n}, \ldots, X\left(t_{0}\right) \in B_{0}\right]=\int_{S} \mu_{0}\left(d x_{0}\right) \int_{B_{1}} p_{t_{1}}\left(x_{0}, d x_{1}\right), \ldots, \int_{B_{n}} p_{t_{n}-t_{n-1}}\left(x_{n-1}, d x_{n}\right)
$$

para todo $n \in \mathbb{N}, \forall 0 \leq t_{1} \leq \ldots \leq t_{n}$ y $B_{1}, B_{2}, \ldots, B_{n} \in \mathfrak{S}$.

Teorema 16. Si $\left\{X_{t}\right\}_{t \in T}$ está gobernado por una función de transición p, entonces $\left\{X_{t}\right\}_{t \in T}$ es de Markov respecto a la filtración $\left\{\mathfrak{F}_{t}\right\}_{t \geq 0}$.

Demostración. Ver [3], p. 183. 
El siguiente ejemplo muestra que una cadena de Markov es un proceso estacionario.

Ejemplo 14. Sea $\left\{X_{n}\right\}_{n \in \mathbb{Z}}$ una cadena de Markov con probabilidad de transición $p(x, A)$ $y$ distribución invariante $\pi$. Si $X_{0}$ tiene distribución $\pi$, entonces $X_{0}, X_{1}, \ldots$ es un proceso estacionario.

Esto es por que

$$
\begin{aligned}
P\left(\left\{\omega:\left(X_{0}, X_{1}, \ldots, X_{n}\right) \in B\right\}\right) & =\int_{B_{0}} \pi\left(\mathrm{d} x_{0}\right) \int_{B_{1}} p\left(x_{0}, \mathrm{~d} x_{1}\right) \ldots \int_{B_{n}} p\left(x_{n-1}, \mathrm{x}_{\mathrm{n}}\right) \\
& =\int_{B_{0}} \pi\left(\mathrm{d} x_{k}\right) \int_{B_{1}} p\left(x_{k}, \mathrm{~d} x_{k+1}\right) \ldots \int_{B_{n}} p\left(x_{k+n-1}, \mathrm{x}_{\mathrm{n}+\mathrm{k}}\right) \\
& =P\left(\left\{\omega:\left(X_{k}, X_{k+1}, \ldots, X_{n+k}\right) \in B\right\}\right)
\end{aligned}
$$

Ahora con el Teorema de Kolmogorov construyamos un proceso de Markov:

Dado $(S, \mathfrak{S})$ espacio medible. Dada $\mu: \mathfrak{S} \rightarrow[0,1]$ medida de probabilidad y dada $p:[0, \infty) \times$ $S \times \mathfrak{S} \rightarrow[0,1]$ función de transición con el Teorema de Kolmogorov construyamos un proceso.

Tenemos que $S^{\left\{t_{1}, \ldots, t_{n}\right\}}=S \times \ldots \times S=\left\{f:\left\{t_{1}, \ldots, t_{n}\right\} \rightarrow S\right\}$. Para todo $t \in \mathbb{R}_{+}$fijo, $S^{\{t\}}=\{f:\{t\} \rightarrow S\}$. Para $0 \leq t_{1} \leq \ldots \leq t_{n}$ consideremos el espacio $\left(S^{\left\{t_{1}, \ldots, t_{n}\right\}}, \mathfrak{S} \otimes \ldots \otimes \mathfrak{S}\right)$. Se puede probar con el Teorema de extensión de Carathéodory que existe una medida $\mu_{t}$ : $\mathfrak{S} \otimes \ldots \otimes \mathfrak{S} \rightarrow[0,1]$ tal que

$$
\mu_{t_{1} \ldots t_{n}}\left(B_{1} \times \ldots \times B_{n}\right)=\int_{S} \mu\left(d x_{0}\right) \int_{B_{1}} p_{t_{1}}\left(x_{0}, d x_{1}\right), \ldots, \int_{B_{n}} p_{t_{n}-t_{n-1}}\left(x_{n-1}, d x_{n}\right)
$$

Entonces sea $\theta=\left\{\mu_{t_{1} \ldots t_{n}}: n \in \mathbb{N}, 0 \leq t_{1} \leq \ldots \leq t_{n}\right\}$. Probaremos que $\theta$ es un sistema proyectivo. Tenemos que $\Pi: S^{\left\{t_{1}, \ldots, t_{n}\right\}} \longrightarrow S^{\left\{t_{1}, \ldots, t_{n-1}\right\}}$.

Por demostrar que $\mu_{t_{1} \ldots t_{n-1}}=\mu_{t_{1} \ldots t_{n}}\left(\Pi_{\left\{t_{1} \ldots t_{n-1}\right\}\left\{t_{1} \ldots t_{n}\right\}}^{-1}\right)$.

Por un lado tenemos que

$$
\mu_{t_{1} \ldots t_{n-1}}\left(B_{1} \times \ldots \times B_{n-1}\right)=\int_{S} \mu\left(d x_{0}\right) \int_{B_{1}} p_{t_{1}}\left(x_{0}, d x_{1}\right), \ldots, \int_{B_{n-1}} p_{t_{n-1}-t_{n-2}}\left(x_{n-2}, d x_{n-1}\right)
$$

y para $\mu_{t_{1} \ldots t_{n}}\left(\Pi_{\left\{t_{1} \ldots t_{n-1}\right\}\left\{t_{1} \ldots t_{n}\right\}}^{-1}\left(B_{1} \times \ldots \times B_{n-1}\right)\right)$ tenemos que

$$
\Pi_{\left\{t_{1} \ldots t_{n-1}\right\}\left\{t_{1} \ldots t_{n}\right\}}^{-1}\left(B_{1} \times \ldots \times B_{n-1}\right)=B_{1} \times \ldots \times B_{n-1} \times S
$$


entonces

$$
\begin{gathered}
\mu_{t_{1} \ldots t_{n}}\left(\Pi_{\left\{t_{1} \ldots t_{n-1}\right\}\left\{t_{1} \ldots t_{n}\right\}}^{-1}\left(B_{1} \times \ldots \times B_{n-1}\right)\right)= \\
\int_{S} \mu\left(d x_{0}\right) \int_{B_{1}} p_{t_{1}}\left(x_{0}, d x_{1}\right), \ldots, \int_{B_{n-1}} p_{t_{n-1}-t_{n-2}}\left(x_{n-2}, d x_{n-1}\right) \int_{S} p_{t_{n}-t_{n-1}}\left(x_{n-1}, d x_{n}\right)
\end{gathered}
$$

pero $\int_{S} p_{t_{n}-t_{n-1}}\left(x_{n-1}, d x_{n}\right)=p_{t_{n}-t_{n-1}}\left(x_{n-1}, S\right)=1$. Por tanto $\theta$ es un sistema proyectivo. Así consideraremos $\Omega=S^{\mathbb{R}_{+}}$y $\mathcal{C}=\sigma$-álgebra cilíndrica, $X_{t}: \omega \rightarrow S$ tal que $X_{t}(\omega)=\omega(t)$. Sea $P: \mathcal{C} \longrightarrow[0,1]$ el límite proyectivo.

\subsubsection{Semigrupo de Markov}

Definición 23. Sea $S$ un espacio métrico compacto con $\mathfrak{S}$ la $\sigma$-álgebra de Borel. Sea $C(S)$ el espacio de funciones continuas sobre $S$ con la norma del supremo, es decir, $\|f\|=\sup _{x \in S}|f(x)|$. Sea $\left\{\boldsymbol{T}_{t}\right\}_{t \geq 0}$ un semigrupo sobre $C(S)$ con $\boldsymbol{T}_{t} f \in C(S)$ para toda $t \geq 0$ y $f \in C(X)$. Decimos que $\left\{\boldsymbol{T}_{t}\right\}_{t \geq 0}$ es un semigrupo de Markov si satisface las siguientes propiedades:

1. $\boldsymbol{T}_{0}=I$.

2. $\boldsymbol{T}_{t+s} f=\boldsymbol{T}_{t} \boldsymbol{T}_{s} f \forall f \in C(S) y \forall s, t, \geq 0$.

3. $\boldsymbol{T}_{t} 1=1$ para todo $t$.

4. Para toda $f \in C(S)$ la función $t \mapsto \boldsymbol{T}_{t} f$ es continua por la derecha.

5. $\boldsymbol{T}_{t} f \geq 0$ para toda función no negativa $f \in C(S)$.

Lo que nos interesa estudiar es la familia de operadores de $\left\{T_{t}\right\}_{t \geq 0}$ definidos sobre $S$ por,

$$
T_{t} f(x)=\int_{S} \mathbf{T}_{t}(x, d y) f(y) .
$$

Esta familia está identificada con el semigrupo de Markov $\left\{\mathbf{T}_{t}\right\}$, ver [4] p.460. Ahora veamos que el semigrupo $\left\{T_{t}\right\}_{t \geq 0}$ definido en 3.1 efectivamente es un semigrupo de Markov.

Demostración. 1. Si $t=0$ entonces

$$
\begin{aligned}
T_{0} \mathbf{1}_{x} & =\int_{S} \boldsymbol{T}_{0}(x, d y) f \mathbf{1}_{y} \\
& =\int_{S} \delta_{x}(d y) \mathbf{1}_{y}=\mathbf{1}_{x}
\end{aligned}
$$


2. Para la condición de semigrupo se tiene que

$$
\begin{aligned}
T_{t}\left(T_{s} f(x)\right) & =\int_{S} \boldsymbol{T}_{t}(x, d y) T_{s} f(y) \\
& =\int_{S} \boldsymbol{T}_{t}(x, d y) \int_{S} \boldsymbol{T}_{s}(y, d z) f(z) \\
& =\int_{S}\left[\int_{S} \boldsymbol{T}_{t}(x, d y) \boldsymbol{T}_{s}(y, d z) f(z)\right] \\
& =\int_{S} \boldsymbol{T}_{t+s}(x, d y) f(y)=T_{t+s} f(x)
\end{aligned}
$$

3. $T_{t} \mathbf{1}_{x}=\int_{S} \boldsymbol{T}_{t}(x, d y) \mathbf{1}_{y}=\int_{S} \mathbf{1}_{y}(x)=\mathbf{1}_{x}$ para todat.

4. Para toda $f \in C(S)$ la función $t \mapsto \boldsymbol{T}_{t} f$ es continua por la derecha por ser semigrupo.

5. $T_{t} f(x)=\int_{S} \boldsymbol{T}_{t}(x, d y) f(y) \geq 0$ pues $\boldsymbol{T}_{t}(x, d y) \geq 0$.

Definición 24. El generador infinitesimal del semigrupo de Markov $\left\{T_{t}\right\}_{t \geq 0}$ es definido por

$$
L f=\lim _{t \downarrow 0} \frac{T_{t}-I}{t} f
$$

para $f \in D(f)=\left\{f \in C(S): \lim _{t \downarrow 0} \frac{T_{t}-I}{t} f\right.$ existe sobre $\left.C(S)\right\}$.

Ejemplo 15. (Modelo de Aprendizaje y Olvido). Para ilustrar la teoria anterior estudiamos un proceso estocástico de aprendizaje en ensayos de respuesta dicotómica y un número finito de estados absorbentes, en donde se construye un modelo probabilístico para representar un proceso de aprendizaje. El modelo incluye tanto el proceso de adquisición de información como el proceso de olvido de información, así como el supuesto de que el proceso puede terminar en uno cualquiera de $r$ estados. Definamos

- $A_{1}$ es el estado recurrente de adquisición.

- $A_{2}$ es el estado recurrente de olvido.

- $f_{i}, i=1,2, \ldots, r$ es el estado en que el individuo termina el proceso de aprendizaje, es un estado final y éste es absorbente.

- $(\tau, t), 0 \leq \tau \leq t \leq \infty$ es el intervalo temporal donde el sistema viaja en $A_{1}$ y $A_{2}$. 
Todas las transiciones entre los estados se supone que están gobernadas por la fuerza de las respuestas:

- $v_{A_{i} A_{j}}$ es la intensidad entre los estados $A_{i}$ y $A_{j}$ con $i \neq j$ y $i, j=1,2$.

- $\mu_{A_{j} f_{i}}, j=1,2, i=1,2, \ldots, r$ es la intensidad entre cualquiera de los estados $A$ y uno de los estados finales $f_{i}$.

- $v_{A_{i} A_{i}}=-\left(v_{A_{i} A_{j}}+\sum_{k=1}^{r} \mu_{A_{i} f_{k}}\right)$ es la intensidad de permanecer en el estado recurrente $A_{i}$ con $i \neq j$ y $i, j=1,2$.

Observemos que $v_{A_{1} A_{2}}>0$ así como $v_{A_{2} A_{1}}>0$ y $v_{A_{1} A_{1}} \neq 0$ pues si $v_{A_{1} A_{1}}=0$ entonces $A_{1}$ será absorbente. Se supone además que

$$
\sum_{i=1}^{r} \mu_{A_{1} f_{i}}>0 \text { o bien } \sum_{i=1}^{r} \mu_{A_{2} f_{i}}>0
$$

Entonces tenemos el espacio de estado $E=\left\{A_{1}, A_{2}, f_{1}, \ldots, f_{r}\right\}$ y notemos que el espacio de Banach $V$ en este ejemplo es $\mathbb{R}^{r+2}$, el cual es finito dimensional así definimos $L$ como el asociado al proceso:

$$
\boldsymbol{L}=\left(\begin{array}{cccccc}
v_{A_{1} A_{1}} & v_{A_{1} A_{2}} & \mu_{A_{1} f_{1}} & \mu_{A_{1} f_{2}} & \ldots & \mu_{A_{1} f_{r}} \\
v_{A_{2} A_{1}} & v_{A_{2} A_{2}} & \mu_{A_{2} f_{1}} & \mu_{A_{2} f_{2}} & \ldots & \mu_{A_{2} f_{r}} \\
0 & 0 & 0 & 0 & \ldots & 0 \\
0 & 0 & 0 & 0 & \ldots & 0 \\
\vdots & \vdots & \vdots & \vdots & \ddots & \vdots \\
0 & 0 & 0 & 0 & \ldots & 0
\end{array}\right)
$$

El polinomio característico es $P(\lambda)=\operatorname{det}(L-\lambda \boldsymbol{I})=(-\lambda)^{r}\left[\left(v_{A_{1} A_{1}}-\lambda\right)\left(v_{A_{2} A_{2}}-\lambda\right)-v_{A_{1} A_{2}} v_{A_{2} A_{1}}\right]$ donde los valores propios son $\lambda_{i}=0$ con $i=3,4, \ldots, r+2 y$

$$
\lambda_{1,2}=\frac{v_{A_{1} A_{1}}+v_{A_{2} A_{2}} \pm \sqrt{\left(v_{A_{1} A_{1}}+v_{A_{2} A_{2}}\right)^{2}-4\left(v_{A_{1} A_{1}} v_{A_{2} A_{2}}-v_{A_{1} A_{2}} v_{A_{2} A_{1}}\right)}}{2}
$$

donde $\lambda_{1}, \lambda_{2}<0$. Sea $K_{A}=\sqrt{\left(v_{A_{1} A_{1}}-v_{A_{2} A_{2}}\right)^{2}-4\left(v_{A_{1} A_{1}} v_{A_{2} A_{2}}-v_{A_{1} A_{2}} v_{A_{2} A_{1}}\right)}$ y sean

$$
s_{1}=\frac{v_{A_{1} A_{1}}-v_{A_{2} A_{2}}+K_{A}}{2} \quad y \quad s_{3}=\frac{v_{A_{1} A_{1}}-v_{A_{2} A_{2}}-K_{A}}{2}
$$

Los vectores propios asociados a los valores propios $\lambda_{1}$ y $\lambda_{2}$ son:

$$
\nu_{\lambda_{1}}=\left(1, \frac{-s_{1}}{v_{A_{1} A_{2}}}, 0, \ldots, 0\right)^{t}, \quad \nu_{\lambda_{2}}=\left(1, \frac{-s_{3}}{v_{A_{1} A_{2}}}, 0, \ldots, 0\right)^{t} .
$$


Para los otros $\lambda_{i}$ vectores con $i=3,4, \ldots, r+2$ consideramos el sistema

$$
\left(\begin{array}{cccccc}
v_{A_{1} A_{1}} & v_{A_{1} A_{2}} & \mu_{A_{1} f_{1}} & \mu_{A_{1} f_{2}} & \ldots & \mu_{A_{1} f_{r}} \\
v_{A_{2} A_{1}} & v_{A_{2} A_{2}} & \mu_{A_{2} f_{1}} & \mu_{A_{2} f_{2}} & \ldots & \mu_{A_{2} f_{r}} \\
0 & 0 & 0 & 0 & \ldots & 0 \\
0 & 0 & 0 & 0 & \ldots & 0 \\
\vdots & \vdots & \vdots & \vdots & \ddots & \vdots \\
0 & 0 & 0 & 0 & \ldots & 0
\end{array}\right)\left(\begin{array}{c}
m_{1} \\
m_{2} \\
m_{3} \\
m_{4} \\
\vdots \\
m_{r+2}
\end{array}\right)=\left(\begin{array}{c}
0 \\
0 \\
0 \\
0 \\
\vdots \\
0
\end{array}\right)
$$

donde el vector $\nu_{\lambda_{i}}=\left(x_{1 i}, x_{2 i}, 0, \ldots, 1, \ldots, 0\right)$ con $i=3, \ldots, r+2$ haciendo $m_{1}, m_{2}, m_{i} \neq 0$ y $m_{j}=0$ con $i=3, \ldots, r+2$ y $j \neq i$. Puesto que $v_{A_{1} A_{1}} v_{A_{2} A_{2}}-v_{A_{1} A_{2}} v_{A_{2} A_{1}} \neq 0$ con

$$
\begin{array}{ll}
x_{1 i}=\frac{\mu_{A_{2} f_{i-2}} v_{A_{1} A_{2}}-\mu_{A_{1} f_{i-2}} v_{A_{2} A_{2}}}{v_{A_{1} A_{1}} v_{A_{2} A_{2}}-v_{A_{1} A_{2}} v_{A_{2} A_{1}}}, & i=3,4, \ldots, r+2 \\
x_{2 i}=\frac{\mu_{A_{1} f_{i-2}} v_{A_{2} A_{2}}-\mu_{A_{2} f_{i-2}} v_{A_{1} A_{1}}}{v_{A_{1} A_{1}} v_{A_{2} A_{2}}-v_{A_{1} A_{2}} v_{A_{2} A_{1}}}, & i=3,4, \ldots, r+2
\end{array}
$$

Entonces la matriz de vectores propios es:

$$
\boldsymbol{V}=\left(\begin{array}{cc|cccc}
1 & 1 & x_{13} & x_{14} & \ldots & x_{1(r+2)} \\
\frac{-s_{1}}{v_{A_{1} A_{2}}} & \frac{-s_{3}}{v_{A_{1} A_{2}}} & x_{23} & x_{24} & \ldots & x_{2(r+2)} \\
\hline 0 & 0 & 1 & 0 & \ldots & 0 \\
0 & 0 & 0 & 1 & \ldots & 0 \\
\vdots & \vdots & \vdots & \vdots & \ddots & \vdots \\
0 & 0 & 0 & 0 & \ldots & 1
\end{array}\right)
$$

Consideremos los siguientes bloques de la matriz $\boldsymbol{V}$ :

$$
A=\left(\begin{array}{cc}
1 & 1 \\
\frac{-s_{1}}{v_{A_{1} A_{2}}} & \frac{-s_{3}}{v_{A_{1} A_{2}}}
\end{array}\right) \quad B=\left(\begin{array}{cccc}
x_{13} & x_{14} & \ldots & x_{1(r+2)} \\
x_{23} & x_{24} & \ldots & x_{2(r+2)}
\end{array}\right)
$$

Entonces podemos reescribir la matriz $\boldsymbol{V}$ de la siguiente forma:

$$
\boldsymbol{V}=\left(\begin{array}{cc}
A_{2 \times 2} & B_{2 \times r} \\
0_{r \times 2} & I_{r \times r}
\end{array}\right)
$$

con $0_{r \times 2}$ una matriz con entradas todas cero de dimensión $r$ renglones y 2 columnas, $I_{r \times r}$ es la matriz identidad de dimensión $r \times r$. Así, para obtener la matriz inversa de $\boldsymbol{V}$, trabajaremos con ella por bloques. Sea 


$$
\boldsymbol{U}=\boldsymbol{V}^{-1}=\left(\begin{array}{cc}
C_{2 \times 2} & D_{2 \times r} \\
E_{r \times 2} & F_{r \times r}
\end{array}\right)
$$

ya que $\boldsymbol{U}$ es inversa de $\boldsymbol{V}$, entonces

$$
I_{(r+2) \times(r+2)}=\left(\begin{array}{cc}
A_{2 \times 2} & B_{2 \times r} \\
0_{r \times 2} & I_{r \times r}
\end{array}\right)\left(\begin{array}{cc}
C_{2 \times 2} & D_{2 \times r} \\
E_{r \times 2} & F_{r \times r}
\end{array}\right)
$$

haciendo la multiplicación de matrices por bloques tenemos que:

$$
\left(\begin{array}{cc}
I_{2 \times 2} & 0_{2 \times r} \\
0_{r \times 2} & I_{r \times r}
\end{array}\right)=\left(\begin{array}{cc}
A C_{2 \times 2}+B E_{2 \times 2} & A D_{2 \times r}+B F_{2 \times r} \\
E_{r \times 2} & F_{r \times r}
\end{array}\right)
$$

Entonces $0=E, F=I, I=A C+B E \Rightarrow I=A C$ y $A D+B F=0 \Rightarrow A D+B=0$. Ya que $I=A C \rightarrow C=A^{-1}$, asi $A D=-B \Rightarrow D=-A^{-1} B$ y por tanto

$$
\boldsymbol{U}=\boldsymbol{V}^{-1}=\left(\begin{array}{cc}
A_{2 \times 2}^{-1} & -a^{-1} B_{2 \times r} \\
0_{r \times 2} & I_{r \times r}
\end{array}\right) \quad \text { donde } \quad A=\frac{v_{A_{1} A_{2}}}{s_{1}-s_{3}}\left(\begin{array}{cc}
\frac{-s_{3}}{v_{A_{1} A_{2}}} & -1 \\
\frac{s_{1}}{v_{A_{1} A_{2}}} & 1
\end{array}\right)
$$

Para lo cual, encontramos la matriz de transición de la cadena:

$$
P_{t}=\boldsymbol{V}\left(\begin{array}{cc|ccc}
e^{\lambda_{1} t} & 0 & 0 & \ldots & 0 \\
0 & e^{\lambda_{2} t} & 0 & \ldots & 0 \\
\hline 0 & 0 & 1 & \ldots & 0 \\
\vdots & \vdots & \vdots & \ddots & \vdots \\
0 & 0 & 0 & \ldots & 1
\end{array}\right)_{(r \times 2) \times(r \times 2)} \boldsymbol{U}
$$

si l es tal que: $l=\left(\begin{array}{cc}e^{\lambda_{1} t} & 0 \\ 0 & e^{\lambda_{2} t}\end{array}\right)$ podemos usar nuevamente operaciones de matrices por bloques:

$$
\begin{aligned}
P_{t} & =\left(\begin{array}{cc}
A & B \\
0 & I
\end{array}\right)\left(\begin{array}{cc}
l & 0 \\
0 & I
\end{array}\right)\left(\begin{array}{cc}
A^{-1} & -A^{-1} B \\
0 & I
\end{array}\right) \\
& =\left(\begin{array}{cc}
A l & B \\
0 & I
\end{array}\right)\left(\begin{array}{cc}
A^{-1} & -A^{-1} B \\
0 & I
\end{array}\right) \\
& =\left(\begin{array}{cc}
A l A^{-1} & -A l A^{-1} B+B \\
0 & I
\end{array}\right)
\end{aligned}
$$


donde todo se reduce a calcular $A l A^{-1}$ y $A l A^{-1} B+B$. Entonces

$$
\begin{aligned}
A l A^{-1} & =\left(\begin{array}{cc}
1 & 1 \\
\frac{-s_{1}}{v_{A_{1} A_{2}}} & \frac{-s_{3}}{v_{A_{1} A_{2}}}
\end{array}\right)\left(\begin{array}{cc}
e^{\lambda_{1} t} & 0 \\
0 & e^{\lambda_{2} t}
\end{array}\right)\left(\begin{array}{cc}
\frac{-s_{3}}{s_{1}-s_{3}} & \frac{-1}{s_{1}-s_{3}} \\
\frac{s_{1}}{s_{1}-s_{3}} & \frac{1}{s_{1}-s_{3}}
\end{array}\right) \\
& =\left(\begin{array}{cc}
\frac{s_{1} e^{\lambda_{2} t}-s_{3} e^{\lambda_{1} t}}{s_{1}-s_{3}} & \frac{v_{A_{1} A_{2}}\left(e^{\lambda_{2} t}-e^{\lambda_{1} t}\right)}{s_{1}-s_{3}} \\
\frac{v_{A_{2} A_{1}}\left(e^{\lambda_{2} t}-e^{\lambda_{1} t}\right)}{s_{1}-s_{3}} & \frac{s_{1} e^{\lambda_{1} t}-s_{3} e^{\lambda_{2} t}}{s_{1}-s_{3}}
\end{array}\right)
\end{aligned}
$$

para calcular $A l A^{-1} B+B$ tenemos que

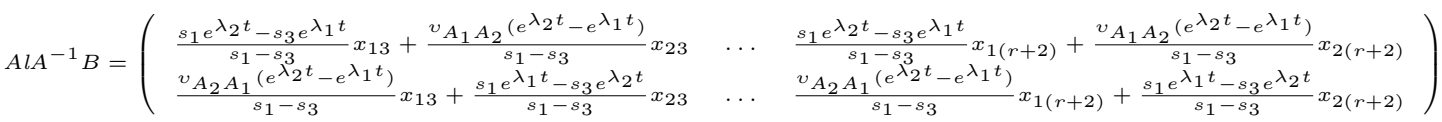
triz:

con $x_{1 i}, x_{2 i}$ definidos anteriormente, asi finalmente tenemos que $-A l A^{-1} B+B$ es la ma-

$$
\left(\begin{array}{ccc}
\frac{s_{1} e^{\lambda_{2} t}-s_{3} e^{\lambda_{1} t}}{s_{3}-s_{1}} x_{13}+\frac{v_{A_{1} A_{2}}\left(e^{\lambda_{2} t}-e^{\lambda_{1} t}\right)}{s_{3}-s_{1}} x_{23}+x_{13} & \ldots & \frac{s_{1} e^{\lambda_{2} t}-s_{3} e^{\lambda_{1} t}}{s_{3}-s_{1}} x_{1(r+2)}+\frac{v_{A_{1} A_{2}}\left(e^{\lambda_{2} t}-e^{\lambda_{1} t}\right)}{s_{3}-s_{1}} x_{2(r+2)}+x_{1(r+2)} \\
\frac{v_{A_{2} A_{1}}\left(e^{\lambda_{2} t}-e^{\lambda_{1} t}\right)}{s_{3}-s_{1}} x_{13}+\frac{s_{1} e^{\lambda_{1} t}-s_{3} e^{\lambda_{2} t}}{s_{3}-s_{1}} x_{23}+x_{23} & \ldots & \frac{v_{A_{2} A_{1}}\left(e^{\lambda_{2} t}-e^{\lambda_{1} t}\right)}{s_{3}-s_{1}} x_{1(r+2)}+\frac{s_{1} e^{\lambda_{1} t}-s_{3} e^{\lambda_{2} t}}{s_{3}-s_{1}} x_{2(r+2)}+x_{2((r+2))}
\end{array}\right)
$$

o bien, factorizando tenemos que $-A l A^{-1} B+B$ es:

$$
\left(\begin{array}{cccc}
\left(\frac{s_{1} e^{\lambda_{2} t}-s_{3} e^{\lambda_{1} t}}{s_{3}-s_{1}}+1\right) x_{13}+\frac{v_{A_{1} A_{2}}\left(e^{\lambda_{2} t}-e^{\lambda_{1} t}\right)}{s_{3}-s_{1}} x_{23} & \ldots & \left(\frac{s_{1} e^{\lambda_{2} t}-s_{3} e^{\lambda_{1} t}}{s_{3}-s_{1}}+1\right) x_{1(r+2)}+\frac{v_{A_{1} A_{2}}\left(e^{\lambda_{2} t}-e^{\lambda_{1} t}\right)}{s_{3}-s_{1}} x_{2(r+2)} \\
\frac{v_{A_{2} A_{1}}\left(e^{\lambda_{2} t}-e^{\lambda_{1} t}\right)}{s_{3}-s_{1}} x_{13}+\left(\frac{s_{1} e^{\lambda_{1}}-s_{3} e^{\lambda_{2} t}}{s_{3}-s_{1}}+1\right) x_{23} & \ldots & \frac{v_{A_{2} A_{1}}\left(e^{\lambda_{2} t}-e^{\lambda_{1} t}\right)}{s_{3}-s_{1}} x_{1(r+2)}+\left(\frac{s_{1} e^{\lambda_{1}} t-s_{3} e^{\lambda_{2} t}}{s_{3}-s_{1}}+1\right) x_{2(r+2)}
\end{array}\right)
$$

donde $s_{1}=v_{A_{1} A_{1}}-\lambda_{1}, s_{3}=v_{A_{1} A_{1}}-\lambda y s_{1}-s_{3}=\lambda_{2}-\lambda_{1}$, entonces ya que

$$
P_{t}=\left(\begin{array}{cc}
A l A^{-1} & -A l A^{-1} B+B \\
0 & I
\end{array}\right)
$$

donde $P_{t}$ es el semigrupo asociado al proceso y las probabilidades de transición entre estados de aprendizaje son:

$$
\begin{aligned}
& P_{A_{1} A_{1}}=\frac{e^{\lambda_{1} t}\left(\lambda_{2}-v_{A_{2} A_{2}}\right)}{\lambda_{2}-\lambda_{1}}+\frac{e^{\lambda_{2} t}\left(\lambda_{1}-v_{A_{2} A_{2}}\right)}{\lambda_{1}-\lambda_{2}}, \\
& P_{A_{2} A_{2}}=\frac{e^{\lambda_{1} t}\left(\lambda_{2}-v_{A_{1} A_{1}}\right)}{\lambda_{2}-\lambda_{1}}+\frac{e^{\lambda_{2} t}\left(\lambda_{1}-v_{A_{1} A_{1}}\right)}{\lambda_{1}-\lambda_{2}},
\end{aligned}
$$




$$
P_{A_{1} A_{2}}=\frac{e^{\lambda_{1} t} v_{A_{1} A_{2}}}{\lambda_{1}-\lambda_{2}}+\frac{e^{\lambda_{2} t} v_{A_{1} A_{2}}}{\lambda_{2}-\lambda_{1}} \quad y \quad P_{A_{2} A_{1}}=\frac{e^{\lambda_{1} t} v_{A_{2} A_{1}}}{\lambda_{2}-\lambda_{1}}+\frac{e^{\lambda_{2} t} v_{A_{2} A_{1}}}{\lambda_{1}-\lambda_{2}}
$$

Observemos que por la información que nos da la matriz de transición, las probabilidades de estar en un estado final y pasar a un estado recurrente $A_{1}$ y $A_{2}$ son cero, es decir,

$$
P_{f_{j} A_{i}}=0 \quad \text { para toda } i=1,2 \text { y } j=3,4, \ldots,(r+2)
$$

Las probabilidades de transición a un estado final son :

$$
\begin{aligned}
Q_{A_{1} f_{j}} & =\frac{e^{\lambda_{1} t}-1}{\lambda_{1}\left(\lambda_{1}-\lambda_{2}\right)}\left[\left(\lambda_{1}-v_{A_{2} A_{2}}\right) \mu_{A_{1} f_{j}}+v_{A_{1} A_{2}} \mu_{A_{2} f_{j}}\right] \\
Q_{A_{2} f_{j}} & =\frac{e^{\lambda_{2} t}-1}{\lambda_{2}\left(\lambda_{2}-\lambda_{1}\right)}\left[\left(\lambda_{2}-v_{A_{1} A_{1}}\right) \mu_{A_{2} f_{j}}+v_{A_{2} A_{1}} \mu_{A_{1} f_{j}}\right]
\end{aligned}
$$

para $j=1,2, \ldots, r$. Como lo muestra la matriz de transición, tenemos $r$ estados absorbentes, es decir,

$$
P_{f_{j} f_{j}}=1 \quad \text { donde } j=1,2, \ldots, r
$$

Sabemos que $\lambda_{1}, \lambda_{2}<0$, entonces pues tomemos las probabilidades de transición $P_{A_{i} A_{i}}$ con $i=1,2$.

$$
\begin{aligned}
\lim _{t \rightarrow 0} P_{A_{i} A_{i}} & =\frac{e^{\lambda_{1} t}\left(\lambda_{2}-v_{A_{i} A_{i}}\right)}{\lambda_{2}-\lambda_{1}}+\frac{e^{\lambda_{2} t}\left(\lambda_{1}-v_{A_{i} A_{i}}\right)}{\lambda_{1}-\lambda_{2}} \\
& =0
\end{aligned}
$$

para $P_{A_{i} A_{k}}$ con $i \neq k$ y $i, k=1,2$.

$$
\begin{aligned}
\lim _{t \rightarrow 0} P_{A_{i} A_{k}} & =\frac{e^{\lambda_{i} t} v_{A_{i} A_{k}}}{\lambda_{i}-\lambda_{k}}+\frac{e^{\lambda_{k} t} v_{A_{i} A_{k}}}{\lambda_{k}-\lambda_{i}} \\
& =0
\end{aligned}
$$

$Y$ finalmente para $Q_{A_{i} f_{j}}$ con $i, k=1,2 ., j=1,2, \ldots, r$ y $i \neq j \neq k$ tenemos:

$$
\begin{aligned}
\lim _{t \rightarrow 0} Q_{A_{i} f_{j}} & =\lim _{t \rightarrow 0} \frac{e^{\lambda_{i} t}-1}{\lambda_{i}\left(\lambda_{i}-\lambda_{k}\right)}\left[\left(\lambda_{i}-v_{A_{k} A_{k}}\right) \mu_{A_{i} f_{j}}+v_{A_{i} A_{k}} \mu_{A_{k} f_{j}}\right] \\
& =\frac{-1}{\lambda_{i}\left(\lambda_{i}-\lambda_{k}\right)}\left[\left(\lambda_{i}-v_{A_{k} A_{k}}\right) \mu_{A_{i} f_{j}}+v_{A_{i} A_{k}} \mu_{A_{k} f_{j}}\right]
\end{aligned}
$$

Es decir, la probabilidad de que el individuo transite en los estados absorbentes por un tiempo es cero, asi podemos asegurar que siempre se alcanza un estado final absorbente en cualquier momento. 


\subsection{Cadena de Markov encajada de un proceso de Markov de Saltos.}

Consideremos un proceso a tiempo continuo $\left\{X_{t}\right\}_{t \in T}$ con $T=\mathbb{R}_{+}$tal que comienza en el estado $x_{0}$ al tiempo $t=0$ y permanece en este estado un tiempo aleatorio $\tau_{1}$ hasta que salta al estado $x_{1}$ distinto del anterior y permanece un tiempo aleatorio $\tau_{2}$ hasta saltar a un nuevo estado $x_{3}$ y permanece en este estado un tiempo aleatorio $\tau_{3}$ y así sucesivamente como se muestra en la siguiente figura.

\section{Proceso de Saltos}

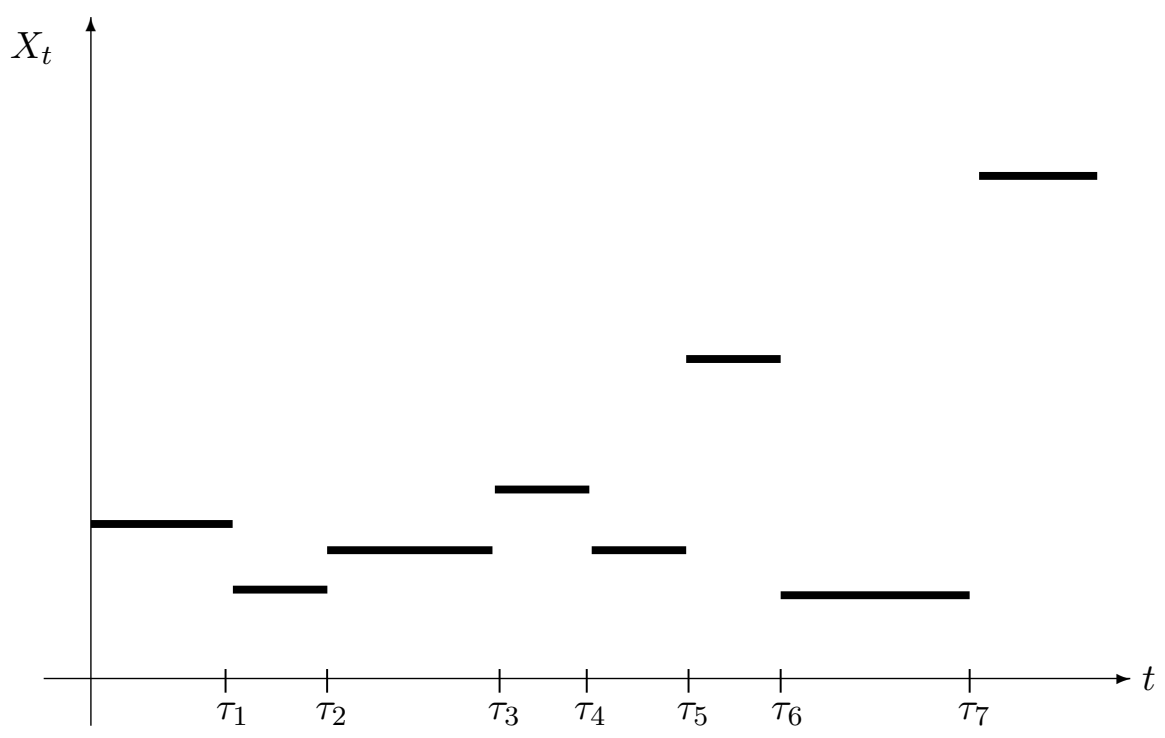

Definición 25. Sea $S$ un conjunto a lo más numerable. Sea $X_{t}$ es estado del sistema al tiempo $t$, definido por

$$
X_{t}=\left\{\begin{array}{cc}
Y_{0}, & \text { si } 0 \leq t<\tau_{1} \\
Y_{1}, & \text { si } \tau_{1} \leq t<\tau_{2} \\
Y_{2}, & \text { si } \tau_{2} \leq t<\tau_{3} \\
\vdots &
\end{array}\right.
$$

con $Y_{0}, Y_{1}, \ldots$ variables aleatorias con valores en $S$ y con $0 \leq \tau_{1}<\tau_{2}<\tau_{3}<\ldots$ donde $\tau_{1}, \tau_{2}, \tau_{3}, \ldots$ son variables aleatorias con valores en $T$. El proceso $\left\{X_{t}\right\}_{t \in T}$ es llamado proceso de Saltos.

Para un proceso definido anteriormente puede suceder que no esté definido para toda $t \in T$, esto pasa cuando los tiempos de estancia en los estados $X_{\tau_{i}}$ (es decir, la diferencia 
$\left.\gamma_{x}=\tau_{i}-\tau_{i-1}\right)$ sean cada vez más pequeños de tal forma que $\operatorname{lím}_{i \rightarrow \infty} \tau_{i}<\infty$, es decir, el proceso realiza un número infinito de saltos en un intervalo de tiempo acotado. Cuando esto sucede decimos que el proceso explota. Para evitar la explosión del proceso de saltos supondremos que $\lim _{i \rightarrow \infty} \tau_{i}=\infty$.

Supongamos que el proceso de Saltos $\left\{X_{t}\right\}_{t \in T}$ es un proceso de Markov. Así, el proceso $\left\{Y_{n}\right\}_{n \in \mathbb{N}}$ es una cadena de Markov la cual es llamada la cadena encajada del proceso de Markov $\left\{X_{t}\right\}_{t \in T}$, donde las variables $\tau_{i}-\tau_{i-1}$ son independientes y $P\left(\tau_{i}-\tau_{i-1}>t \mid Y_{i}=x\right)=q_{x} e^{-q_{x} t}$ con $Q$ matriz de transición de la cadena encajada. Para garantizar la existencia de un proceso de saltos seguiremos a [3].

Definición 26. (Lamperti). Sea $(S, d)$ espacio métrico y $\mathfrak{S}$ la $\sigma$-álgebra de Borel. Una función de transición tiene la propiedad de Feller sobre $S$ y $\mathfrak{S}$ si $T_{t} f$ definido por

$$
T_{t} f(x)=\int_{S} p(t, x, d y) f(y)
$$

es una función continua siempre que $f$ sea acotada y continua.

Definición 27. Sea $(S, d)$ espacio métrico y $\mathfrak{S}$ la $\sigma$-álgebra de Borel.

i) Una función de transición $p_{t}$ sobre $(S, \mathfrak{S})$ se dice que es continua estocásticamente si

$$
\lim _{t \rightarrow 0^{+}} p_{t}\left(x, N_{\epsilon}(x)\right)=1
$$

para toda $\epsilon>0, x \in S y$ con $N_{\epsilon}(x)=\{y \in S: d(x, y)<\epsilon\}$.

ii) Se dice que $p_{t}$ es uniformemente continua estocásticamente si el límite anterior converge uniformemente en $x$ para cualquier $\epsilon$ fijo positivo.

Definición 28. Una función de transición que es uniformemente continua estocásticamente y tiene la propiedad Feller sobre un espacio de estados $S$ métrico compacto es llamada una función de transición Normal.

Cabe mencionar que el término Normal no se refiere a la distribución Normal de una variable aleatoria.

Teorema 17. Sea $S$ un espacio métrico compacto y supongamos que $\left\{X_{t}\right\}_{t \in T}$ es un proceso estocástico con valores en $S$ el cual es gobernado por una función de transición de Markov Normal. Entonces existe un proceso $\left\{\widetilde{X}_{t}\right\}_{t \in T}$ modificación de $\left\{X_{t}\right\}_{t \in T}$, tal que excepto para un $\omega$-conjunto de probabilidad cero, las trayectorias $\widetilde{X}_{(\cdot)}(\omega)$ son continuas por la derecha y tienen limite por la izquierda $\forall t \in T$. 
Demostración. Ver [3], p. $18 \%$.

Ya que procesos uno modificación del otro tienen las mismas distribuciones finito dimensionales, $\left\{\widetilde{X}_{t}\right\}_{t \in T}$ es gobernado por la misma función de transición que $\left\{X_{t}\right\}_{t \in T}$ y tiene la misma distribución inicial. Sin embargo, que el espacio de estado $S$ sea métrico compacto es una hipótesis fuerte. Puesto que en este trabajo sólo utilizamos espacio de estados $S=\mathbb{R}^{n}$ ó $S=\mathbb{Z}^{n}$ con $n \geq 0$ los cuales son localmente compactos y son unión numerable de compactos podemos añadir un punto $\Delta \notin S$ al espacio $S$ para utilizar el siguiente resultado

Teorema 18. Sea $S$ un espacio topológico localmente compacto y $\tau$ su topología. Sea $\Delta$ un simbolo tal que $\Delta \notin S$. Sea

$$
\begin{aligned}
S_{\Delta} & :=S \cup\{\Delta\} \\
\tau_{\Delta} & :=\tau \cup\left\{A \subset S_{\Delta}: S \backslash A \text { compacto }\right\}
\end{aligned}
$$

entonces $\left(S_{\Delta}, \tau_{\Delta}\right)$ es un espacio topológico compacto haussdorff.

Demostración. Ver [15], p. 183.

La topología $\tau_{\Delta}$ es llamada la compactificación por un punto. Entonces todo espacio localmente compacto está contenido en un espacio compacto. Además si $S$ es un espacio métrico localmente compacto el cual es unión numerable de compactos entonces su compactificación por un punto $S_{\Delta}$ es metrizable. Así tendremos que $S_{\Delta}$ es un espacio de estados métrico compacto con la siguiente función de transición.

Teorema 19. Sea $S$ un espacio métrico localmente compacto y sea p una función de transición definida como $p:[0, \infty) \times S \times \mathfrak{B}(S) \longrightarrow[0,1]$. Entonces la función definida por $\widetilde{p}:[0, \infty) \times$ $S_{\Delta} \times \mathfrak{B}\left(S_{\Delta}\right) \longrightarrow[0,1]$ con

$$
\widetilde{p}(t, e, A)=\left\{\begin{array}{cc}
p(t, e, A \backslash\{\Delta\}) & \text { si } e \in S \\
\mathbf{1}_{A}(\Delta) & \text { si } e=\Delta
\end{array}\right.
$$

es función de transición.

Demostración. Sea $t \geq 0$ fijo. Consideremos dos casos: $e \in S$ y $e \notin S$.

- Si $e \in S$ entonces $e \neq \Delta$ por lo tanto

i) $\widetilde{p}(t, e, A)=p(t, e, A \backslash\{\Delta\}) \geq 0$. 
ii) Sean $A_{1}, A_{2}, \ldots \in \mathfrak{B}(S)$ con $A_{i} \cap A_{j}=0$ para $i \neq j$. Entonces

$$
\begin{aligned}
\widetilde{p}\left(t, e, \cup_{i} A_{i}\right) & =p\left(t, e, \cup_{i} A_{i} \backslash\{\Delta\}\right) \\
& =p\left(t, e, \cup_{i}\left(A_{i} \backslash\{\Delta\}\right)\right) \\
& =\sum_{i} p\left(t, e, A_{i} \backslash\{\Delta\}\right) \\
& =\sum_{i} \widetilde{p}\left(t, e, A_{i} \backslash\{\Delta\}\right)
\end{aligned}
$$

iii)

$$
\begin{aligned}
\widetilde{p}\left(t, e, S_{\Delta}\right) & =p\left(t, e, S_{\Delta} \backslash\{\Delta\}\right) \\
& =p(t, e, S) \\
& =1
\end{aligned}
$$

iv)

$$
\begin{aligned}
\int_{S_{\Delta}} \widetilde{p}(s, e, d y) \widetilde{p}(t, y, A) & =\int_{S_{\Delta}} p(s, e, d y) p(t, y, A \backslash\{\Delta\}) \\
& =\int_{S} p(s, e, d y) p(t, y, A \backslash\{\Delta\}) \\
& =p(t, e, A \backslash\{\Delta\}) \\
& =\widetilde{p}(t, e, A) \\
& =\widetilde{p}(t+s, e, A)
\end{aligned}
$$

- Si $e=\Delta$ entonces

i) $\widetilde{p}(t, \Delta, A)=\mathbf{1}_{A}(\Delta) \geq 0$.

ii) Sean $A_{1}, A_{2}, \ldots \in \mathfrak{B}(S)$, entonces

$$
\begin{aligned}
\widetilde{p}\left(t, \Delta, \cup_{i} A_{i}\right) & =\mathbf{1}_{\left(\cup_{i} A_{i}\right)}(\Delta) \\
& =\sum_{i} \mathbf{1}_{A_{i}}(\Delta) \\
& =\sum_{i} \widetilde{p}\left(t, \Delta, A_{i}\right)
\end{aligned}
$$

iii) $\tilde{p}\left(t, \Delta, S_{\Delta}\right)=\mathbf{1}_{S_{\Delta}}(\Delta)=1$. 
iv) Si $\Delta \in A$, entonces

$$
\begin{aligned}
\int_{S_{\Delta}} \widetilde{p}(s, \Delta, d y) \widetilde{p}(t, y, A) & =\int_{S_{\Delta}} \widetilde{p}(s, \Delta, d y) \\
& =\int_{S} \widetilde{p}(s, \Delta, d y) \\
& =1 \\
& =\widetilde{p}(t, \Delta, A) \\
& =\widetilde{p}(t+s, \Delta, A)
\end{aligned}
$$

Si $\Delta \notin A$, entonces

$$
\begin{aligned}
\int_{S_{\Delta}} \tilde{p}(s, \Delta, d y) \widetilde{p}(t, y, A) & =0 \\
& =\widetilde{p}(t, \Delta, A) \\
& =\widetilde{p}(t+s, \Delta, A)
\end{aligned}
$$

Para algún proceso continuo por la derecha sobre un espacio métrico los tiempos de permanencia

$$
\gamma_{x}=\inf \left\{t \geq 0: x_{t} \neq x\right\}
$$

son variables aleatorias, tomando ínf $\emptyset=+\infty$ sea $F_{x}(t)=P_{x}\left(\gamma_{x} \leq t\right)$.

Tenemos el siguiente lema, el cual se necesita para la prueba del teorema que afirma que las $\gamma_{x}$ tienen distribución exponencial con parámetro $q_{x}$.

Lema 1. Sea $a:[0, \infty] \rightarrow[0, \infty)$ y $f:[0, \infty) \rightarrow[0, \infty)$ tales que $\operatorname{lím}_{n \rightarrow \infty} a\left(\frac{t}{n}\right)^{n}=f(t)$ existe $\forall t \geq 0$, donde $f(t)$ es monótona. Entonces $f(t)=e^{-c t}$ para alguna constante $c \in[0, \infty]$.

Demostración. Para cualesquiera $\alpha \in \mathbb{N}$ y para todo $t \geq 0$ tenemos,

$$
\begin{aligned}
f(\alpha t) & =\lim _{n \rightarrow \infty} a\left(\frac{\alpha t}{n}\right)^{n} \\
& =\lim _{m \rightarrow \infty} a\left(\frac{\alpha t}{\alpha m}\right)^{\alpha m} \\
& =\left[\lim _{m \rightarrow \infty} a\left(\frac{t}{m}\right)^{m}\right]^{\alpha} \\
& =f(t)^{\alpha}
\end{aligned}
$$


Para $\alpha=\frac{1}{q} \operatorname{con} q \in \mathbb{N}$ y $q \geq 1$

$$
f(t)=f\left(\frac{q}{q} t\right)=\left(f\left(\frac{1}{q} t\right)\right)^{q}
$$

por lo tanto $f\left(\frac{1}{q} t\right)=(f(t))^{\frac{1}{q}}$. Así, si $\alpha=\frac{p}{q}$ entonces $f(\alpha t)=(f(t))^{\alpha}$ y ya que $f$ es monótona entonces tiene límites laterales en todo punto. La relación se cumple para todo $\alpha$. Y observemos que si $c=-\log f(1)$, entonces $f(t)=(f(1))^{t}=e^{-c t}$.

Teorema 20. Para cualesquier proceso continuo por la derecha gobernado por una función de transición de Markov $p_{t}$,

$$
F_{x}(t)=1-e^{-q_{x} t}
$$

con $t \geq 0$ y para algún $q_{x} \in[0, \infty]$. Es decir, Suponiendo que $Y_{i}=X_{\tau_{i}}=x, \gamma_{x}$ tiene distribución exponencial con parámetro $q_{x}$.

Demostración. Siguiendo a [3]. Consideremos

$$
f_{x}(t):=P_{x}\left(\gamma_{x} \geq t\right)=P_{x}\left(X_{s}=x, 0 \leq s<t\right)
$$

observemos que si $t_{1}<t_{2}$, entonces

$$
f_{x}\left(t_{1}\right)=P_{x}\left(\gamma_{x} \geq t_{1}\right)>P_{x}\left(\gamma_{x} \geq t_{2}\right)=f_{x}\left(t_{2}\right)
$$

por lo tanto $f_{x}$ es decreciente, es continua por la izquierda. Observemos que $F_{x}(t)=1-$ $P_{x}\left(\gamma_{x} \geq t\right)$ entonces $f_{x}(t)=e^{-q_{x} t}$.

Caso 1. Si $t=0$, entonces $f_{x}(0)=P_{x}\left(\tau_{x} \geq 0\right)=1$. Si $q_{x}=0$, entonces $x$ es una trampa, es decir, estado absorbente.

Caso 2. Si $\left\{X_{t}\right\}_{t \geq 0}$ no se queda en $x$ jamás, es decir, $x$ es instantáneo entonces $f_{x}(t)=$ $P_{x}\left(X_{s}=x, 0 \leq s<t\right)=0$ para todo $t>0$. Si $f_{x}(t)=0$ entonces $q_{x}=\infty$.

Ahora, sea $s \in\left\{0, \frac{t}{2^{n}}, \ldots, \frac{2^{n}-1}{2^{n}} t\right\}=D_{n}$ donde $D_{n} \subset D_{n+1}$. Sea $B_{n}^{t}=\cap_{s \in D_{n}}\left\{X_{s}=\right.$ $x\}, B_{n+1}^{t}=\cap_{s \in D_{n+1}}\left\{X_{s}=x\right\} \ldots y$ tenemos que $B_{n+1}^{t} \subset B_{n}^{t}$. Observemos que

$$
\lim _{n \rightarrow \infty} P\left(\cap_{s \in D_{n}}\left\{X_{s}=x\right\}\right)=\lim _{n \rightarrow \infty} P\left(X_{s}=x: s=\frac{i t}{2^{n}}, i=0, \ldots, 2^{n}-1\right)
$$


Entonces $\operatorname{lím}_{n \rightarrow \infty} P\left(B_{n}^{t}\right)$ existe y $\operatorname{lím}_{n \rightarrow \infty} P\left(B_{n}^{t}\right)=P\left(\cap_{n=1}^{\infty} B_{n}^{t}\right)$. Pero tenemos que por la continuidad por la derecha de las trayectorias de $\left\{X_{t}\right\}_{t \in T}$

$$
\begin{aligned}
\cap_{n=1}^{\infty} B_{n}^{t} & =\left\{\omega \in \Omega: \omega \in B_{n}^{t} \quad \forall n \in \mathbb{N}\right\} \\
& =\left\{\omega \in \Omega: X_{\frac{j t}{2^{n}}}=x, \forall n, \forall j=0,1, \ldots,\left(2^{n}-1\right)\right\} \\
& =\left\{\omega \in \Omega ; X_{r}(\omega)=x, \forall r \in[0, t]\right\}
\end{aligned}
$$

Así,

$$
\begin{aligned}
\lim _{n \rightarrow \infty} P\left(B_{n}^{t}\right) & =P\left(\left\{\omega \in \Omega: X_{r}(\omega)=x, \forall r \in[0, t]\right\}\right) \\
& =P\left(\left\{\tau_{x} \geq t\right\}\right) \\
& =f(t)
\end{aligned}
$$

Ahora, por la propiedad de Markov

$$
\begin{aligned}
P\left(B_{n}^{t}\right) & =P\left(\cap_{s \in D_{n}}\left\{X_{s}=x\right\}\right) \\
& =P\left(X_{0}=x, X_{\frac{t}{2^{n}}}=x, \ldots, X_{\frac{2^{n}-1}{2^{n}} t}=x\right) \\
& =P\left(X_{0}=x\right) P\left(X_{\frac{t}{2^{n}}}=x \mid X_{0}=x\right) P\left(x_{\frac{2 t}{2^{n}}}=x \mid X_{\frac{t}{2^{n}}}=x\right) P\left(X_{\frac{3 t}{2^{n}}}=x \mid X_{\frac{2 t}{2^{n}}}=x\right) \ldots \\
& =P\left(X_{0}=x\right) p\left(\frac{t}{2^{n}}, x,\{x\}\right) p\left(\frac{t}{2^{n}}, x,\{x\}\right) \ldots p\left(\frac{t}{2^{n}}, x,\{x\}\right) \\
& =P\left(X_{0}=x\right) p\left(\frac{t}{2^{n}}, x,\{x\}\right)^{2^{n}-1}
\end{aligned}
$$

Asi $f_{x}(t)=\lim _{n \rightarrow \infty}\left(B_{n}^{t}\right)=P\left(X_{0}=x\right) \lim _{n \rightarrow \infty} p\left(\frac{t}{2^{n}}, x,\{x\}\right)^{2^{n}-1}$. Y por el lema anterior tenemos que

$$
\begin{aligned}
f(t) & =P\left(X_{0}=x\right) \lim _{n \rightarrow \infty} p\left(\frac{t}{2^{n}}, x,\{x\}\right)^{2^{n}-1} \\
& =\lim _{n \rightarrow \infty} p\left(\frac{t}{2^{n}}, x,\{x\}\right)^{2^{n}-1} \\
& =e^{-q_{x} t}
\end{aligned}
$$

$\operatorname{con} q_{x} \in[0, \infty]$. Asi $F_{x}(t)=1-f(t)=1-e^{-q_{x} t}, t \geq 0$.

Entonces decimos que el proceso estocástico es un proceso de Saltos dado por la definición 25 con las siguientes características: 
- No explosión, es decir, $\operatorname{lím}_{n \rightarrow \infty} \tau_{n}=\infty$.

- La distribución condicional de $\gamma_{x}=\tau_{i}-\tau_{i-1}$, (dado $Y_{i}=x$ ) es exponencial con parámetro $q_{x}$.

- $Q_{x y}$ es la probabilidad de que la cadena salte del estado $x$ al estado $y$ con $Q_{x y} \geq 0$, $Q_{x x}=0$ y $\sum_{y} Q_{x y}=1$.

Definición 29. Sea $S$ a lo más numerable con la métrica discreta. Decimos que una función de transición $p(t, x, y)$ es conservativa con $t \in T$ si

$$
\sum_{y \in S} p(t, x, y)=1
$$

Proposición 3. Supongamos que $p(t, x, y)$ es conservativa. La función de transición $\widetilde{p}(t, e, A)$ tiene la propiedad de Feller si y sólo si $\operatorname{lím}_{x \rightarrow 0} p(t, x, y)=0$ para todo $t$ y $y$.

Demostración. Probemos primero la suficiencia. Observemos el siguiente resultado: $f$ : $\mathbb{N}_{\Delta} \longrightarrow \mathbb{R}$ es continua si y sólo si $\operatorname{lím}_{n \rightarrow \infty} f(n)=f(\infty)$. Tenemos que $x \longmapsto \mathbf{1}_{\{y\}}(x)$ tiene extensión continua en $\mathbb{N}_{\Delta} y$

$$
h(x)=\sum_{z \in \mathbb{N}_{\Delta}} \widetilde{p}(t, x, z) \mathbf{1}_{\{y\}}(z)=\sum_{z \in \mathbb{N}} \widetilde{p}(t, x, z) \mathbf{1}_{\{y\}}(z)=\widetilde{p}(t, x, y)
$$

$y$

$$
h(\infty)=\sum_{z \in \mathbb{N}_{\Delta}} \widetilde{p}(t, \infty, z) \mathbf{1}_{\{y\}}(z)=\sum_{z \in \mathbb{N}_{\Delta}} \delta_{\{\infty\}}(z) \mathbf{1}_{y}(z)=0
$$

Sea $f: \mathbb{N}_{\Delta} \longrightarrow \mathbb{R}$ continua. Hagamos $g(x)=\sum_{y \in \mathbb{N}_{\Delta}} \widetilde{p}(t, x, y) f(y)$. Mostremos que $\operatorname{lím}_{n \rightarrow \infty} g(n)$ existe. Sea $x \in \mathbb{N}$, entonces $g(x)=\sum_{y \in \mathbb{N}} p(t, x, y) f(x)$. Probemos que $g(x) \longrightarrow f(\infty)$ cuando $x \rightarrow \infty$ puesto que $g(\infty)=\sum_{y \in \mathbb{N}_{\Delta}} \widetilde{p}(t, \infty, y) f(y)=f(\infty)$. Entonces

$$
\begin{aligned}
|g(x)-f(\infty)| & \left.=\mid \sum_{y} p(t, x, y) f y j\right)-f(\infty) \mid \\
& =\left|\sum_{y} p(t, x, y)(f(y)-f(\infty))\right| \\
& \leq \sum_{y}|p(t, x, y) f(y)-f(\infty)| \\
& =\sum_{j}|p(t, x, y)||f(y)-f(\infty)|
\end{aligned}
$$

Puesto que $f$ es continua. Dado $\epsilon>0$ existe $y_{0} \in \mathbb{N}$ tal que $|f(y)-f(\infty)|<\frac{\epsilon}{2}$ para toda $y \geq y_{0}$. Sea $C=\sup \{f(x): x \in \mathbb{N}\}<\infty$, entonces existe $x_{0} \in \mathbb{N}$ tal que $p(t, x, y)<\frac{\epsilon}{2 C}$ para 
toda $x \geq x_{0}$, para toda $y \in\left\{0, \ldots, y_{0}\right\}$. Así

$$
\begin{aligned}
|g(x)-f(\infty)| & \leq \sum_{y=y_{0}+1}^{\infty} p(t, x, y)|f(y)-f(\infty)|+\sum_{y=0}^{y_{0}} p(t, x, y)|f(y)-f(\infty)| \\
& \leq \frac{\epsilon}{2}\left(\sum_{y=y_{0}+1}^{\infty} p(t, x, y)\right)+\frac{\epsilon}{2} \\
& \leq \frac{\epsilon}{2}+\frac{\epsilon}{2}=\epsilon
\end{aligned}
$$

Por lo tanto $\widetilde{p}(t, i, j)$ tiene la propiedad de Feller.

Para la necesidad tenemos que si $\widetilde{p}(t, x, y)$ tiene la propiedad de Feller, entonces

$$
\begin{gathered}
\lim _{x \rightarrow \infty} \sum_{y \in \mathbb{N}_{\Delta}} \widetilde{p}(t, x, y) \mathbf{1}_{\{z\}}(y)=\lim _{x \rightarrow \infty} \sum_{y \in \mathbb{N}} \widetilde{p}(t, x, y) \mathbf{1}_{\{z\}}(y) \text { existe. } \\
\text { pero } \quad \lim _{x \rightarrow \infty} \sum_{y \in \mathbb{N}_{\Delta}} \widetilde{p}(t, x, y) \mathbf{1}_{\{z\}}(y)=\lim _{x \rightarrow \infty} \widetilde{p}(t, x, z)=\lim _{x \rightarrow \infty} p(t, x, z)=\mathbf{1}_{\{z\}}(\infty)=0
\end{gathered}
$$

\subsubsection{Ecuación Integral hacia atrás de Kolmogorov}

Sea $S$ espacio de estados a lo más numerable, $q_{x}$ el parámetro de la distribución exponencial de los tiempos de estancia, $Q_{x y}$ la matriz de transición de la Cadena encajada de Salto y sea $p(t, x, y)$ una matriz de transición tal que

1. $p(0, x, y)=\delta_{x y} \operatorname{con} x, y \in S$.

2. $p(t, x, y) \geq 0$ con $t \in T$ y $x, y \in S$.

3. $\sum_{y} p(t, x, y) \leq 1$ con $t \in T$ y $x, y \in S$.

Y tenemos el siguiente resultado, 
Teorema 21. La función de transición $p(t, x, y)$ satisface la ecuación integral

$$
p(t, x, y)=\delta_{x y} e^{-q_{x} t}+\int_{0}^{t} q_{x} e^{-q_{x} s}\left(\sum_{z \neq x} Q_{x z} p(t-s, z, y)\right) d s, \quad t \in T .
$$

La ecuación 3.2 es llamada la ecuación integral hacia atrás de Kolmogorov

Demostración. Siguiendo a [21]. Si $x \in S$ es estado absorbente, entonces $p(t, x, y)=\delta_{x y}$ con $t \in T$ y se satisface la ecuación, por lo tanto supongamos que $x \in S$ es estado no absorbente. Entonces para un proceso que comienza en $x$, el evento $\left\{\tau_{1} \leq t, X_{\tau_{1}}=z\right.$ y $\left.X_{t}=y\right\}$ ocurre si $y$ sólo si el primer salto ocurre en algún tiempo $s \leq t$ y salta al estado $z$, y el proceso va de $z$ a $y$ en $t-s$ unidades de tiempo. Entonces

$$
P_{x}\left[\tau_{1} \leq t, X_{\tau_{1}}=z y X_{t}=y\right]=\int_{0}^{t} q_{x} e^{-q_{x} s} Q_{x z} p(t-s, z, y) d s
$$

y así

$$
\begin{aligned}
P_{x}\left[\left\{\tau_{1} \leq t, X_{t}=y\right\}\right] & =\sum_{z \neq x} P_{x}\left[\tau_{1} \leq t, X_{\tau_{1}}=z y X_{t}=y\right] \\
& =\sum_{z \neq x} \int_{0}^{t} q_{x} e^{-q_{x} s} Q_{x z} p(t-s, z, y) d s \\
& =\int_{0}^{t} q_{x} e^{-q_{x} s} \sum_{z \neq x} Q_{x z} p(t-s, z, y) d s
\end{aligned}
$$

Por otro lado tenemos que

$$
\begin{aligned}
P_{x}\left[\tau_{1}>t y X_{t}=y\right] & =\delta_{x y} P_{x}\left(\tau_{1}>t\right) \\
& =\delta_{x y} e^{-q_{x} t}
\end{aligned}
$$

por lo que

$$
\begin{aligned}
p(t, x, y) & =P_{x}\left(X_{t}=y\right) \\
& =P_{x}\left[\tau_{1}>t y X_{t}=y\right]+P_{x}\left[\tau_{1} \leq t y X_{t}=y\right] \\
& =\delta_{x y} e^{-q_{x} t}+\int_{0}^{t} q_{x} e^{-q_{x} s} \sum_{z \neq x} Q_{x z} p(t-s, z, y) d s
\end{aligned}
$$


Si reemplazamos $s$ por $t-s$ en la ecuación hacia atrás de Kolmogorov tenemos que

$$
p(t, x, y)=\delta_{x y} e^{-q_{x} t}+q_{x} e^{-q_{x} t} \int_{0}^{t} e^{q_{x} s}\left(\sum_{z \neq x} Q_{x z} p(s, z, y)\right) d s, \quad t \in T .
$$

la cual es continua en $t$ con $t \in T$, por lo tanto el integrando es continuo y podemos derivar respecto al tiempo, así

$$
\frac{\partial}{\partial t} p(t, x, y)=-q_{x} p(t, x, y)+q_{x} \sum_{z \neq x} Q_{x z} p(t, z, y), \quad t \in T .
$$

La ecuación (3.3) es llamada la ecuación diferencial hacia atrás de Kolmogorov. En particular, para $t=0$

$$
\frac{\partial}{\partial t} p(0, x, y)=-q_{x} \delta_{x y}+q_{x} Q_{x y} .
$$

Puesto que $p(t, x, y)$ con $x, y \in S$ es una sucesión de núcleos estocásticos, podemos calcular el generador infinitesimal de un proceso de Saltos el cual es

$$
\begin{aligned}
L(x, y) & =\left.\frac{\partial}{\partial t} p(t, x, y)(f(x))\right|_{t=0} \\
& =\sum_{y}\left[-q_{x} \delta_{x y}+q_{x} Q_{x y}\right] f(y) \\
& =-q_{x} \sum_{y} \delta_{x y} f(y)+q_{x} \sum_{y} Q_{x y} f(y) \\
& =q_{x}\left[\sum_{y} Q_{x y} f(y)-f(x)\right]
\end{aligned}
$$

Así, en forma matricial para todo $x \in S$ el generador infinitesimal de cualquier proceso de saltos se define como:

$$
L=q(Q-I)
$$

donde $q$ es la matriz diagonal con los elementos de la diagonal $q_{1}, q_{2}, \ldots, Q$ la matriz encajada y $I$ matriz identidad de la misma dimensión que $Q$. Existe un procedimiento iterativo para resolver la ecuación integral hacia atrás de Kolmogorov.

Teorema 22. Sean $x, y \in S$ fijos.

- Sea $p^{(0)}(x, y)=\delta_{x y} e^{-q_{x} t}$.

- Definiendo a $p^{n}(t, x, y)$ y $t \in T$, sea

$$
\begin{aligned}
p^{(n+1)}(t, x, y) & =\delta_{x y} e^{-q_{x} t}+q_{x} e^{-q_{x} t} \int_{0}^{t} e^{q_{x} s}\left(\sum_{z \neq x} Q_{x z} p^{(n)}(s, z, y)\right) d s \\
& =p^{(0)}(t, x, z)+q_{x} e^{-q_{x} t} \int_{0}^{t} e^{q_{x} s}\left(\sum_{z \neq x} Q_{x z} p^{(n)}(s, z, y)\right) d s
\end{aligned}
$$


3.3. CADENA DE MARKOV ENCAJADA DE UN PROCESO DE MARKOV DE SALTOS.53

Demostración. Mostraremos primero que $\operatorname{lím}_{n \rightarrow \infty} p^{(n)}(t, x, y)$ existe. Ahora observemos que

$$
0 \leq p^{(0)}(t, x, y) \leq 1
$$

y además

$$
p^{(1)}(t, x, y)=p^{(0)}(t, x, y)+q_{x} e^{-q_{x} t} \int_{0}^{t} e^{q_{x} s}\left(\sum_{z \neq x} Q_{x z} p^{(0)}(s, z, y)\right) d s
$$

pero $q_{x} e^{-q_{x} t} \int_{0}^{t} e^{q_{x} s}\left(\sum_{z \neq x} Q_{x z} p^{(0)}(s, z, y)\right) d s \geq 0$ por lo tanto

$$
p^{(1)}(t, x, y) \geq p^{(0)}(t, x, y)
$$

Ahora, puesto que $p^{(0)}(t, z, y)=1$, entonces

$$
\begin{aligned}
p^{(1)}(t, x, y) & =\delta_{x y} e^{-q_{x} t}+q_{x} e^{-q_{x} t} \int_{0}^{t} e^{q_{x} s} \sum_{z \neq x} Q_{x z} p^{(0)}(t, z, y) d s \\
& \leq \delta_{x y} e^{-q_{x} t}+q_{x} e^{-q_{x} t} \int_{0}^{t} e^{q_{x} s} d s \\
& =\delta_{x y} e^{-q_{x} t}+q_{x} e^{-q_{x} t}\left(\frac{1}{q_{x}}\right)\left[e^{q_{x} t}-1\right] \\
& =\delta_{x y} e^{-q_{x} t}+1-e^{q_{x} t} \\
& =1+e^{-q_{x} t}\left(\delta_{x y}-1\right) \leq 1
\end{aligned}
$$

$y$ por tanto

$$
0 \leq p^{0}(t, x, y) \leq p^{(1)}(t, x, y) \leq 1
$$

Supongamos que $0 \leq p^{(n)}(t, x, y) \leq p^{(n+1)}(t, x, y) \leq 1$. Mostraremos que $0 \leq p^{(n+1)}(t, x, y) \leq$ $p^{(n+2)}(t, x, y) \leq 1$. Tenemos que por hipótesis $p^{(n+1)}(t, x, y) \geq 0$ y dado que $p^{(n+1)}(t, x, y) \leq 1$ entonces $p^{(n+2)}(t, x, y)=1$ y por tanto $p^{(n+2)}(t, x, y) \leq 1$. Ahora, puesto que

$$
p^{(n)}(t, x, y) \leq p^{(n+1)}(t, x, y)
$$

entonces

$$
q_{x} e^{-q_{x} t} \int_{0}^{t} e^{q_{x} s} \sum_{z \neq x} Q_{x z} p^{(n)}(s, z, y) d s \leq q_{x} e^{-q_{x} t} \int_{0}^{t} e^{q_{x} s} \sum_{z \neq x} Q_{x z} p^{(n+1)}(s, z, y) d s
$$

$y$ consecuentemente

$\delta_{x y} e^{-q_{x} t} q_{x} e^{-q_{x} t} \int_{0}^{t} e^{q_{x} s} \sum_{z \neq x} Q_{x z} p^{(n)}(s, z, y) d s \leq \delta_{x y} e^{-q_{x} t} q_{x} e^{-q_{x} t} \int_{0}^{t} e^{q_{x} s} \sum_{z \neq x} Q_{x z} p^{(n+1)}(s, z, y) d s$

es decir,

$$
p^{(n+1)}(t, x, y) \leq p^{(n+2)}(t, x, y)
$$


y finalmente

$$
0 \leq p^{(n+1)}(t, x, y) \leq p^{(n+2)}(t, x, y) \leq 1
$$

Si $p(t, x, y)=\lim _{n \rightarrow \infty} p^{n}(t, x, y)$ es claro que $p(t, x, y)=\lim _{n \rightarrow \infty} p^{n}(t, x, y)$ es solución de la ecuación hacia atrás de Kolmogorov. Por tanto, por el teorema de convergencia monótona concluimos lo pedido

$$
p(t, x, y)=\lim _{n \rightarrow \infty} p^{(n)}(t, x, y)
$$

Tenemos un par de resultados importantes respecto a la solución de la ecuación integral hacia atrás de Kolmogorov los probamos siguiendo a [19].

Teorema 23. Sea $r(t, x, y)$ otra solución de la ecuación integral hacia atrás de Kolmogorov.

a) Entonces $0 \leq p(t, x, y) \leq r(t, x, y)$.

b) Si $p(t, x, y)$ es conservativa y $0 \leq \sum_{j} r(t, x, y) \leq 1$. Entonces $p(t, x, y)$ es la única solución no negativa, con $p(t, x, y) \leq 1$ de la ecuación integral hacia atrás de Kolmogorov.

Demostración. a) Sabemos que

$$
\begin{aligned}
r(t, x, y) & =p^{(0)}(t, x, y)+q_{x} e^{-q_{x} t} \int_{0}^{t} e^{q_{x} s} \sum_{z \neq x} Q_{x z} r(s, z, y) d s \\
& \geq p^{(0)}(t, x, y)
\end{aligned}
$$

Por lo tanto $0 \leq p^{(0)}(t, x, y) \leq r(t, x, y)$. Ahora supongamos que $0 \leq p^{(n)}(t, x, y) \leq r(t, x, y)$, entonces

$$
\begin{aligned}
0 \leq p^{(n+1)}(t, x, y) & =\delta_{x y} e^{-q_{x} t}+q_{x} e^{-q_{x} t} \int_{0}^{t} e^{q_{x} s} \sum_{z \neq x} Q_{x z} p^{(n)}(s, z, y) d s \\
& \leq \delta_{x y} e^{-q_{x} t}+q_{x} e^{-q_{x} t} \int_{0}^{t} e^{q_{x} s} \sum_{z \neq x} Q_{x z} r(s, z, y) d s=r(t, x, y) \\
& Y p(t, x, y)=\lim _{n \rightarrow \infty} p^{(n)}(t, x, y) \leq r(t, x, y) .
\end{aligned}
$$

b) Basta con probar que $\sum_{y} r(t, x, y)=1$ y puesto que $p(t, x, y) \leq r(t, x, y)$ entonces

$$
p(t, x, y)=r(t, x, y) \text {. }
$$


Sabemos que

$$
\begin{aligned}
p\left(t, x, y_{0}\right) \leq r\left(t, x, y_{0}\right) & =\sum_{y} r(t, x, y)-\sum_{y} r(t, x, y)+r\left(t, x, y_{0}\right) \\
& \leq 1-\sum_{y \neq y_{0}} r(t, x, y) \\
& \leq 1-\sum_{y \neq y_{0}} p(t, x, y) \\
& =\sum_{y} p(t, x, y)-\sum_{y \neq y_{0}} p(t, x, y) \\
& =p\left(t, x, y_{0}\right)
\end{aligned}
$$

Entonces

$$
p\left(t, x, y_{0}\right)=r\left(t, x, y_{0}\right) \quad y \quad \sum_{y \neq y_{0}} p(t, x, y)=\sum_{y \neq y_{0}} r(t, x, y)
$$

por tanto $\sum_{y} r(t, x, y)=1$.

Entonces la solución $p(t, x, y)$ es la mínima solución a la ecuación integral hacia atrás de Kolmogorov y ésta es llamada la solución minimal.

Teorema 24. La solución minimal $\{p(t, x, y)\}_{t \in T}$, cumple con la ecuación de ChapmanKolmogorov.

Demostración. Sea $W^{(0)}(t, x, y)=\delta_{x y} e^{-q_{x} t}=p^{(n)}(t, x, y)$ y sea

$$
W^{(n+1)}(t, x, y)=\int_{0}^{t} q_{x} e^{-q_{x} s} \sum_{z \neq x} Q_{x z} W^{(n)}(t-s, z, y) d s
$$


entonces $p(t, x, y)=\sum_{n=0}^{\infty} W^{(n)}(t, x, y)$ pues

$$
\begin{aligned}
p(t, x, y) & =\sum_{n=0}^{\infty} W^{(n)}(t, x, y) \\
& =W^{(0)}(t, x, y)+\sum_{n=1}^{\infty} W^{(n)}(t, x, y) \\
& =\delta_{x y} e^{-q_{x} t}+\sum_{n=1}^{\infty} \int_{0}^{t} q_{x} e^{-q_{x} s} \sum_{z \neq x} Q_{x z} W^{(n-1)}(t-s, z, y) d s \\
& =\delta_{x y} e^{-q_{x} t}+\int_{0}^{t} q_{x} e^{-q_{x} s} \sum_{z \neq x} Q_{x z} \sum_{n-1=0}^{\infty} W^{(n-1)}(t-s, z, y) d s \\
& =\delta_{x y} e^{-q_{x} t}+\int_{0}^{t} q_{x} e^{-q_{x} s} \sum_{z \neq x} Q_{x z} p(t-s, z, y) d s
\end{aligned}
$$

queremos mostrar que $p(t+s, x, y)=\sum_{z} p(s, x, z) p(t, z, y)$. Pero basta mostrar que

$$
W^{(n)}(t+s, x, y)=\sum_{m=0}^{n} \sum_{z} W^{(m)}(s, x, z) W^{(n-m)}(t, z, y) .
$$

Pues si lo anterior se cumple, entonces

$$
\begin{aligned}
p(t+s, x, y) & =\sum_{n=0}^{\infty} W^{(n)}(t+s, x, y) \\
& =\sum_{n=0}^{\infty}\left[\sum_{m=0}^{n} \sum_{z} W^{(m)}(s, x, z) W^{(n-m)}(t, z, y)\right] \\
& =\sum_{m=0}^{\infty}\left[\sum_{n=m}^{\infty} \sum_{z} W^{(m)}(s, x, z) W^{(n-m)}(t, z, y)\right] \\
& =\sum_{m=0}^{\infty} \sum_{z} W^{(m)}(s, x, z) \sum_{n=m}^{\infty} W^{(n-m)}(t, z, y) \\
& =\sum_{k} p(s, x, z) p(t, z, y)
\end{aligned}
$$

y entoces se obtine lo deseado. Ahora probemos (2.6). Si $n=0$, entonces

$$
\begin{aligned}
W^{(0)}(t+s, x, y) & =\delta_{x y} e^{-q_{x}(t+s)} \\
& =\delta_{x y} e^{-q_{x} t-q_{x} s} \\
& =\delta_{x y} e^{-q_{x} t} \delta_{x y} e^{-q_{x} s} \\
& =\sum_{z} W^{(0)}(s, x, z) W^{(0)}(t, z, y)
\end{aligned}
$$




\subsection{CADENA DE MARKOV ENCAJADA DE UN PROCESO DE MARKOV DE SALTOS.57}

Supongamos ahora que se cumple para algún n, entonces tenemos que:

$$
\begin{aligned}
& \sum_{m=0}^{n+1} \sum_{z} W^{(m)}(s, x, z) W^{(n+1-m)}(t, z, y)= \\
= & \sum_{k} W^{(0)}(s, x, z) W^{(n+1)}(t, z, y)+\sum_{m=1}^{n+1} \sum_{z} W^{(z)}(s, x, z) W^{(n+1-m)}(t, z, y) \\
= & \sum_{z} W^{(0)}(s, x, z) W^{(n+1)}(t, z, y)+\sum_{m=1}^{n+1} \sum_{z} \int_{0}^{s} q_{x} e^{-q_{x} u} \sum_{w \neq x} Q_{x w} W^{(m-1)}(s-u, w, z) W^{(n+1-m)}(t, z, y) d u \\
= & \sum_{z} \delta_{x z} e^{-q_{x} s} W^{(n+1)}(t, z, y)+\int_{0}^{s} q_{x} e^{-q_{x} s} \sum_{w \neq x} Q_{x w} \sum_{m=1}^{n+1} \sum_{z} W^{(m-1)}(s-u, w, z) W^{(n+1-m)}(t, z, y) d u \\
= & e^{-q_{x} s} W^{(n+1)}(t, x, y)+\int_{0}^{s} q_{x} e^{-q_{x} u} \sum_{w \neq x} Q_{x w} W^{(n)}(t+s, w, y) d u \\
= & e^{-q_{x} s} \int_{0}^{t} q_{x} e^{-q_{x} u} \sum_{w \neq x} Q_{x w} W^{(n)}(t-u, w, y)+\int_{0}^{s} q_{x} e^{-q_{x} u} \sum_{w \neq x} Q_{x w} W^{(n)}(t+s-u, w, y) d u \\
= & \int_{0}^{t} q_{x} e^{-q_{x}(u+s)} \sum_{w \neq x} Q_{x w} W^{(n)}(t-u, w, y) d u+\int_{0}^{s} q_{x} e^{-q_{x} u} \sum_{w \neq x} Q_{x w} W^{(n)}(t+s-u, w, y) d u
\end{aligned}
$$

tenemos que $0 \leq u \leq t$, entonces $s \leq u+s \leq t+s$ y haciendo $u=u+s$ obtenemos lo siguiente

$$
\begin{aligned}
& =\int_{s}^{t+s} q_{x} e^{-q_{x} u} \sum_{w \neq x} Q_{x w} W^{(n)}(t-(u-s), w, y) d u+\int_{0}^{s} q_{x} e^{-q_{x} u} \sum_{w \neq x} Q_{x w} W^{(n)}(t+s-u, w, y) d u \\
& =\int_{s}^{t+s} q_{x} e^{-q_{x} u} \sum_{w \neq x} Q_{x w} W^{(n)}(t+s-u, w, y) d u+\int_{0}^{s} q_{x} e^{-q_{x} u} \sum_{w \neq x} Q_{x w} W^{(n)}(t+s-u, w, y) d u \\
& =\int_{0}^{t+s} q_{x} e^{-q_{x} u} \sum_{w \neq x} Q_{x w} W^{(n)}(t+s-u, w, y) d u \\
& =W^{(n+1)}(t+s, x, y) .
\end{aligned}
$$

Ejemplo 16. (Proceso de Nacimiento y Muerte). Sea $S=\mathbb{N}$ y $q_{x}$ el parámetro de la distribución exponencial del tiempo de estancia en el estado $x$. Sea $Q$ la matriz estocástica de una cadena de Nacimiento y Muerte con

$$
r_{x}=p(x, y), \quad s_{x}=p(x, x-1), \quad p_{x}=p(x, x+1)
$$


entonces

$$
Q=\left(\begin{array}{ccccccc}
r_{0} & p_{0} & 0 & 0 & 0 & 0 & \ldots \\
s_{1} & r_{1} & p_{1} & 0 & 0 & 0 & \ldots \\
0 & s_{2} & r_{2} & p_{2} & 0 & 0 & \ldots \\
\vdots & \vdots & \vdots & \vdots & \vdots & \vdots & \ddots
\end{array}\right)=\left(\begin{array}{ccccccc}
0 & 1 & 0 & 0 & 0 & 0 & \ldots \\
s_{1} & 0 & p_{1} & 0 & 0 & 0 & \ldots \\
0 & s_{2} & 0 & p_{2} & 0 & 0 & \ldots \\
\vdots & \vdots & \vdots & \vdots & \vdots & \vdots & \ddots
\end{array}\right)
$$

En donde haciendo $r_{x}=0$ con $x \in \mathbb{N}$ estamos asegurando que $Q_{x x}=0$. Con la ecuación 3.5 construimos el generador infinitesimal de un proceso de Nacimiento y Muerte. Así

$$
L=\left(\begin{array}{ccccc}
-q_{0} & 0 & 0 & 0 & \ldots \\
0 & -q_{1} & 0 & 0 & \ldots \\
0 & 0 & -q_{2} & 0 & \ldots \\
\vdots & \vdots & \vdots & \vdots & \ddots
\end{array}\right)\left[\left(\begin{array}{ccccc}
0 & 1 & 0 & 0 & \ldots \\
s_{1} & 0 & p_{1} & 0 & \ldots \\
0 & s_{2} & 0 & p_{2} & \ldots \\
\vdots & \vdots & \vdots & \vdots & \ddots
\end{array}\right)-\left(\begin{array}{ccccc}
1 & 0 & 0 & 0 & \ldots \\
0 & 1 & 0 & 0 & \ldots \\
0 & 0 & 1 & 0 & \ldots \\
\vdots & \vdots & \vdots & \vdots & \ddots
\end{array}\right)\right]
$$

Resolviendo la multiplicación de matrices tenemos que el generador infinitesimal de un proceso de Nacimiento y muerte es,

$$
L=\left(\begin{array}{ccccc}
-q_{0} & q_{o} & 0 & 0 & \cdots \\
s_{1} q_{1} & -q_{1} & p_{1} q_{1} & 0 & \cdots \\
0 & s_{2} q_{2} & -q_{2} & p_{2} q_{2} & \cdots \\
\vdots & \vdots & \vdots & \vdots & \ddots
\end{array}\right)
$$

Sean $\lambda_{x}=q_{x} p_{x} y \mu_{x}=q_{x} s_{x} \cdot \lambda_{x}$ y $\mu_{x}$ son llamadas las intensidades de Nacimiento y Muerte respectivamente. Donde el generador respecto a las intensidades es

$$
L=\left(\begin{array}{ccccc}
-\left(\lambda_{0}+\mu_{0}\right) & \lambda_{0}+\mu_{0} & 0 & 0 & \cdots \\
\mu_{1} & -\left(\lambda_{1}+\mu_{1}\right) & \lambda_{1} & 0 & \cdots \\
0 & \mu_{2} & -\left(\lambda_{2}+\mu_{2}\right) & \lambda_{2} & \cdots \\
\vdots & \vdots & \vdots & \vdots & \ddots
\end{array}\right)
$$

\subsubsection{Proceso de Poisson}

Uno de los ejemplos más importantes de proceso de Markov a tiempo continuo es el proceso de Poisson el cual es un proceso de Nacimiento y Muerte donde la intensidad de muerte $\mu_{x}=0$ y $\lambda_{x}=\lambda>0$. A este tipo de procesos se les llama procesos puros de Nacimiento. Así que revisaremos características de este proceso.

Definición 30. Sea $T=\mathbb{R}_{+}$. Un proceso de Poisson con parámetro $\lambda>0$ es un proceso a tiempo continuo $\left\{X_{t}\right\}_{t \in T}$ con espacio de estados $S=\mathbb{N}$ y con trayectorias no decrecientes $y$ tal que 
i) $X_{0}=0$.

ii) Tiene incrementos independientes, es decir, con $t_{1}<t_{2}<t_{3}<\ldots$ las variables aleatorias $X_{t_{1}}, X_{t_{2}}-X_{t_{1}}, X_{t_{3}}-X_{t_{2}}, \ldots$ son independientes.

iii) $X_{t+s}-X_{s}$ tienen distribución de Poisson con parámetro $\lambda t$ para todo $s \geq 0, t>0$.

Una forma de construir un proceso de Poisson la mostramos siguiendo a [14]. Sea $T_{1}, T_{2}, \ldots$ una sucesión de variables aleatorias independientes cada una con distribución exponencial con parámetros $(\lambda)$. El proceso $\left\{X_{t}\right\}_{t \in T}$ será de Poisson con parámetro $\lambda$ donde las variables $X_{t}$ las definimos como $X_{t}=\operatorname{máx}\left\{n \geq 1: T_{1}+\ldots+T_{n} \leq t\right\}$ con máx $\emptyset=0$. Por lo tanto $W_{n}=T_{1}+\ldots+T_{n}$ será el tiempo real en el que se observa la ocurrencia del $n$-ésimo evento. Se tiene que,

Proposición 4. Para todo $t>0$ y $x, y \in \mathbb{N}$ tenemos que

$$
P\left(X_{t+s}=y \mid X_{s}=x\right)=e^{-\lambda t} \frac{(\lambda t)^{y-x}}{(y-x) !}
$$

Demostración. Sean $x, y \in S y x \leq y$,

$$
\begin{aligned}
P\left[X_{t+s}=y \mid X_{s}=x\right] & =P\left[X_{t+s}-X_{s}+X_{s}=y \mid X_{s}=x\right] \\
& =P\left[X_{t+s}-X_{s}=y-x \mid X_{s}=x\right] \\
& =P\left[X_{t+s}-X_{s}=y-x\right] \\
& =e^{-\lambda t} \frac{(\lambda t)^{y-x}}{(y-x) !}
\end{aligned}
$$

\subsubsection{Proceso de Poisson Compuesto}

Ahora sigamos a [14] y consideremos una Cadena de Markov $\left\{Y_{n}\right\}_{n=0}^{\infty}$ en $S$ donde $P\left(Y_{0} \in\right.$ $A)=\tilde{\pi}(A)$ y $P\left(Y_{n+1} \in A \mid Y_{0}, \ldots, Y_{n}\right)=Q\left(Y_{n}, A\right)$ con $A \in \mathfrak{B}(S)$ y $n \in \mathbb{N}$. Sean $\tau_{i}$ con $i \in \mathbb{Z}_{+}$ los tiempos cuando se realiza el salto hacia el estado $x_{i}$. Sea $\left\{X_{t}\right\}$ un proceso de Markov en $S$ con distribución de probabilidad $\pi$ definido como en la Definición 25 y generador

$$
L f(x)=q_{x} \int(f(y)-f(x)) Q(x, d y)
$$


Si $\left\{Y_{n}^{\prime}\right\}_{n=0}^{\infty}$ es Cadena de Markov en S con distribución $\pi$ y función de transición $Q^{\prime}(x, A)$ y sea $G$ un proceso de Poisson independiente con parámetro $q$. Definimos

$$
X_{t}^{\prime}=Y^{\prime}\left(G_{t}\right), \quad t \geq 0
$$

como un proceso de Poisson compuesto. La conclusión es que tanto $X$ como $X^{\prime}$ tienen las mismas distribuciones finito dimensionales. En otras palabras, son equivalentes en el sentido de la definición 8 sin que necesariamente uno sea modificación del otro. Es decir, un proceso de Saltos de Markov cuyas intensidades de saltos son una sucesión acotada se puede ver como un proceso de Poisson compuesto. Ver [3].

Supongamos que $q=\sup _{x \in S} q_{x}$ y $q<\infty$, definiendo

$$
Q^{\prime}(x, A)=\left(1-\frac{q_{x}}{q}\right) \delta_{x}(A)+\frac{q_{x}}{q} Q(x, A) .
$$

Entonces

$$
\begin{aligned}
L^{\prime} f(x) & =q \int(f(y)-f(x)) Q^{\prime}(x, d y) \\
& =q \int(f(y)-f(x))\left[\left(1-\frac{q_{x}}{q}\right) \delta_{x}(A)+\frac{q_{x}}{q} Q(x, A)\right] \\
& =q \frac{q_{x}}{q} \int(f(y)-f(x)) Q(x, d y) \\
& =q_{x} \int(f(y)-f(x)) Q(x, d y) \\
& =L f(x)
\end{aligned}
$$

Teorema 25. П es distribución invariante del proceso de Poisson compuesto si y sólo si П es distribución invariante de su cadena encajada.

Demostración. Sea L el generador infinitesimal del proceso compuesto. La ecuación (3.8) muestra que $L$ se puede tener como $L=q\left(Q^{\prime}-I\right)$, multiplicando por $\Pi$ tenemos que

$$
\Pi L=q \Pi\left(Q^{\prime}-I\right)=q\left(\Pi Q^{\prime}-\Pi\right) .
$$

Por lo tanto $\Pi L=0$ si y sólo si $\Pi Q^{\prime}=\Pi$. 


\subsection{Balance Detallado}

Sea $S$ espacio de estados a lo más numerable. Para un proceso de Markov $\left\{X_{t}\right\}_{t \in T}$ en tiempo continuo, la condición de balance detallado la definimos con el generador infinitesimal.

Definición 31. Decimos que $\mu$ está en balance detallado respecto al generador infinitesimal $L$ si

$$
\mu(x) L(x, y)=\mu(y) L(y, x) \quad \text { con } x, y \in S
$$

Ejemplo 17. (Proceso con dos estados). Sea $T=\mathbb{R}_{+}$. Consideremos el proceso de Markov $\left\{X_{t}\right\}_{t \in T}$ con dos posibles estados con generador infinitesimal

$$
\boldsymbol{L}=\left(\begin{array}{ll}
L(0,0) & L(0,1) \\
L(1,0) & L(1,1)
\end{array}\right)
$$

donde $L(x, y) \geq 0$ con $x, y \in S y \sum_{y} L(x, y)=1$. En este caso la única distribución invariante del proceso es

$$
\gamma=\left(\frac{L(1,0)}{L(1,0)-L(0,1)}, \frac{L(0,1)}{L(1,0)-L(0,1)}\right)
$$

y está en balance detallado.

Ejemplo 18. (Proceso con tres estados). Sea $T=\mathbb{R}_{+} y\left\{X_{t}\right\}_{t \in T}$ un proceso con tres posibles estados donde $Q^{\prime}$ es la matriz de transición modificada por la ecuación 3.8 la cual satisface la condición de reversibilidad de Kolmogorov

$$
Q^{\prime}=\left(\begin{array}{lll}
Q_{00}^{\prime} & Q_{01}^{\prime} & Q_{02}^{\prime} \\
Q_{10}^{\prime} & Q_{11}^{\prime} & Q_{12}^{\prime} \\
Q_{20}^{\prime} & Q_{21}^{\prime} & Q_{22}^{\prime}
\end{array}\right)
$$

y $q_{x}$ con $x \in S$ es el parámetro de la distribución exponencial de los tiempos de permanencia. Por tanto, el generador infinitesimal del proceso de Markov $\left\{X_{t}\right\}_{t \in T}$ es

$$
L=\left(\begin{array}{ccc}
-q_{0} & q_{0} Q_{01}^{\prime} & q_{0} Q_{02}^{\prime} \\
q_{1} Q_{10}^{\prime} & -q_{1} & q_{1} Q_{12}^{\prime} \\
q_{2} Q_{20}^{\prime} & q_{2} Q_{21}^{\prime} & -q_{2}
\end{array}\right)
$$

Sea $\mu=(\mu(0), \mu(1), \mu(2))$ distribución de probabilidad. Resolviendo las ecuaciones 3.10 para tener balance detallado obtenemos que

$$
\begin{aligned}
& \mu(0)=\frac{q_{1} Q_{10}^{\prime} q_{0} Q_{02}^{\prime}}{q_{1} Q_{10}^{\prime} q_{2} Q_{20}^{\prime}+q_{0} Q_{01}^{\prime} q_{0} Q_{02}^{\prime}+q_{0} Q_{02}^{\prime} q_{1} Q_{10}^{\prime}} \\
& \mu(1)=\frac{q_{0} Q_{01}^{\prime} q_{0} Q_{02}^{\prime}}{q_{1} Q_{10}^{\prime} q_{2} Q_{20}^{\prime}+q_{0} Q_{01}^{\prime} q_{0} Q_{02}^{\prime}+q_{0} Q_{02}^{\prime} q_{1} Q_{10}^{\prime}}
\end{aligned}
$$




$$
\mu(2)=\frac{q_{0} Q_{02}^{\prime} q_{1} Q_{10}^{\prime}}{q_{1} Q_{10}^{\prime} q_{2} Q_{20}^{\prime}+q_{0} Q_{01}^{\prime} q_{0} Q_{02}^{\prime}+q_{0} Q_{02}^{\prime} q_{1} Q_{10}^{\prime}}
$$

con $q_{1} Q_{10}^{\prime} \neq 0$ y $q_{2} Q_{20}^{\prime} \neq 0$. Además si $q_{x} Q_{x y}^{\prime}>0$ entonces $q_{y} Q_{y x}^{\prime}>0$ con $x, y \in S$.

Ejemplo 19. Sea $Q$ la matriz de transición del ejemplo 12 la cual la podemos sumergir el un proceso de Poisson para obtener el generador infinitesimal del proceso de Markov en tiempo continuo, el cual por 3.7 siendo $q=\sup _{x} q_{x}$, será

$$
L=\left(\begin{array}{ccc}
-q & q & 0 \\
0 & -q & q \\
q & 0 & -q
\end{array}\right)
$$

el cual nuevamente no está en balance detallado, es decir, no se satisfacen las ecuaciones 3.10. Sin embargo su única distribución invariante existe y es $\mu=\left(\frac{1}{3}, \frac{1}{3}, \frac{1}{3}\right)$.

\subsubsection{Proceso con espacio de estados finito}

Dado $\left\{X_{t}\right\}_{t \in T}$ un proceso de Markov en tiempo continuo con espacio de estados finito nos proponemos encontrar su distribución invariante y ponerla en términos de la distribución invariante de la cadena encajada $Q$ donde $Q_{x}=0$ para todo $x \in S$. Siguiendo a [14] consideremos el proceso de Markov sumergido en un proceso de Poisson compuesto tomando $q=\sup _{x} q_{x}$ con $x \in S$ con $q>0$. Sea $Q_{x y}^{\prime}$ como en 3.8 y sea $\pi$ su distribución invariante, entonces

Teorema 26. Supongamos que $Q$ tiene una única distribución invariante $\pi$ entonces $Q^{\prime}$ tiene una única distribución invariante $\pi^{\prime}$ dada por

$$
\pi^{\prime}(y)=A q \frac{\pi(y)}{q_{y}}=\frac{\pi(y) / q_{y}}{\sum_{y} \pi(y) / q_{y}}
$$

$\operatorname{con} A q=\left(\sum_{y} \frac{\pi(y)}{q_{y}}\right)^{-1}$.

Demostración.

$$
\begin{aligned}
\pi^{\prime}(y) & =\sum_{x \in S} \pi^{\prime}(x) Q_{x y}^{\prime} \\
& =\sum_{x} \pi^{\prime}(x)\left[\left(1-\frac{q_{x}}{q}\right) \delta_{x y}+\frac{q_{x}}{q} Q_{x y}\right] \\
& =\sum_{x} \pi^{\prime}(x) \delta_{x y}-\sum_{x} \pi^{\prime}(x) \frac{q_{x}}{q} \delta_{x y}+\sum_{x} \pi^{\prime}(x) \frac{q_{x}}{q} Q_{x y} \\
& =\pi^{\prime}(y)-\pi^{\prime}(y) \frac{q_{y}}{q}+\sum_{x} \pi^{\prime}(x) \frac{q_{x}}{q} Q_{x y}
\end{aligned}
$$


y obtenemos que

$$
\pi^{\prime}(y) \frac{q_{y}}{q}=\sum_{x} \pi^{\prime}(x) \frac{q_{x}}{q} Q_{x y}
$$

Sea $A=\sum_{y} \pi^{\prime}(y) \frac{q_{y}}{q}$ y dividiendo la ecuación anterior entre $A$

$$
\frac{\pi^{\prime}(y) \frac{q_{y}}{q}}{A}=\frac{\sum_{x} \pi^{\prime}(x) \frac{q_{x}}{q} Q_{x y}}{A}
$$

Entonces la única distribución invariante de $Q$ es

$$
\pi(y)=\frac{\pi^{\prime}(y) \frac{q_{y}}{q}}{A}
$$

por tanto $A \pi(y) q=\pi^{\prime}(y) q_{y}$ y así

$$
\sum_{y} \pi^{\prime}(y)=\sum_{y} \frac{A q}{q_{y}} \pi(y)
$$

Por otro lado tenemos que

$$
1=\sum_{y} \pi^{\prime}(y)=\sum_{y} \frac{A q}{q_{y}} \pi(y)=A q \sum_{y} \frac{\pi(y)}{q_{y}}
$$

y $A q=\left(\sum_{y} \frac{\pi(y)}{q_{y}}\right)^{-1}$. Por lo tanto

$$
\pi^{\prime}(y)=A q \frac{\pi(y)}{q_{y}}=\frac{\pi(y) / q_{y}}{\sum_{y} \pi(y) / q_{y}}
$$

Teorema 27. Dado $\left\{X_{t}\right\}_{t \in T}$ proceso de Markov con espacio de estados finito, si $Q^{\prime}$ tiene única distribución invariante $\pi^{\prime}$ entonces $\left\{X_{t}\right\}_{t \in T}$ tiene como distribución $\pi^{\prime}$.

Demostración. Puesto que $\pi^{\prime}(y)$ es la única distribución invariante entonces $Q_{x y}^{\prime}$ es irreducible y recurrente. Entonces ésta distribución invariante $\pi^{\prime}$ es distribución invariante del generador infinitesimal L, es decir, del proceso original.

Entonces $L$ es el generador infinitesimal del proceso de Markov $\left\{X_{t}\right\}_{t \in T}$ con espacio de estados finito y sea $D(H)$ el determinante de $L$ con $H \subset S$ subconjunto de índices. Denotemos por $\left\{\widetilde{X}_{n}\right\}_{n \geq 0}$ a la cadena encajada con $Q_{i j}$ matriz de transición y sea $\mathbf{D}=I-Q$ así $\widetilde{D}(H)$ es el determinante de $\mathbf{D}$ con $H$ conjunto de índices. 
Teorema 28. La única distribución de probabilidad invariante $\pi^{\prime}=\left\{\pi_{x}^{\prime}\right\}_{x \in S}$ del proceso de Markov es

$$
\pi_{x}^{\prime}=\frac{\pi_{x} / q_{x}}{\sum_{y \in S} \pi_{y} / q_{y}}=\frac{D\left(\{x\}^{c}\right)}{\sum_{y \in S} D\left(\{y\}^{c}\right)}, \quad \forall x \in S .
$$

Demostración. Para la primera igualdad de la ecuación (2.10) ya fue probada anteriormente y para la segunda igualdad de ésta ecuación tenemos lo siguiente

$$
\begin{aligned}
\pi_{x}^{\prime} & =\frac{\pi_{x} / q_{x}}{\sum_{y \in S} \pi_{y} / q_{y}} \\
& =\frac{\widetilde{D}\left(\{x\}^{c}\right) / q_{x}}{\sum_{y} \widetilde{D}\left(\{y\}^{c}\right) / q_{y}}
\end{aligned}
$$

pero por lo anterior tenemos que $-D\left(\{x\}^{c}\right)=\prod_{z} \frac{1}{q_{z}} \widetilde{D}\left(\{x\}^{c}\right) / q_{x}$ por tanto

$$
\begin{aligned}
\pi_{x}^{\prime} & =\frac{\widetilde{D}\left(\{x\}^{c}\right) / q_{x}}{\sum_{y} \widetilde{D}\left(\{y\}^{c}\right) / q_{y}} \\
& =\frac{\frac{-1}{\prod_{z} \frac{1}{q_{z}}} D\left(\{x\}^{c}\right)}{\frac{-1}{\prod_{z} \frac{-1}{q_{z}}} \sum_{y} D\left(\{y\}^{c}\right)} \\
& =\frac{D\left(\{x\}^{c}\right)}{\sum_{y} D\left(\{y\}^{c}\right)}
\end{aligned}
$$

Teorema 29. Sea $S$ espacio de estados finito y supongamos que $\sup \left\{q_{x}\right\}_{x=0}^{\infty}<\infty$. El proceso en tiempo continuo está en balance detallado si y sólo si la cadena encajada está en balance detallado.

Demostración. Sea $\pi^{\prime}$ la distribución invariante del proceso en tiempo continuo y $\pi$ la distribución invariante de la cadena encajada. Entonces por el Teorema 25

$$
\pi_{x}^{\prime}=\frac{\pi_{x} / q_{x}}{\sum_{y} \pi_{y} / q_{y}}
$$

Supongamos que $\pi^{\prime}$ está en balance detallado, entonces para $L$ su generador infinitesimal se tiene que $\pi_{x}^{\prime} L(x, y)=\pi_{y}^{\prime} L(y, x)$ con $x \neq y \in S$, pero $L(x, y)=q_{x} Q_{x y}$, así

$$
\begin{aligned}
\pi_{x}^{\prime} L(x, y) & =\pi_{x}^{\prime} q_{x} Q_{x y} \\
& =\frac{\pi_{x} Q_{x y}}{\sum_{y} \pi_{y} / q_{y}}
\end{aligned}
$$


por otro lado

$$
\begin{aligned}
\pi_{y}^{\prime} L(y, x) & =\frac{\pi_{y} Q_{y x}}{\sum_{z} \pi_{z} / q_{z}} \\
& =\pi_{y}^{\prime} q_{y} Q_{y x}
\end{aligned}
$$

$y$ entonces $\pi_{x} Q_{x y}=\pi_{y} Q_{y x}$.

Ejemplo 20. (Modelo de Aprendizaje y Olvido). Recordemos que el generador infinitesimal del proceso es

$$
\boldsymbol{L}=\left(\begin{array}{cccccc}
v_{A_{1} A_{1}} & v_{A_{1} A_{2}} & \mu_{A_{1} f_{1}} & \mu_{A_{1} f_{2}} & \ldots & \mu_{A_{1} f_{r}} \\
v_{A_{2} A_{1}} & v_{A_{2} A_{2}} & \mu_{A_{2} f_{1}} & \mu_{A_{2} f_{2}} & \ldots & \mu_{A_{2} f_{r}} \\
0 & 0 & 0 & 0 & \ldots & 0 \\
0 & 0 & 0 & 0 & \ldots & 0 \\
\vdots & \vdots & \vdots & \vdots & \ddots & \vdots \\
0 & 0 & 0 & 0 & \ldots & 0
\end{array}\right)
$$

Como podemos observar en el generador infinitesimal, si $\gamma$ es distribución invariante

$$
\gamma_{1} \cdot 0=\gamma_{1} L(1,2) \neq \gamma_{2} L(2,1)=\gamma_{2} \mu_{A_{1} f_{1}}
$$

es decir, $\gamma$ no está en balance detallado, por tanto este proceso no es reversible, es decir, es un sistema fuera de equilibrio. Se puede pensar en éste modelo el no equilibrio como el aprendizaje el cual no se puede corregir (revertir) para que se tenga una mejora en éste como lo es al aprender a manejar, se aprende de cierta manera en la cual es difícil de corregir las fallas al conducir.

\subsubsection{Proceso con espacio de estados infinitos numerable.}

En esta sección consideraremos algunos ejemplos de proceso de Markov en tiempo continuo, es decir, $T=\mathbb{R}$ y $S$ espacio de estados infinito.

Ejemplo 21. Consideremos nuevamente un proceso de Nacimiento y Muerte como en (2.3.1) con $\lambda_{x}$ intensidad de nacimiento y $\mu_{x}$ intensidad de muerte. Para saber si este proceso está en 
balance detallado, consideremos una medida de probabilidad $\varrho(x)>0$ con $\sum_{x} \varrho(x)=1$. Recordemos que el generador infinitesimal de este proceso es

$$
L=\left(\begin{array}{ccccc}
-q_{0} & q_{0} & 0 & 0 & \ldots \\
s_{1} q_{1} & -q_{1} & p_{1} q_{1} & 0 & \ldots \\
0 & s_{2} q_{2} & -q_{2} & p_{2} q_{2} & \ldots \\
\vdots & \vdots & \vdots & \vdots & \ddots
\end{array}\right)
$$

usando las ecuaciones de balance detallado obtenemos que la distribución invariante del proceso es

$$
\varrho(x)=\prod_{y=0}^{x} \frac{\lambda_{y}}{\mu_{y+1}} \varrho(0) \quad \text { con } \quad \varrho(0)=\left(\sum_{x=0}^{\infty} \prod_{y=0}^{x} \frac{\lambda_{y}}{\mu_{y+1}}\right)^{-1}
$$

pero ésta no siempre existe, sólo si la serie es convergente. Por lo tanto un proceso de Nacimiento y Muerte tiene distribución invariante en balance detallado si la serie

$$
\sum_{x=0}^{\infty} \prod_{y=0}^{x} \frac{\lambda_{y}}{\mu_{y+1}}
$$

es convergente, además @ será única. Si queremos calcular la distribución invariante de este proceso nos da como resultado que ésta existirá si y sólo si la serie 3.12 converge por lo tanto tenemos el siguiente resultado.

Teorema 30. Sea $\left\{X_{t}\right\}_{t \in T}$ un proceso de Nacimieno y Muerte con intensidades $\lambda_{x}$ y $\mu_{x}$. El proceso está en balance detallado si y sólo si el proceso tiene distribución invariante.

Demostración. Sabemos que si el proceso está en balance detallado, entonces tiene distribución invariante. Ahora, si el proceso tiene distribución invariante $\pi$ se debe cumplir que $\sum_{i=0}^{\infty} \pi(i) L(i, j)=0$ lo cual se cumple si y sólo si la serie 3.12 converge lo que implica que ésta esté en balance detallado.

Ejemplo 22. Consideremos nuevamente un proceso de Nacimiento y Muerte con intensidades 
tales que $\lambda_{x}=\lambda(x+1)^{2}$ y $\mu_{x}=\mu x^{2}+x=x(x+1)$, por lo tanto de la serie 3.12 tenemos que

$$
\begin{aligned}
\sum_{x=0}^{\infty} \prod_{y=0}^{x} \frac{\lambda(x+1)^{2}}{\mu[(x+1)(x+2)]} & =\sum_{x=0}^{\infty} \prod_{y=0}^{x} \frac{\lambda(x+1)}{\mu(x+2)} \\
& =\sum_{x=0}^{\infty}\left(\frac{\lambda}{\mu}\right)^{x} \prod_{y=0}^{x} \frac{x+1}{x+2} \\
& =\sum_{x=0}^{\infty}\left(\frac{\lambda}{\mu}\right)^{x}\left(\frac{1}{x+2}\right) \\
& <\sum_{x=0}^{\infty}\left(\frac{\lambda}{\mu}\right)^{x}
\end{aligned}
$$

donde la serie 3.12 converge si y sólo si $\lambda<\mu$, es decir, la intensidad de nacimiento es menor que la intensidad de muerte y por lo tanto en este caso, se tendría balance detallado.

Ejemplo 23. Consideremos la cadena de Markov definida en el Ejemplo 13 donde se tiene una línea de espera con un servidor y espacio de estados infinito. Si a la línea de espera la sumergimos en un proceso de Poisson compuesto con $q_{x}$ y $q=\sup q_{x}<\infty$ como en 3.7 obtenemos que el generador infinitesimal del proceso en tiempo continuo es $L=\lambda(Q-I)$, es decir,

$$
L=\left(\begin{array}{ccccc}
\lambda(g(0)-1) & \lambda g(1) & \lambda g(2) & \lambda g(3) & \ldots \\
\lambda g(0) & \lambda(g(1)-1) & \lambda g(2) & \lambda g(3) & \ldots \\
0 & \lambda g(0) & \lambda(g(1)-1) & \lambda g(2) & \ldots \\
\vdots & \vdots & \vdots & \vdots & \ddots
\end{array}\right)
$$

Con distribución invariante la misma que Q, calculada en el Ejemplo 13, la cual nuevamente no está en balance detallado por el Teorema 24. 


\section{Capítulo 4}

\section{Equilibrio en el sentido de Gibbs}

En este capítulo revisaremos el formalismo termodinámico de los sistemas clásicos lattice, ya que el objetivo es estudiar algunas nociones de equilibrio. Como condición de equilibrio, en el capítulo anterior, teníamos el balance detallado, aquí tendremos otra noción de Equlibrio.

El equilibrio termodinámico se caracteriza por tener un valor mínimo en sus potenciales termodinámicos, es decir, variables de estado que tienen dimensiones de energía, tales como la energía libre de Gibbs, son sistemas caracterizados por tener la presión y temperatura constantes. Un estado de equilibrio en un sistema lattice finito es aquel que minimiza la energía libre de Gibbs, lo cual se llama equilibrio termodinámico.

Los sistemas físicos para los cuales la mecánica estadística en equilibrio se aplica son idealizados infinitos, es decir, para ocupar $\mathbb{R}^{N}$ donde $N$ representa las $N$ partículas del sistema. Esta utopía es necesaria puesto que sólo sistemas infinitos exhiben fuertemente la transformación del sistema de una fase a otra. En este trabajo nos concentramos en los sistemas clásicos lattice donde $\mathbb{R}^{N}$ es reeemplazada por $\mathbb{Z}^{N}$.

Los sistemas clásicos lattice son modelos matemáticos de cristales que consisten de muchos subsistemas, cada subsistema puede tener una infinidad de estados. Nos basamos en [8], [9]. Los sistemas lattice son los sistemas clásicos más simples, y se considera la lattice en $\mathbb{Z}^{N}$. Para tales sistemas el espacio de configuraciones es un subconjunto $\Omega$ de $\prod_{x \in \mathbb{Z}^{N}} \Omega_{x}$, donde $\Omega_{x}$ es el conjunto de posibles números ocupados en el sitio $x$ del lattice ó bien un conjunto de símbolos. 
Sea $(S, \mathfrak{S})$ un espacio medible, y $T$ el conjunto definido por $T=\mathbb{Z}^{N}$ con $\mathfrak{S}$ una $\sigma$-álgebra generada sobre $S$ y $(\Omega, \mathfrak{F}, P)$ espacio de probabilidad con $\Omega=\Omega_{0}^{T}$ y $\mathfrak{F}=\mathcal{C}(\Omega)$, es decir, la $\sigma$-álgebra cilíndrica.

\subsection{Sistemas Lattice Finitos}

Definición 32. Sea $\Lambda$ un subconjunto finito de T. El espacio de configuraciones $\Omega_{\Lambda}$ de un sistema lattice es el conjunto $\Omega_{\Lambda}=\times_{a \in \Lambda} \Omega_{a}$ donde $\Omega_{a}=\{0,1, \ldots, k\}$ con $k \in \mathbb{N}$, es decir, es el conjunto de posibles números ocupados en a.

Definición 33. Una configuración de un sistema lattice en $\Lambda$ es un punto en $\Omega_{\Lambda}$ y un estado de un sistema lattice en $\Lambda$ es una medida de probabilidad sobre $\Omega_{\Lambda}$.

Observemos que $\Lambda$ es un subconjunto acotado de $T$, por ser finito), y que $\Omega_{\Lambda}$ es un conjunto finito y cada estado en el espacio de configuraciones puede ser dado como una distribución de probabilidad discreta $P=\left\{P(\omega): \omega \in \Omega_{\Lambda}\right\}$.

Definición 34. Dado un estado $P$ del sistema, la entropía de $P$ está dada por

$$
S(P)=-\sum_{\omega \in \Omega_{\Lambda}} P(\omega) \log P(\omega)
$$

donde, como convención $t \log t=0$ sit $t=0$.

Una función $U: \Omega_{\Lambda} \longrightarrow \mathbb{R}$ es llamada función de energía. Esta función es la energía potencial la cual mide la capacidad que tiene el sistema para realizar un trabajo en función unicamente de su configuración o posición.

Definición 35. Dado un estado P y una función de energía U, la energía total del sistema en el estado $P$ es tomada como el valor esperado de la función de energía $U$ tal que

$$
E_{T}=\sum_{\omega \in \Omega_{\Lambda}} P(\omega) U(\omega)
$$

Definición 36. (Ensamble Canónico de Gibbs). Dada una función de energía U, el ensamble canónico de Gibbs es el estado del sistema dado por

$$
P_{G}(\omega)=\frac{e^{-\beta U(\omega)}}{\sum_{\omega \in \Omega_{\Lambda}} e^{-\beta U(\omega)}}
$$


donde $\beta$ es una constante. El denominador $Z=\sum_{\omega \in \Omega_{\Lambda}} e^{-\beta U(\omega)}$ es llamada la función de partición.

Esta definición es la dada por [9], cabe mencionar que en la definición dada de ensamble de Gibbs en [8] $\beta=1$. Si hacemos $\beta=(k T)^{-1}$ con $k$ la constante de Boltzman $\left(k=1.3806504 \times 10^{-23} \mathrm{~J} / \mathrm{K}\right)$.

Teorema 31. (Principio variacional). Sea $\Omega_{\Lambda}$ el espacio de configuraciones de un sistema lattice clásico en una región acotada $\Lambda$. Dada $U: \Omega \longrightarrow \mathbb{R}$ función de energía, la función $V$ definida sobre todos los estados $\sigma$ de $\Omega_{\Lambda}$ dada por

$$
V(\sigma)=S(\sigma)-\beta E_{T}
$$

alcanza su máximo en el ensamble canónico de Gibbs y el valor máximo de $V$ es $\log Z$.

Demostración. Ya que $\sigma$ es una distribución de probabilidad discreta, usaremos multiplicadores de Lagrange. Supongamos que $\sigma=\left(x_{1}, x_{2} \ldots x_{k}\right)$. Entonces deseamos encontrar el valor extremo de

$$
\begin{gathered}
\text { máx } V\left(x_{1}, \ldots, x_{k}\right)=-\sum_{i=1}^{k} x_{i} \log x_{i}-\beta \sum_{i=1}^{k} x_{i} U_{i} . \\
\text { sujeta } a \sum_{i=1}^{k} x_{i}=1 .
\end{gathered}
$$

en la expresión de $V, U_{i}$ denota la energía que se debe a la $i$-ésima configuración $\omega_{i}$. Para calcular el máximo de $V$, debemos mostrar que

$$
\begin{gathered}
\frac{\partial}{\partial x_{i}}\left(V+\lambda\left(\sum_{i=1}^{k} x_{i}-1\right)\right)=0, \text { o bien } \\
\frac{\partial}{\partial x_{i}}\left[-\sum_{i=1}^{k} x_{i} \log x_{i}-\beta \sum_{i=1}^{k} x_{i} U_{i}+\lambda \sum_{i=1}^{k} x_{i}-\lambda\right]=0
\end{gathered}
$$

derivando para cada $x_{i}$ tenemos que $-\log x_{i}-1-\beta U_{i}+\lambda=0$ entonces $-1-\beta U_{i}+\lambda=\log x_{i}$, así $x_{i}=e^{-\left(1+\beta U_{i}\right)+\lambda}$. Usando la restrición tenemos que

$$
1=\sum_{i=1}^{k} x_{i}=\sum_{j} e^{-\left(1+\beta U_{i}\right)} e^{\lambda}
$$

tomando logaritmo

$$
0=\lambda+\log \left(\sum_{j} e^{-\left(1+\beta U_{j}\right)}\right) \quad \text { asi } \quad \lambda=\log \left(\sum_{j} e^{-\left(1-\beta U_{j}\right)}\right)^{-1}
$$


Entonces el máximo es

$$
x_{i}=\frac{e^{-\beta U_{i}}}{\sum_{j=1}^{k} e^{-\beta U_{j}}}
$$

que es el ensamble de Gibbs. y el valor que toma la función es:

$$
\begin{aligned}
V\left(x_{i}\right) & =-\sum_{i=1}^{k}\left(\frac{e^{-\beta U_{i}}}{\sum_{j=1}^{k} e^{-\beta U_{j}}}\right)\left(-\beta U_{i}-\log \sum_{i=1}^{k} e^{-\beta U_{i}}\right)-\sum_{i=1}^{k} \frac{e^{-\beta U_{i}}}{\sum_{j=1}^{k} e^{-\beta U_{j}}} \beta U_{i} \\
& =\log \left(\sum_{j=1}^{k} e^{-\beta U_{j}}\right)=\log Z .
\end{aligned}
$$

que es el logaritmo de la función partición del ensamble canónico de Gibbs.

Notemos del Teorema de Principio Variacional que si $E_{T}$ es constante para cualquier estado entonces $U_{i}=$ constante, es decir, $\sum_{\omega \in \Omega_{\Lambda}} P(\omega) U(\omega)=E$ con $E$ constante, la ecuación 4.1 es simplemente $V(\sigma)=S(\sigma)-\beta$ con $\beta=$ constante.Por lo tanto el ensamble canónico de Gibbs $P_{G}$ maximiza la entropía $S(\sigma)$, es decir, el estado de Equlibrio de un sistema clásico lattice en una región finita con energía constante maximiza la entropía.

Observemos que si $G(\sigma)=-\frac{1}{\beta} V(\sigma)$ donde $V(\sigma)$ es como en la ecuación 4.1 a $G(\sigma)$ se le llama energía libre de Gibbs. De acuerdo con el principio variacional, el ensamble canónico de Gibbs minimiza la energía libre de Gibbs. Siendo $\beta$ la constante definida anteriormente, la energía libre de Gibbs es

$$
G(\sigma)=-\frac{1}{\beta} V(\sigma)=E_{T}(\sigma)-\frac{1}{\beta} S(\sigma)=E_{T}-k T S .
$$

\subsection{Sistemas Lattice Infinitos}

Sea $\Omega_{0}$ un conjunto finito de símbolos tal como $\Omega_{0}=\{0,1, \ldots, k\}$ con $k \in \mathbb{N}$ y $\Omega_{0}=\Omega_{x}$. Sea $\mathcal{F}=\{\Lambda \subset T: \Lambda$ finito $\}$. Observemos que $\mathcal{F}$ es un conjunto infinito numerable pues si $\mathcal{F}_{k}=\{A \subset T:|A|=k\}$ con $k \in \mathbb{N} \cup\{0\}$ y $|A|$ denota la cardinalidad de $A$, entonces $\mathcal{F}=\bigcup_{k=0}^{\infty} \mathcal{F}_{k}$. 
Definición 37. El espacio de configuraciones es el conjunto $\Omega=\Pi_{x \in T} \Omega_{x}$ donde $\Omega_{0}=\Omega_{x}$. Llamamos a $\Omega_{\Lambda}=\Pi_{x \in \Lambda} \Omega_{x}$ el espacio de configuraciones con base $\Lambda \subset T$

Recordemos que en sistemas lattice finitos tenemos que el espacio de configuraciones se define sólo para subconjunto finitos de $T$, en cambio para sistemas infinitos consideramos todo $T$.

Observación: Las configuraciones son puntos $\omega \in \Omega$, las cuales son funciones sobre $T$, tales que $\omega_{x} \in \Omega$ con $x \in T$ pues $\Omega$ es un producto cartesiano, es decir, $\omega: T \longrightarrow \Omega_{x}$.

Definición 38. Dados $\Lambda, \Lambda_{1} \in \mathcal{F}$ y $\Lambda_{1} \subset \Lambda_{2}$ definimos las proyecciones

$$
\begin{aligned}
\Pi_{\Lambda}: \Omega \rightarrow \Omega_{\Lambda}, \quad \text { con } & \Pi_{\Lambda}(\omega)=\left.\omega\right|_{\Lambda} \\
\Pi_{\Lambda_{1} \Lambda_{2}}: \Omega_{\Lambda_{2}} \rightarrow \Omega_{\Lambda_{1}}, \quad \text { con } & \Pi_{\Lambda_{1} \Lambda_{2}}(\omega)=\left.\omega\right|_{\Lambda_{2}}
\end{aligned}
$$

Esto es por que $\Omega_{\Lambda}$ no es un subconjunto de $\Omega$ sino que es un conjunto sobre el cual se puede proyectar $\Omega$, a través de $\Pi_{\Lambda}$, es decir, $\Omega_{\Lambda}$ es un factor de $\Omega$. Con más precisión, $\Omega$ se puede pensar como $\Omega_{\Lambda} \times \Omega_{\Lambda^{c}}$, con $\omega \in \Omega$ tal que $\omega=\left(\omega_{\Lambda} \cdot \Omega_{\Lambda^{c}}\right)$.

Sea $\mathcal{C}=\sigma\left(\Pi_{\Lambda}^{-1}(\omega)\right)$, es decir, la $\sigma$-álgebra cilíndrica sobre $\Omega_{\Lambda}$.

Definición 39. Un estado es cualquier medida de probabilidad sobre el espacio medible $(\Omega, \mathcal{C})$ y sea $\Xi$ la colección de todos los estados.

Definición 40. Para $a \in T$, la traslación por a es el mapeo $T_{a}: \Omega \longrightarrow \Omega$ tal que

$$
\left(T_{a} \omega\right)_{x}=\omega_{x+a}
$$

$\operatorname{con} x \in T y \omega \in \Omega$.

Definición 41. Sea $\bar{\Omega}=\bigcup\left\{\Omega_{\Lambda}: \Lambda \in \mathcal{F}\right\}$. Una interacción es una función $\Phi: \bar{\Omega} \rightarrow \mathbb{R}$ tal que

1. $\Phi(\emptyset)=0$

2. $\Phi\left(T_{a} \omega\right)=\Phi(\omega)$ con $a \in T$ y $\omega \in \Omega$. Esta propiedad es llamada invariancia por traslaciones. 
3. Para cada $x \in T$,

$$
|\Phi|_{x}=\sum_{\Lambda \ni x} \frac{1}{|\Lambda|} \sup _{\xi \in \Omega_{\Lambda}}|\Phi(\xi)|<+\infty
$$

Definición 42. Para una interacción $\Phi$ dada y $\Lambda \in \mathcal{F}$, la función de energía dada la interacción $\Phi$ es $U_{\Lambda}^{\Phi}: \Omega_{\Lambda} \rightarrow \mathbb{R}$ :

$$
U_{\Lambda}^{\Phi}(\omega)=\sum_{\Lambda^{\prime} \subset \Lambda} \Phi\left(\left.\omega\right|_{\Lambda^{\prime}}\right)
$$

Donde esta función es la energía potencial del sistema.

Teorema 32. Dada la interacción $\Phi$

$$
\begin{aligned}
& \text { 1. } U_{\Lambda}^{\Phi}(\xi)=\sum_{x \in \Lambda} \sum_{\Lambda^{\prime}: x \in \Lambda^{\prime} \subset \Lambda} \frac{1}{\left|\Lambda^{\prime}\right|} \Phi\left(\left.\xi\right|_{\Lambda^{\prime}}\right) \\
& \text { 2. }\left|U_{\Lambda}^{\Phi}\right| \leq \sum_{x \in \Lambda}|\Phi|_{x}
\end{aligned}
$$

Demostración. Para 1 tenemos que

$$
\begin{aligned}
\sum_{x \in \Lambda} \sum_{\Lambda^{\prime}: x \in \Lambda^{\prime} \subset \lambda} \frac{1}{\left|\Lambda^{\prime}\right|} \Phi\left(\left.\xi\right|_{\Lambda^{\prime}}\right) & =\sum_{\Lambda^{\prime} \subset \lambda} \sum_{x \in \Lambda^{\prime}} \frac{1}{\left|\Lambda^{\prime}\right|} \Phi\left(\left.\xi\right|_{\Lambda^{\prime}}\right)=\sum_{\Lambda^{\prime} \subset \Lambda} \frac{\left|\Lambda^{\prime}\right|}{\left|\Lambda^{\prime}\right|} \Phi\left(\left.\xi\right|_{\Lambda^{\prime}}\right) \\
& =\sum_{\Lambda^{\prime} \subset \Lambda} \Phi\left(\left.\xi\right|_{\Lambda^{\prime}}\right)=U_{\Lambda}^{\Phi}(\xi) .
\end{aligned}
$$

Para 2 se tiene que

$$
\begin{aligned}
\left|U_{\Lambda}^{\Phi}\right| & =\left|\sum_{\Lambda^{\prime} \subset \Lambda} \Phi\left(\left.\xi\right|_{\Lambda^{\prime}}\right)\right|=\left|\sum_{x \in \Lambda} \sum_{\Lambda^{\prime}: x \in \Lambda^{\prime} \subset \Lambda} \frac{1}{\left|\Lambda^{\prime}\right|} \Phi\left(\left.\xi\right|_{\Lambda^{\prime}}\right)\right| \\
& \leq \sum_{x \in \Lambda}\left|\sum_{\Lambda^{\prime}: x \in \Lambda^{\prime} \subset \Lambda} \frac{1}{\left|\Lambda^{\prime}\right|} \Phi\left(\left.\xi\right|_{\Lambda^{\prime}}\right)\right| \leq \sum_{x \in \Lambda} \sum_{\Lambda^{\prime}: x \in \Lambda^{\prime} \subset \Lambda} \frac{1}{\left|\Lambda^{\prime}\right|}\left|\Phi\left(\left.\xi\right|_{\Lambda^{\prime}}\right)\right| \\
& \leq \sum_{x \in \Lambda} \sum_{\Lambda^{\prime} \ni x} \frac{1}{\left|\Lambda^{\prime}\right|} \sup _{\xi \in \Omega_{\Lambda}}\left|\Phi\left(\left.\xi\right|_{\Lambda^{\prime}}\right)\right|=\sum_{x \in \Lambda}|\Phi|_{x}
\end{aligned}
$$

Siguiendo a [8] podemos dar una condición más fuerte para las interacciones que la dada en 3 de la Definición 41. Dada la interacción $\Phi$ definimos a $\|\Phi\|_{x}$ para todo $x$ por

$$
\|\Phi\|_{x}=\sum_{\Lambda \ni x} \sup _{\xi \in \Omega_{\Lambda}}|\Phi(\xi)| .
$$


donde $|\Phi|_{x} \leq\|\Phi\|_{x}$.

Definición 43. Dada la interacción $\Phi$ tal que $\|\Phi\|_{x}<\infty$ definimos a la interacción entre dos subconjuntos disjuntos $\Lambda_{1}, \Lambda_{2} \subset T$ con $\Lambda_{1} \in \mathcal{F}$ como la función $W_{\Lambda_{1} \Lambda_{2}}^{\phi}: \Omega \longrightarrow \mathbb{R}$ tal que

$$
W_{\Lambda_{1} \Lambda_{2}}^{\Phi}(\omega)=\sum_{\substack{\Lambda^{\prime} \subset \Lambda_{1} \cup \Lambda_{2} \\ \Lambda^{\prime} \cap \Lambda_{1} \neq \emptyset \\ \Lambda^{\prime} \cap \Lambda_{2} \neq \emptyset}} \Phi\left(\left.\omega\right|_{\Lambda^{\prime}}\right)
$$

Observación: $\quad$ Si $\left|\Lambda_{1}\right|=1$ entonces no existe $\Lambda^{\prime}$ tal que $\left|\Lambda^{\prime}\right|=1$ con $\Lambda^{\prime} \cap \Lambda_{1} \neq \emptyset$ y $\Lambda^{\prime} \cap \Lambda_{2} \neq \emptyset$ por lo tanto $W_{\Lambda_{1} \Lambda_{2}}^{\Phi}(\omega)=0$.

Proposición 5. Dada la interacción $\Phi$ si $\|\Phi\|_{x}<\infty$ para toda $x \in \Lambda$, entonces

$$
\left|W_{\Lambda_{1} \Lambda_{2}}^{\Phi}\right| \leq \sum_{x \in \Lambda}\|\Phi\|_{x}
$$

\section{Demostración.}

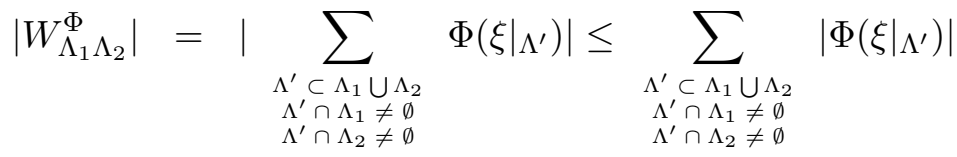

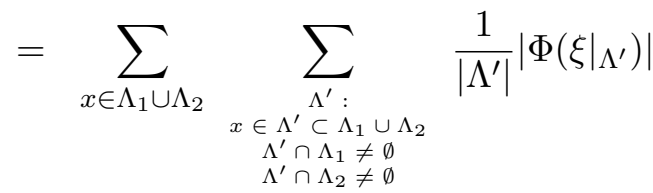

$$
\begin{aligned}
& \leq \sum_{x \in \Lambda} \sum_{\Lambda^{\prime}: x \in \Lambda^{\prime} \subset \Lambda} \frac{1}{\left|\Lambda^{\prime}\right|}\left|\Phi\left(\left.\xi\right|_{\Lambda^{\prime}}\right)\right| \\
& \leq \sum_{x \in \Lambda}|\Phi|_{x} \leq \sum_{x \in \Lambda}\|\Phi\|_{x}
\end{aligned}
$$

ya que, cuando $|\Lambda| \geq 1$

$$
\frac{1}{|\Lambda|} \sup _{\xi \in \Omega_{\Lambda}}|\Phi(\xi)| \leq \sup _{\xi \in \Omega_{\Lambda}}|\Phi(\xi)|
$$

Definición 44. (Ensamble de Gibbs). Dada la interacción $\phi$. Un ensamble de Gibbs asociado a $\phi$ es la distribución de probabilidad discreta $P_{\Lambda}: \Omega_{\Lambda} \longrightarrow \mathbb{R}$ tal que

$$
P_{\Lambda}\left(\omega_{0}\right)=e^{-U_{\Lambda}^{\phi}}\left(\omega_{0}\right) Z_{\Lambda}(\phi)^{-1}
$$

con $Z_{\Lambda}(\phi)^{-1}=\sum_{\omega \in \Omega_{\Lambda}} e^{-U_{\Lambda}^{\phi}(\omega)}$ llamada función de partición y $U_{\Lambda}^{\phi}$ es la función definida en 42. 
Observemos que el ensamble de Gibbs para sistemas infinitos depende de la interacción $\phi$, a diferencia del ensamble de Gibbs para sistemas finitos.

Consideremos $\left\{\Lambda_{n}\right\}$ una sucesión de subconjuntos de $\mathbb{Z}^{N}$ con $N \in \mathbb{N}$. Diremos que $\Lambda_{n}$ converge a $\mathbb{Z}^{N}$ si $\underline{\lim } \Lambda_{n}=\mathbb{Z}^{N}$ con $\underline{\lim } \Lambda_{n}=\sup _{k \geq 1} \operatorname{lnf}_{k \geq n} \Lambda_{k}$. Si $\Lambda_{n}$ converge a $\mathbb{Z}^{N}$, entonces cualquier subsucesión $\Lambda_{n}^{\prime}$ también converge a $\mathbb{Z}^{N}$ pues $\mathbb{Z}^{N}=\underline{\lim } \Lambda_{n} \subseteq \underline{\lim } \Lambda_{n}^{\prime} \subseteq \mathbb{Z}^{N}$, para toda $\Lambda_{n}^{\prime}$

Teorema 33. (Existencia del Límite Termodinámico). Sea $\left\{\Lambda_{n}\right\}$ una sucesión de subconjuntos finitos de $\mathbb{Z}^{N}$ la cual converge a $\mathbb{Z}^{N}$. Para cada $n$ sea $\mu_{\Lambda_{n}}$ una medida de probabilidad sobre $\Omega_{\Lambda_{n}}$. Entonces

i) Existe una subsucesión $\left\{\Lambda_{n}^{\prime}\right\}$ de $\left\{\Lambda_{n}\right\}_{n=1}^{\infty}$ tal que para cada conjunto finito $\Lambda \subset \mathbb{Z}^{N}$

$$
\lim _{n \rightarrow \infty} \mu_{\Lambda_{n}^{\prime}}\left\{p_{\Lambda \Lambda_{n}^{\prime}}^{-1}\{\omega\}\right\}
$$

existe para todo $\omega \in \Omega_{\Lambda}$.

ii) Sea $\rho_{\Lambda}\{\omega\}=\lim _{n \rightarrow \infty} \mu_{\Lambda_{n}^{\prime}}\left\{p_{\Lambda \Lambda_{n}^{\prime}}^{-1}\{\omega\}\right\}$. Entonces existe un único estado $\rho$ en $\Omega$ (es decir, una medida de probabilidad $\rho: \mathcal{C} \rightarrow[0,1]$ ) tal que para cada conjunto finito $\Lambda \subset \mathbb{Z}^{N}$

$$
\rho\left\{p_{\Lambda}^{-1}\{\omega\}\right\}=\rho_{\Lambda}\{\omega\}, \quad \omega \in \Omega_{\Lambda}
$$

donde $\rho\left\{p_{\Lambda}^{-1}\{\omega\}\right\}$ es la distribución finito dimensional de $\rho$ respecto a $\Lambda$

El estado $\rho$ se llama el límite termodinámico de la sucesión de estados $\mu_{\Lambda_{n}^{\prime}}$.

Demostración. Para probar i) seguiremos varios pasos.

Afirmación: Sea $\Lambda \in \mathcal{F}$ y sea $\left\{B_{n}\right\}_{n=1}^{\infty}$ una sucesión en $\mathcal{F}$ tal que $B_{n} \longrightarrow \mathbb{Z}^{N}$. Supongamos que $\Upsilon_{B_{n}}: 2^{\Omega_{B_{n}}} \longrightarrow[0,1]$ es una medida de probabilidad para todo $n$. Entonces existe $\left\{B_{n}^{\Lambda}\right\}_{n=1}^{\infty}$ subsucesión de $\left\{B_{n}\right\}_{n=1}^{\infty}$ tal que

$$
\lim _{n \rightarrow \infty} \Upsilon_{B_{n}^{\Lambda}}\left(p_{\Lambda B_{n}^{\Lambda}}^{-1}\{\omega\}\right)
$$

existe para todo $\omega$ en $\Lambda$.

Esto es porque si $S=\Omega_{\Lambda}, f_{n}: \Omega_{\Lambda} \longrightarrow[0,1] y$

$$
f_{n}(\omega)=\Upsilon_{B_{n}}\left(p_{\Lambda B_{n}}^{-1}\{\omega\}\right)
$$


como $\Omega_{\Lambda}$ es conjunto finito entonces por la técnica diagonal de Cantor existe una subsucesión $\left\{f_{n}^{\prime}\right\}_{n=1}^{\infty}$ de $\left\{f_{n}\right\}_{n=1}^{\infty}$ que converge puntualmente. Sea $B_{n}^{\prime}=B_{n}^{\Lambda}$, entonces

$$
\lim _{n \rightarrow \infty} \Upsilon_{B_{n}^{\Lambda}}\left(p_{\Lambda B_{n}^{\Lambda}}^{-1}\{\omega\}\right) \quad \text { existe, }
$$

ya que $\Upsilon_{B_{n}^{\Lambda}}\left(p_{\Lambda B_{n}^{\Lambda}}^{-1}\{\omega\}\right)=f_{n}^{\prime}(\omega)$.

Ahora sea $F=\left\{A_{1}, A_{2}, A_{3} \ldots\right\}$ una numeración del conjunto $\mathcal{F}$, es decir, numeración de todos los subconjuntos finitos de $\mathbb{Z}^{N}$.

Paso 1. Sea $\Lambda \in F$. Sabemos que $\left\{\Lambda_{n}\right\}_{n=1}^{\infty}$ es una sucesión en F tal que $\Lambda_{n} \longrightarrow \mathbb{Z}^{N}$. Usando la Afirmación para $\Lambda=A_{1}, B_{n}=\Lambda_{n}$ y $\Upsilon_{B_{n}}=\mu_{\Lambda_{n}}$. Por lo tanto, existe $B_{n}^{\Lambda}=$ $\left\{B_{n}^{A_{1}}\right\}_{n=1}^{\infty}$ subsucesión de $\left\{\Lambda_{n}\right\}$ tal que

$$
\lim _{n \rightarrow \infty} \mu_{B_{n}^{A_{1}}}\left(p_{A_{1} B_{n}^{A_{1}}}^{-1}\{\omega\}\right) \quad \text { existe } \forall \omega \in A_{1} .
$$

Paso 2. Usando nuevamente la Afirmación para $\Lambda=A_{2}, B_{n}=B_{n}^{A_{1}} y \Upsilon_{B_{n}}=\mu_{B_{n}^{A_{1}}}$. Por lo tanto, existe $\left\{B_{n}^{A_{2}}\right\}_{n=1}^{\infty}$ subsucesión de $\left\{B_{n}^{A_{1}}\right\}$ tal que

$$
\lim _{n \rightarrow \infty} \mu_{B_{n}^{A_{2}}}\left(p_{A_{2} B_{n}^{A_{2}}}^{-1}\{\omega\}\right) \quad \text { existe } \forall \omega \in A_{2} .
$$

Siguiendo así sucesivamente, aplicando la Afirmación para cada uno de los primeros $A_{k}$, para el Paso $k+1$ tenemos lo siguiente

Paso $k+1$. Finalmente usando la afirmación para $\Lambda=A_{k}, B_{n}=B_{n}^{A_{k-1}} y \Upsilon_{B_{n}}=\mu_{B_{n}}^{A_{k-1}}$. Por lo tanto, existe $\left\{B_{n}^{A_{k}}\right\}_{n=1}^{\infty}$ subsucesión de $\left\{B_{n}^{A_{k-1}}\right\}$ tal que

$$
\lim _{n \rightarrow \infty} \mu_{B_{n}^{A_{k}}}\left(p_{A_{k} B_{n}^{A_{k}}}^{-1}\{\omega\}\right) \quad \text { existe } \forall \omega \in A_{k} .
$$

Ahora nos vamos por la diagonal, es decir, sea $\Lambda_{n}^{\prime}=B_{n}^{A_{n}}$ la cual es una subsucesión de por lo menos la primera sucesión $\left\{B_{n}^{A_{1}}\right\}_{n \in \mathbb{N}}$, por lo tanto tenemos que $\Lambda_{n}^{\prime} \longrightarrow \mathbb{Z}^{N}$. Sea $\Lambda \in F$ por lo tanto existe $k \in \mathbb{N}$ tal que $\Lambda=A_{k}$. Si $\omega \in \Lambda$, entonces

$$
\begin{gathered}
\left\{\mu_{\Lambda_{n}^{\prime}}\left(p_{\Lambda \Lambda_{n}^{\prime}}^{-1}\{\omega\}\right)\right\}_{n=1}^{\infty} \text { es una subsucesión de }\left\{\mu_{B_{n}^{A_{k}}}\left(p_{\Lambda B_{n}^{A_{k}}}^{-1}\{\omega\}\right)\right\}_{n=1}^{\infty} \\
\text { por lo tanto } \lim _{n \rightarrow \infty} \mu_{\Lambda_{n}^{\prime}}\left(p_{\Lambda \Lambda_{n}^{\prime}}^{-1}\{\omega\}\right)=\lim _{n \rightarrow \infty} \mu_{B_{n}^{A_{k}}} p_{\Lambda B_{n}^{A_{k}}}^{-1}(\{\omega\})
\end{gathered}
$$


ii) Queremos mostrar la existencia de $\rho: \mathcal{C} \longrightarrow[0,1]$. Probaremos que $\left\{\rho_{\Lambda}: 2^{\Omega_{\Lambda}} \rightarrow\right.$ $[0,1] \mid \Lambda \in F\}$ es un sistema proyectivo de medidas de probabilidad, así por el teorema de Consistencia de Kolmogorov [4], existirá $\rho$ con las propiedades pedidas. Entonces sean $A \subset$ $B \subset \mathbb{Z}^{N}$ y $A, B \in F$. Mostraremos que

$$
\rho_{A}=\rho_{B} p_{A B}^{-1}
$$

lo que probará que $\left\{\rho_{\Lambda}: \Lambda \in F\right\}$ es un sistema proyectivo.

Puesto que para $\Lambda \in F$ cualquiera $\rho_{\Lambda}(H)=\sum_{\omega \in H} \rho_{\Lambda}(\omega), \forall H \subset \Omega_{\Lambda}$. Entonces

$$
\begin{aligned}
\rho_{\Lambda}\left(\Omega_{\Lambda}\right) & =\sum_{\omega \in \Omega_{\Lambda}} \rho_{\Lambda}(\omega)=\sum_{\omega \in \Omega_{\Lambda}} \lim _{k \rightarrow \infty} \mu_{\Lambda_{k}^{\prime}}\left(p_{\Lambda \Lambda_{k}^{\prime}}^{-1}\{\omega\}\right) \\
& =\lim _{k \rightarrow \infty} \sum_{\omega \in \Omega_{\Lambda}} \mu_{\Lambda_{k}^{\prime}}\left(p_{\Lambda \Lambda_{k}^{\prime}}^{-1}\{\omega\}\right)=\lim _{k \rightarrow \infty} \mu_{\Lambda_{k}^{\prime}}\left(\bigcup_{\omega \in \Omega_{\Lambda}} p_{\Lambda \Lambda_{k}^{\prime}}^{-1}\{\omega\}\right) \\
& =\lim _{k \rightarrow \infty} \mu_{\Lambda_{k}^{\prime}}\left(\Omega_{\Lambda_{k}^{\prime}}\right)=1 .
\end{aligned}
$$

Por lo tanto tenemos que $\rho_{\Lambda}: 2^{\Omega_{\Lambda}} \longrightarrow[0,1]$ es medida de probabilidad. Así, nos queda mostrar que $\rho_{A}\{H\}=\rho_{B}\left\{p_{A B}^{-1}\{H\}\right\}, \forall H \in 2^{\Omega_{A}}$. Pero basta con tomar $H=\{\omega\}$ con $\omega \in A$. Asi pues, por demostrar que:

$$
\rho_{A}\{\omega\}=\rho_{B}\left\{p_{A B}^{-1}\{\omega\}\right\} \quad \forall \omega \in \Omega_{A}
$$

Notemos que

$$
\begin{aligned}
\rho_{B}\left\{p_{A B}^{-1}(\omega)\right\} & =\rho_{B}\left(\left\{\omega^{\prime} \in \Omega_{B}:\left.\omega^{\prime}\right|_{A}=\omega\right\}\right)=\sum_{\omega^{\prime} \in p_{A B}^{-1}(\omega)} \rho_{B}\left(\omega^{\prime}\right) \\
& =\sum_{\omega^{\prime} \in p_{A B}^{-1}(\omega)} \lim _{n \rightarrow \infty} \mu_{\Lambda_{n}^{\prime}}\left(p_{B \Lambda_{n}^{\prime}}^{-1}\left(\omega^{\prime}\right)\right) \\
& =\lim _{n \rightarrow \infty} \sum_{\omega^{\prime} \in p_{A B}^{-1}(\omega)} \mu_{\Lambda_{n}^{\prime}}\left(p_{B \Lambda_{n}^{\prime}}^{-1}\left(\omega^{\prime}\right)\right) \\
& =\lim _{n \rightarrow \infty} \sum_{\omega^{\prime} \in p_{A B}^{-1}(\omega)} \sum_{\omega^{\prime \prime} \in p_{B \Lambda_{n}^{-1}}\left(\omega^{\prime}\right)} \mu_{\Lambda_{n}^{\prime}}\left(\omega^{\prime \prime}\right)
\end{aligned}
$$

Ahora observemos que $B \subset \Lambda_{n}^{\prime}, p_{B \Lambda_{n}^{\prime}}: \Omega_{\Lambda_{n}^{\prime}} \longrightarrow \Omega_{B}, p_{A B}: \Omega_{B} \longrightarrow \Omega_{A}$ y $p_{A \Lambda_{n}^{\prime}}: \Omega_{\Lambda_{n}^{\prime}} \longrightarrow \Omega_{A}$, 
entonces la ecuación $(4,2)$ es:

$$
\begin{aligned}
\rho_{B}\left\{p_{A B}^{-1}(\omega)\right\} & =\lim _{n \rightarrow \infty} \sum_{\omega^{\prime} \in p_{A B}^{-1}(\omega)} \sum_{\omega^{\prime \prime} \in p_{B \Lambda_{n}^{-1}}^{\prime}\left(\omega^{\prime}\right)} \mu_{\Lambda_{n}^{\prime}}\left(\omega^{\prime \prime}\right) \\
& =\lim _{n \rightarrow \infty} \mu_{\Lambda_{n}^{\prime}}\left(\bigcup_{\omega^{\prime} \in p_{A B}^{-1}(\omega)} \bigcup_{\omega^{\prime \prime} \in p_{B \Lambda_{n}^{\prime}}^{-1}\left(\omega^{\prime}\right)}\left\{\omega^{\prime \prime}\right\}\right) \\
& =\lim _{n \rightarrow \infty} \mu_{\Lambda_{n}^{\prime}}\left(p_{A \Lambda_{n}^{\prime}}^{-1}(\omega)\right) \\
& =\rho_{A}(\omega) .
\end{aligned}
$$

En [8] se da una prueba del teorema anterior con ayuda de otro teorema, el Teorema de Stone-Weirstrass.

Sea $\phi$ una interacción dada, $U_{\Lambda}^{\phi}$ función de energía y $W_{\Lambda \Lambda^{c}}^{\phi}$ la interacción entre los subconjuntos $\Lambda$ y $\Lambda^{c}$, con esto tenemos la definición de estado de Gibbs.

Sea $\Lambda \in \mathcal{F}, \omega=\left(\omega_{1}, \omega_{2}\right)$ y $\eta=\left(\alpha, \omega_{2}\right)=\eta \omega_{2}$. Tenemos que

$$
\mu\left(p_{\Lambda}^{-1}\left(\omega_{1}\right) \mid p_{\Lambda^{c}}^{-1}\left(\omega_{2}\right)\right)=\frac{e^{-U_{\Lambda}^{\phi}\left(\omega_{1}\right)-W_{\Lambda \Lambda^{c}}^{\phi}(\omega)}}{\sum_{\alpha \in \Omega_{\Lambda}} e^{-U_{\Lambda}^{\phi}(\alpha)-W_{\Lambda \Lambda^{c}}^{\phi}\left(\alpha \omega_{2}\right)}} .
$$

Observemos que $\mu$ en 4.3 es la probabilidad condicional regular de $p_{\Lambda}^{-1}\left(\omega_{1}\right)$ dado $p_{\Lambda^{c}}^{-1}\left(\omega_{2}\right)$.

Tenemos la siguiente convención, si el término del estado de Gibbs $e^{-W_{\Lambda \Lambda}^{\Phi}(\omega)}=0$ entonces el estado de Gibbs será igual a cero lo cual no es medida de probabilidad.

Definición 45. (Estado de Gibbs). Decimos que $\sigma$ medida de probabilidad es un estado de Gibbs para la interacción $\phi$ si, para todo $\Lambda \subset \mathcal{F}$, existe una medida de probabilidad $\sigma_{\Lambda^{c}}: \mathcal{C}\left(\Omega_{\Lambda^{c}}\right) \longrightarrow[0,1]$ tal que para todo $\omega_{1} \in \Omega_{\Lambda}$

$$
p_{\Lambda}\left(\sigma\left(\omega_{1}\right)\right)=\int_{\Omega_{\Lambda^{c}}} \sigma_{\Lambda}\left(d \omega_{2}\right) \mu\left(p_{\Lambda}^{-1}\left(\omega_{1}\right) \mid p_{\Lambda^{c}}^{-1}\left(\omega_{2}\right)\right)
$$

donde $\mu\left(p_{\Lambda}^{-1}\left(\omega_{1}\right) \mid p_{\Lambda^{c}}^{-1}\left(\omega_{2}\right)\right)$ es como en 4.3.

Teorema 34. El límite termodinámico de ensambles de Gibbs es un estado de Gibbs. 
Demostración. Ver [8] pp.15-16.

Teorema 35. Una medida de probabilidad $\sigma: \Omega \longrightarrow[0,1]$ es un estado de Gibbs si y sólo si, para cada $\Lambda \in \mathcal{F}$, la probabilidad condicional que $\left.\omega\right|_{\Lambda}=\omega_{1}$, sabiendo que $\left.\omega\right|_{\Lambda^{c}}=\omega_{2}$ es $\mu\left(p_{\Lambda}^{-1}\left(\omega_{1}\right) \mid p_{\Lambda^{c}}^{-1}\left(\omega_{2}\right)\right)$ como en 4.3 .

Demostración. Ver [8] pp. 18-19.

El Teorema 35 se considera como definición de estado de Gibbs en [9]. Finalmente decimos que un estado es invariante si éste es invariante bajo la traslación $\Psi^{n}$ con $n \in \mathbb{Z}$. Así, sea $\chi$ el conjunto de todos los estados invariantes en $\Omega$.

Definición 46. (Entropía media). Si $\mu$ es un estado invariante sobre $\Omega$, la entropía media de $\mu$, denotada por $S(\mu)$, se define por

$$
S(\mu)=\lim _{n \rightarrow \infty} \frac{1}{\left|\Lambda_{n}\right|} S\left(P_{\Lambda_{n}}\right)
$$

con $\Lambda_{n}=\{0, \ldots, n-1\}^{N}$ y $S\left(P_{\Lambda_{n}}\right)$ la entropía de las distribuciones finito dimensionales $P_{\Lambda}$.

Definición 47. (Presión). Sea ф una interacción. La presión de $\phi$, está definida por

$$
P(\phi)=\lim _{n \rightarrow \infty} \frac{1}{\left|\Lambda_{n}\right|} \log Z_{\Lambda_{n}}(\phi)
$$

con $Z_{\Lambda_{n}}$ la función partición de la interacción.

Definición 48. (Energía de la interacción). Dada una interacción $\phi$ y un estado invariante $\mu$. La energía de la interacción $\phi$ para el estado $\mu$, se define como

$$
\mu(\phi)=-\lim _{n \rightarrow \infty} \frac{1}{\left|\Lambda_{n}\right|} \sum_{\omega \in \Omega_{\Lambda_{n}}} P_{\Lambda_{n}}(\omega) U_{\Lambda_{n}}^{\phi}(\omega)
$$

Con estas definiciones tenemos el siguiente Teorema:

Teorema 36. Para cualquier interacción $\phi$,

$$
\begin{aligned}
P(\phi) & =\operatorname{máx}\{S(\mu)-\mu(\phi): \mu \in \chi\} \\
& =\operatorname{mín}\{-(S(\mu)-\mu(\phi)): \mu \in \chi\}
\end{aligned}
$$

Demostración. Ver [8]. 
Como podemos observar, este teorema es análogo al principio variacional en sistemas lattice finitos.

Siguiendo [9] y [8] tenemos los siguientes resultados: Un estado de equlibrio para una interacción $\phi$ es cualquier estado invariante donde se obtiene el máximo en el Teorema 35. El resultado fundamental es que dada una interacción $\phi$ sobre $\Omega$, un estado invariante es un estado de equilibrio para $\phi$ si y sólo si éste es un estado de Gibbs dada $\phi$.

Así, con la teoría tanto del capítulo III como de éste podemos dar las siguientes relaciones.

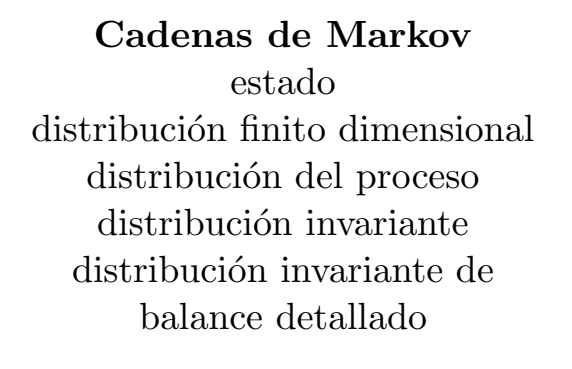

Cadenas de Markov

estado
distribución finito dimensional
distribución del proceso
distribución invariante
distribución invariante de
balance detallado

$\begin{array}{cc} & \text { Sistemas Clásicos Lattice } \\ \longrightarrow & \text { símbolo ó microestado } \\ \longrightarrow & \text { ensamble canónico } \\ \longrightarrow & \text { límite termodinámico } \\ \longrightarrow & \text { estado invariante } \\ & \\ \longrightarrow & \text { ensamble de Gibbs }\end{array}$

Si consideramos una cadena de Markov estacionaria $\left\{X_{n}\right\}_{n \in \mathbb{Z}}$, la lattice del sistema es $\mathbb{Z}$,

\section{Sistema lattice $\mathbb{Z}$}

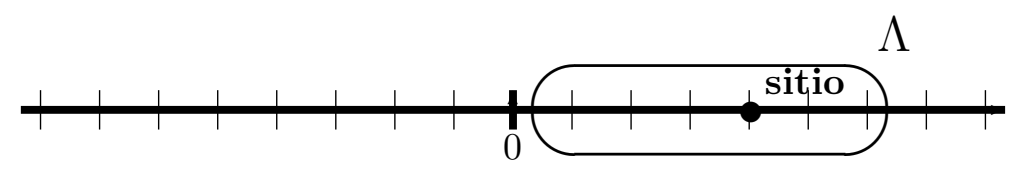

Donde $T=\mathbb{Z}$ y $(S, \mathfrak{S})$ un espacio medible con $S=\Omega_{0}=\{0,1,2, \ldots\}$ y $\mathfrak{S}=2^{S}$. El espacio de configuraciones del sistema (cadena) es $\Omega=\Omega_{0}^{T}$. Sea $\Lambda \subset T$ como se observa en la figura anterior, $a \in \Lambda$ un sitio. Una configuración que denotaremos por $X_{\omega}(\cdot): \mathbb{Z} \rightarrow \Omega_{0}$ la cual es una trayectoria, en teoría de cadenas de Markov, toma valores en $\Omega_{0}$ el cual es llamado espacio de microestados para estas cadenas.

Un estado es cualquier medida de probabilidad $\mu: \mathcal{C}\left(\Omega_{\Lambda}\right) \rightarrow[0,1]$ por lo tanto si $\omega \in \Omega_{\Lambda}$ entonces $\mu(\omega)$ es la probabilidad de que se presenten los microestados $\omega(k)$ con $k$ sitios en $\Lambda$. 
Esta distribución de probabilidad describe lo que pasa en la configuración dada y el conjunto de todas las distribuciones de probabilidad o bien de los estados, describe lo que pasa en todo el sistema lattice [22].

La función de interacción $\Phi: \bar{\Omega} \rightarrow \mathbb{R}$ se puede pensar como el cambio de información entre los sitios y la función de energía $U_{\Lambda}^{\Phi}: \Omega_{\Lambda} \rightarrow \mathbb{R}$ es la energía potencial del sistema que puede pensarse como la energía almacenada en el sistema [22].

Finalmente el ensamble de Gibbs dada la interacción $\Phi$ es la distribución de probabilidad $P_{\Lambda}: \Omega_{\Lambda} \rightarrow \mathbb{R}$ tal que

$$
P_{\Lambda}\left(\omega_{0}\right)=\frac{e^{-U_{\Lambda}^{\Phi}}}{\sum_{\omega \in \Omega_{\Lambda}} e^{-U_{\Lambda}^{\Phi}(\omega)}}
$$

donde como se puede observar a menor energía, la configuración $\omega_{0}$ es más probable.

Un lattice en $\mathbb{Z}^{2}$ se ve de la siguiente forma:

\section{Sistema lattice $\mathbb{Z}^{2}$}

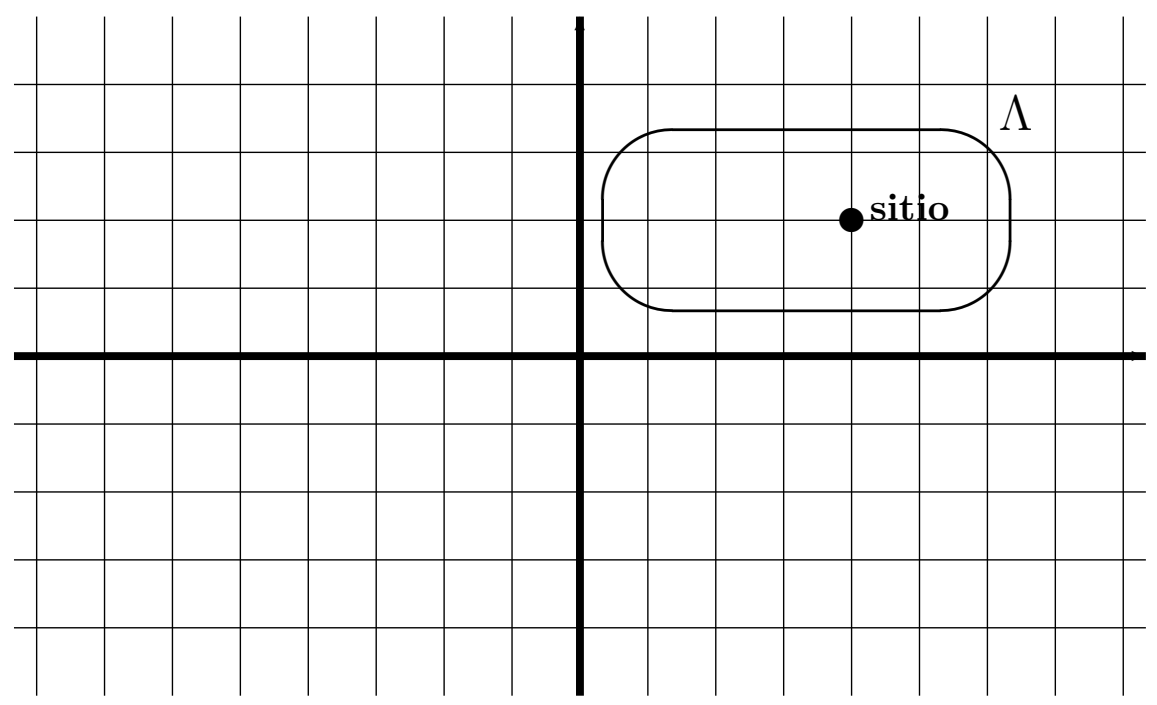

En este lattice se modela un gas que contiene $k$ sitios. Los estados del sistema al tiempo $t$ son especifícados por el conjunto de microestados $\left\{n\left(c_{k}, r, t\right)\right\}$ donde $n\left(c_{k}, r, t\right)$ es uno si hay una partícula en el sitio $r$ con velocidad $c_{k}$ y 0 en otro caso.

Proponemos algunos ejemplos para interacciones específicas. 
Ejemplo 24. Sea $\Omega_{0}=\{0,1, \ldots, k\}, \Omega=\Omega_{0}^{T}, \Omega_{\Lambda}=\left\{\omega \mid \omega: \Lambda \rightarrow \Omega_{0}\right\}$. Definamos la interacción para todo $\omega \in \Omega$ tal que $\Phi(\omega) \equiv 0$. Verifiquemos que es interacción:

1. $\Phi \mid(\emptyset)=0$

2. $\Phi\left(T_{a} \omega\right)=0=\Phi(\omega)$ con $a \in T$.

3. $|\Phi|_{x}=\sum_{\Lambda \ni x} \frac{1}{|\Lambda|} \sup _{\omega \in \Omega_{\Lambda}}|\Phi(\omega)|=\sum_{\Lambda \ni x} \frac{1}{|\Lambda|} 0=0<+\infty$.

La función de energía dada la interacción $\Phi$ con $\Lambda \in \mathcal{F}$ es:

$$
U_{\Lambda}^{\Phi}(\omega)=\sum_{\Lambda^{\prime} \subset \Lambda} \Phi\left(\left.\omega\right|_{\Lambda^{\prime}}\right)=\sum_{\Lambda^{\prime} \subset \Lambda} 0=0
$$

y además

$$
\|\Phi\|_{x}=\sum_{\Lambda \ni x} \sup _{\omega \in \Omega_{\Lambda}}|\Phi(\omega)|=0<+\infty .
$$

Por tanto podemos calcular $W_{\Lambda \Lambda^{c}}^{\Phi}$ tal que

$$
W_{\Lambda \Lambda^{c}}^{\Phi}(\omega)=\sum_{\substack{\Lambda^{\prime}, \Lambda_{1} \cup \Lambda_{2} \\ \Lambda^{\prime} \cap \Lambda_{1} \neq \emptyset \\ \Lambda^{\prime} \cap \Lambda_{2} \neq \emptyset}} \Phi\left(\left.\omega\right|_{\Lambda^{\prime}}\right)=\sum_{\substack{\Lambda^{\prime} \subset \Lambda_{1} \cup \Lambda_{2} \\ \Lambda^{\prime} \cap \Lambda_{1} \neq \emptyset \\ \Lambda^{\prime} \cap \Lambda_{2} \neq \emptyset}} 0=0 .
$$

Así, las probabilidades condicionales del estado de Gibbs asociado a esta interacción es

$$
\begin{aligned}
\mu\left(P_{\Lambda}^{-1}\left(\omega_{1}\right) \mid P_{\Lambda^{c}}^{-1}\left(\omega_{2}\right)\right) & =\frac{e^{-U_{\Lambda}^{\Phi}\left(\omega_{1}\right)-W_{\Lambda \Lambda^{c}}^{\Phi}(\omega)}}{\sum_{\alpha \in \Omega_{\Lambda}} e^{-U_{\Lambda}^{\Phi}(\alpha)-W_{\Lambda \Lambda^{c}}^{\Phi}\left(\alpha \omega_{2}\right)}} \\
& =\frac{e^{-0-0}}{\sum_{\alpha \in \Omega_{\Lambda}} e^{-0-0}}=\frac{e^{0}}{\sum_{\alpha \in \Omega_{\Lambda}} e^{0}} \\
& =\frac{1}{\sum_{\alpha \in \Omega_{\Lambda}} 1}=\frac{1}{\left|\Omega_{\Lambda}\right|}=\frac{1}{(k+1)^{|\Lambda|}}
\end{aligned}
$$

Por lo tanto de acuerdo a la Definición 45, el estado de Gibbs $\sigma$ asociado a la interacción $\Phi$ debe satisfacer que

$$
p_{\Lambda}\left(\sigma\left(\omega_{1}\right)=\int_{\Omega_{\Lambda^{c}}} \sigma_{\Lambda}\left(d \omega_{2}\right) \frac{1}{(k+1)^{|\Lambda|}}=\frac{1}{(k+1)^{|\Lambda|}} \int_{\Omega_{\Lambda^{c}}} \sigma_{\Lambda}\left(d \omega_{2}\right)=\frac{1}{(k+1)^{|\Lambda|}} .\right.
$$

que son las distribuciones finito dimensionales de variables aleatorias independientes y distribuidas uniformemente. 
Ejemplo 25. Sea $\Omega_{0}=\{0,1, \ldots, k\}, \Omega=\Omega_{0}^{T}, \Omega_{\Lambda}=\left\{\omega \mid \omega: \Lambda \rightarrow \Omega_{0}\right\}$. Definimos la siguiente interacción

$$
\Phi\left(\left.\omega\right|_{\Lambda}\right)=\left\{\begin{array}{cc}
1 & \text { si }|\Lambda|=1 \\
0 & \text { en otro caso }
\end{array}\right.
$$

Tenemos que ésta función definida así cumple que

1. $\Phi\left(\left.\omega\right|_{\emptyset}\right)=0$ por definición.

2. $\Phi\left(\left.T_{a} \omega\right|_{\Lambda}\right)=\left\{\begin{array}{cc}1 & \text { si }|\Lambda|=1 \\ 0 & \text { en otro caso }\end{array}=\Phi\left(\left.\omega\right|_{\Lambda}\right)\right.$.

Si suponemos que $\|\Phi\|_{x}<\infty$ entonces al calcular la función de energía tenemos que

$$
U_{\Lambda}^{\Phi}(\omega)=\sum_{\Lambda^{\prime} \subset \Lambda} \Phi\left(\left.\omega\right|_{\Lambda^{\prime}}\right)
$$

Puesto que $\Lambda$ es finito, entonces el número de subconjuntos de $\Lambda$ tales que $\Lambda^{\prime} \subset \Lambda$ con $\left|\Lambda^{\prime}\right|=1$ es igual $|\Lambda|$ y para subconjuntos de $\Lambda$ con cardinalidad mayor que uno la interacción $\Phi$ es cero, por lo tanto

$$
U_{\Lambda}^{\Phi}(\omega)=\sum_{\Lambda^{\prime} \subset \Lambda:\left|\Lambda^{\prime}\right|=1} 1=|\Lambda|
$$

$Y$ ya que no existe algun conjunto $\Lambda^{\prime}$ con un elemento tal que $\Lambda^{\prime} \cap \Lambda \neq \emptyset$ y $\Lambda^{\prime} \cap \Lambda^{c} \neq \emptyset$, entonces $W_{\Lambda \Lambda^{c}}^{\Phi}(\omega)=0$. Finalmente, las probabilidades condicionales del estado Gibbsiano asociado a la interacción $\Phi$ es

$$
\begin{aligned}
\mu\left(P_{\Lambda}^{-1}\left(\omega_{1}\right) \mid P_{\Lambda^{c}}^{-1}\left(\omega_{2}\right)\right) & =\frac{e^{-U_{\Lambda}^{\Phi}\left(\omega_{1}\right)-W_{\Lambda \Lambda^{c}}^{\Phi}(\omega)}}{\sum_{\alpha \in \Omega_{\Lambda}} e^{-U_{\Lambda}^{\Phi}(\alpha)-W_{\Lambda \Lambda^{c}}^{\Phi}\left(\alpha \omega_{2}\right)}}=\frac{e^{-|\Lambda|}}{\sum_{\alpha \in \Omega_{\Lambda}} e^{-|\Lambda|}} \\
& =\frac{1}{\sum_{\alpha \in \Omega_{\Lambda}} 1}=\frac{1}{(k+1)^{|\Lambda|}}
\end{aligned}
$$

Donde el estado de Gibbs $\sigma$ asociado a $\Phi$ satisface que

$$
p_{\Lambda}\left(\sigma\left(\omega_{1}\right)=\int_{\Omega_{\Lambda^{c}}} \sigma_{\Lambda}\left(d \omega_{2}\right) \frac{1}{(k+1)^{|\Lambda|}}=\frac{1}{(k+1)^{|\Lambda|}} \int_{\Omega_{\Lambda^{c}}} \sigma_{\Lambda}\left(d \omega_{2}\right)=\frac{1}{(k+1)^{|\Lambda|}} .\right.
$$

donde nuevamente son las distribuciones finito dimensionales de variables aleatorias independientes y distribuidas uniformemente.

Ejemplo 26. Sea $\Omega_{0}=\{0,1, \ldots, k\}, \Omega=\Omega_{0}^{T}, \Omega_{\Lambda}=\left\{\omega \mid \omega: \Lambda \rightarrow \Omega_{0}\right\}$. Sea la interacción $\Phi$ tal que

$$
\Phi\left(\left.\omega\right|_{\Lambda}\right)=\left\{\begin{array}{cc}
1 & \text { si }|\Lambda| \leq 2 \\
0 & \text { si }|\Lambda| \geq 3 y|\Lambda|=0
\end{array}\right.
$$

Nuevamente tenemos que: 
1. $\Phi\left(\left.\omega\right|_{\emptyset}\right)=0$ por definición.

2. $\Phi\left(T_{a} \omega\right)=\left\{\begin{array}{cc}1 & \text { si }|\Lambda| \leq 2 \\ 0 & \text { si }|\Lambda| \geq 3 y|\Lambda|=0\end{array}=\Phi(\omega)\right.$ con $a \in \mathbb{Z}^{N}$.

Supongamos que $|\Phi|_{x}<+\infty$. Calculando la función de energía tenemos que

$$
\begin{aligned}
U_{\Lambda}^{\Phi}(\omega) & =\sum_{\Lambda^{\prime} \subset \Lambda} \Phi\left(\left.\omega\right|_{\Lambda^{\prime}}\right) \\
& =\sum_{\Lambda^{\prime} \subset \Lambda:\left|\Lambda^{\prime}\right|=1} \Phi\left(\left.\omega\right|_{\Lambda^{\prime}}\right)+\sum_{\Lambda^{\prime} \subset \Lambda:\left|\Lambda^{\prime}\right|=2} \Phi\left(\left.\omega\right|_{\Lambda^{\prime}}\right)+\sum_{\Lambda^{\prime} \subset \Lambda:\left|\Lambda^{\prime}\right| \geq 3} \Phi\left(\left.\omega\right|_{\Lambda^{\prime}}\right) \\
& =|\Lambda|+\left(\begin{array}{c}
|\Lambda| \\
2
\end{array}\right)+0=|\Lambda|+\frac{|\Lambda|(|\Lambda|-1)}{2}=|\Lambda|\left(\frac{|\Lambda|+1}{2}\right)
\end{aligned}
$$

Para calcular $W_{\Lambda \Lambda^{c}}^{\Phi}$ consideremos sólo los subconjuntos de $\Lambda$ tales que $|\Lambda|=2$ y nos interesan los subconjuntos de $\Lambda$ tales que $\Lambda^{\prime}=\{a, b\}$ con $a \in \Lambda$ y $b \in \Lambda^{c}$, así

$$
W_{\Lambda \Lambda^{c}}^{\Phi}(\omega)=\sum_{\Lambda^{\prime} \subset \mathbb{Z}^{N}: \Lambda^{\prime}=\{a, b\}} \Phi\left(\left.\omega\right|_{\Lambda^{\prime}}\right)=\sum_{\Lambda^{\prime} \subset \mathbb{Z}^{N}: \Lambda^{\prime}=\{a, b\}} 1=+\infty
$$

Por lo tanto las probabilidades condicionales del estado de Gibbs asociado a ésta interacción es cero, lo cual no es medida de probabilidad, es decir, para ésta interacción no hay estado de Gibbs.

Ejemplo 27. Sea $\Omega_{0}=\{0,1, \ldots, k\}, \Omega=\Omega_{0}^{\mathbb{Z}^{2}}, \Omega_{\Lambda}=\left\{\omega \mid \omega: \Lambda \rightarrow \Omega_{0}\right\}$. Consideremos la interacción definida por

$$
\Phi\left(\left.\omega\right|_{\Lambda}\right)=\left\{\begin{array}{cc}
1 & \text { si diam } \Lambda=1 \\
0 & \text { sidiam } \Lambda>1 \text { ó } \Lambda=\emptyset
\end{array}\right.
$$

Nuevamente ésta interacción es invariante bajo traslaciones pues el diametro de $\Lambda$ es invariante bajo traslaciones y $\Phi\left(\left.\omega\right|_{\emptyset}\right)=0$ por definición. Los subconjuntos de $\mathbb{Z}^{2}$ tales que diam $\Lambda=1$ tiene cuando mucho 4 elementos y $|\Phi|_{x}=\sum_{\Lambda \ni x} \frac{1}{|\Lambda|} \cdot 1=\frac{8}{2}+\frac{8}{3}+\frac{4}{4}<+\infty$, la función de energía asociada a ésta interacción es

$$
U_{\Lambda}^{\Phi}(\omega)=\sum_{\Lambda^{\prime} \subset \Lambda} \Phi\left(\left.\omega\right|_{\Lambda^{\prime}}\right)=\sum_{\Lambda^{\prime} \subset \Lambda: \text { diam } \Lambda^{\prime}=1} 1=\left(\begin{array}{c}
|\Lambda| \\
2
\end{array}\right)+\left(\begin{array}{c}
|\Lambda| \\
3
\end{array}\right)+\left(\begin{array}{c}
|\Lambda| \\
4
\end{array}\right)
$$

Pero para $W_{\Lambda \Lambda^{c}}^{\Phi}$ tenemos el mismo caso que el ejemplo anterior, es decir, $W_{\Lambda \Lambda^{c}}^{\Phi}=+\infty$ pues

$$
\begin{aligned}
W_{\Lambda \Lambda^{c}}^{\Phi}(\omega) & =\sum_{\substack{\Lambda^{\prime} \subset \mathbb{Z}^{2} \\
\Lambda^{\prime} \cap \Lambda \neq \emptyset \\
\Lambda^{\prime} \cap \Lambda^{c} \neq \emptyset}} \Phi\left(\left.\omega\right|_{\Lambda^{\prime}}\right) \\
& =\left(\begin{array}{c}
\left|\mathbb{Z}^{2}\right| \\
2
\end{array}\right)+\left(\begin{array}{c}
\left|\mathbb{Z}^{2}\right| \\
3
\end{array}\right)+\left(\begin{array}{c}
\left|\mathbb{Z}^{2}\right| \\
4
\end{array}\right) \\
& =+\infty
\end{aligned}
$$


por lo tanto para esta interacción las probabilidades condicionales del estado de Gibbs es cero el cual no es medida de probabilidad y por lo tanto no hay estado de Gibbs y no hay equilibrio.

Pero ¿que relación hay entre los estados de Gibbs y el balance detallado?. Considerando cadenas de Markov irreducibles con $\left\{\pi_{x}\right\}_{x=1}^{N}$ distribución invariante estrictamente positiva, siguiendo a [13] define la tasa de producción de entropía como $E_{p}$ :

$$
E_{p}=\frac{1}{2} \sum_{i, j}\left(\pi_{x} p(x, y)-\pi_{y} p(y, x)\right) \log \frac{\pi_{x} p(x, y)}{\pi_{y} p(y, x)}
$$

para todo $p(x, y)>0$ con $x, y \in S$, donde, como se puede observar, el proceso está en balance detallado si y sólo si la producción de entropía $E_{p}=0$. Como bien se menciona en [13], un sistema que no está en equilibrio es un sistema estacionario abierto con tasa de producción de entropía positiva, es decir, intercambia sustancias y energía con su medio ambiente. Lo que nos impone la condición de balance detallado es que, en media, el sistema pasa tantas veces del estado $x$ al estado $y$ como del estado $y$ al estado $x$, lo cual tiene una ventaja física. La mayoria de los sistemas que interesa simular siguen las leyes de la mécanica clásica o cuántica, las cuales son invariantes bajo traslaciones del tiempo. Con la condición de balance detallado, una simulación también lo es, siendo así una representación más adecuada de lo que se observa en la naturaleza.

Por lo tanto cualquier proceso de Markov irreducible que construyamos cuyas probabilidades de transición cumplan con la condición de balance detallado nos asegura que la distribución de equilibrio de la cadena de Markov generada será el ensamble de Gibbs como se puede ver en [9] pp.241-244.

Un ejemplo de la segunda equivalencia se da en [13], donde dada una cadena de Markov estacionaria que modela la combinación y transformación de polímeros bioquímicos, su distribución invariante está dada por el ensamble de Gibbs

$$
\pi_{x}=\frac{e^{-F_{x} / k T}}{\sum_{y} e^{-F_{y} / k T}}
$$

con $x, y \in S$ y $\beta=(k T)^{-1}$ constante de Boltzmann, y bajo la condición de balance detallado, $F_{x}$ es la energía libre del sistema en el estado $x$. Además tenemos el ensamble de Gibbs para ciertas cadenas de Nacimiento y Muerte.

Ejemplo 28. Consideremos nuevamente una cadena de Nacimiento y Muerte con intensidades definidas de la siguiente manera, $\lambda_{x}=(x+1)^{n}$ y $\mu_{x}=x^{n}$ con $x \in S, n \in \mathbb{N}$. La 
distribución invariante es

$$
\pi(x)=\frac{\Pi_{y=0}^{x} \frac{\lambda_{y}}{\mu_{y+1}}}{\sum_{x=0}^{\infty} \Pi_{y=0}^{x} \frac{\lambda_{y}}{\mu_{y+1}}}=\frac{\Pi_{y=0}^{x} \frac{\lambda(y+1)}{\mu(y+1)}}{\sum_{x=0}^{\infty} \Pi_{y=0}^{x} \frac{\lambda(y+1)}{\mu(y+1)}}=\frac{\Pi_{y=0}^{x} \frac{\lambda}{\mu}}{\sum_{x=0}^{\infty} \Pi_{y=0}^{x} \frac{\lambda}{\mu}}=\frac{\left(\frac{\lambda}{\mu}\right)^{x+1}}{\sum_{x=0}^{\infty}\left(\frac{\lambda}{\mu}\right)^{x+1}}
$$

donde la serie del denominador converge si $\mu>\lambda$ y en este caso la cadena está en balance detallado. El ensamble de Gibbs es

$$
\pi(x)=\frac{e^{-\sum_{y=0}^{x} \log \left(\frac{\lambda_{y}}{\mu_{y+1}}\right)^{-1}}}{\sum_{z=0}^{\infty} e^{-\sum_{y=0}^{z} \log \left(\frac{\lambda_{y}}{\mu_{y}+1}\right)^{-1}}}
$$

Observamos que la función $U_{\Lambda}^{\phi}=\sum_{y=0}^{x} \log \left(\frac{\lambda_{y}}{\mu_{y+1}}\right)^{-1}$ es la función de energía dada una interacción $\phi$. Así, el ensamble de Gibbs es

$$
\pi(x)=\frac{e^{-\sum_{y=0}^{x} \log \left(\frac{\lambda_{y}}{\mu_{y+1}}\right)^{-1}}}{\sum_{z=0}^{\infty} e^{-\sum_{y=0}^{z} \log \left(\frac{\lambda_{y}}{\mu_{y+1}}\right)^{-1}}}=\frac{e^{-\sum_{y=0}^{x} \phi(y)}}{\sum_{z=0}^{\infty} e^{-\sum_{y=0}^{z} \phi(y)}}=\frac{e^{-U_{\Lambda}^{\phi}(x)}}{\sum_{z=0}^{\infty} e^{-U_{\Lambda}^{\phi}(z)}} .
$$




\section{Conclusiones y Perspectivas}

Al hacer un análisis de las distintas nociones de equilibrio para procesos de Markov, obtuvimos un resultado para cadenas de Nacimiento y Muerte, donde estas cadenas tendrán distribución invariante si y sólo si está en balance detallado.

Se dio una prueba de la existencia del límite termodinámico con la ayuda del teorema de consistencia de Kolmogorov, a diferencia de la prueba usual que emplea el Teorema de representación de Riezs para funcionales positivas sobre el espacio de funciones continuas definidas sobre un espacio compacto.

Intentamos demostrar la afirmación en [13] que afirma que balance detallado es equivalente a la noción de equilibrio dada por D. Ruelle. Los ejemplos que desarrollamos ilustran que es perfectamente factible pero no logramos dar una prueba ni encontramos alguna en la literatura.

Finalmente construimos un ejemplo de un sistema fuera de equilibrio, con un número infinito de estados. 


\section{Bibliografía}

[1] C. Santisteban Requena, Modelos estocásticos de aprendizaje en ensayos de respuesta dicotómica y un número finito de estados absorbentes. Psicothema. Universidad de Oviedo, España, (2000), 519-521.

[2] R. Durret, Probability: Theory and examples. Wadsworth, Inc. Belmont, California, (1991).

[3] J. Lamperti, Stochastics processes. Dartmouth College. Hanover, New Hamshire (1977).

[4] C. Tudor, Procesos Estocásticos, Tercera edición. Textos 2, nivel avanzado. Sociedad Matemática Mexicana, (2002).

[5] L. Olivares, L. García Colín, Plegamiento de las proteínas: Un problema interdisciplinario, Universidad Autónoma Metropolitana-Iztapalapa, Rev. Soc Quím. Méx. 2004, 48, 95-105.

[6] L. Olivares, Tesis: El modelo extendido de Zwanzing y la teoría de Adam-Gibbs en el plegamiento y desnaturalización de proteínas, (2007).

[7] W. Rudin, Principios de análisis matemático, McGraw Hill, 3a edición (1980).

[8] D. Ruelle, Thermodynamic Formalism, The mathematical structures of equilibrium, Second edition (2004).

[9] J. England, F.G. Martin, Mathematical theory of entropy, Addison - Wesley Publishing Company, Volumen 12, (1981).

[10] D. Bryngelson, G. Wolynes, Spin glasses and the statistical mechanics of protein folding, Noyes Laboratory, University of Illinois, Communicated by Hans Frauenfelder, (1987). 
[11] W. Rudin, Análisis real y complejo, McGraw Hill, (1985).

[12] L. Rincón, Introducción a los Procesos Estocásticos, Departamento de Matemáticas, Facultad de Ciencias UNAM.

[13] J. Da-Quan, M. Qian, M-P. Qian, Mathematical Theory of Nonequilibrium Steady States, On the Frontier of Probability and Dynamical Systems, Springer, August 16, (2003).

[14] S. Ethier, T. Kurtz, Markov Processes, Characterization and Convergence, Wiley-Interscience, (1986) by John Wiley \& Sons, (2005).

[15] R. Munkres, Topology, 2nd Edition, Massachusetts Institute of Technology, (2000), by Prentice Hall, Inc, (1975).

[16] V. Tkachuk, Curso básico de topología general, Universidad Autónoma Metropolitana, Libros de Texto, (1999).

[17] S. Brassesco, Cadenas de Markov, Departamento de Matemáticas, Instituto Venezolano de Investigaciones Científicas.

[18] D. Bricio, Caminatas Aleatorias y Movimiento Browniano, Monografías del instituto de matemáticas, UNAM, (1981).

[19] K. Chung, Markov Chains with stationary transition probabilities, SpringerVerlag- Berlin-Heidelberg, (1960).

[20] L. Breiman, Probabilidad, In Applied Mathematics, University of California, Berkeley, Sociedad for Industrial ans Applied Mathematics, Philadelphia, (1992).

[21] G. Hoel, C. Port and J. Stone, Introduction to Stochastic Processes, University of California, Los Angeles, Houghton Mifflin Company, Boston, (1972).

[22] D. Ruelle, Statistical Mechanics, Rigorous Results, Imperial College Press and World Scientific Publishing, (1999). 
Algunas nociones de equilibrio en sistemas estocásticos

Tesis que presenta:

Mat. Lizeth Marianita Hernández Hernández

Para obtener el grado de:

Maestro en Ciencias

Matemáticas Aplicadas e Industriales

Poserado en Matemáticas

Departamento de Matemáticas

Universidad Avtonóma Metropolitana

trtapalapa

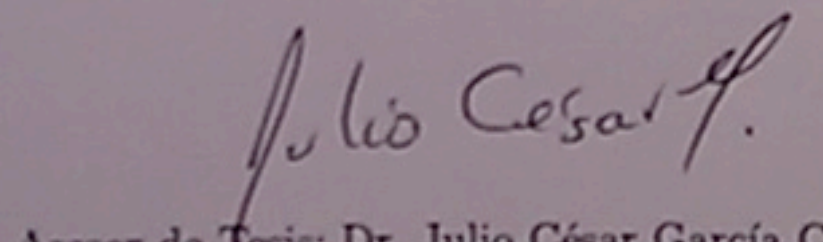

Asesor de Tesis: Dr. Julio César García Corte

Junio 29, 2012. 